\title{
Internationalität an deutschen Hochschulen
}

HSI-Monitor Gesamtbericht 2019/2020

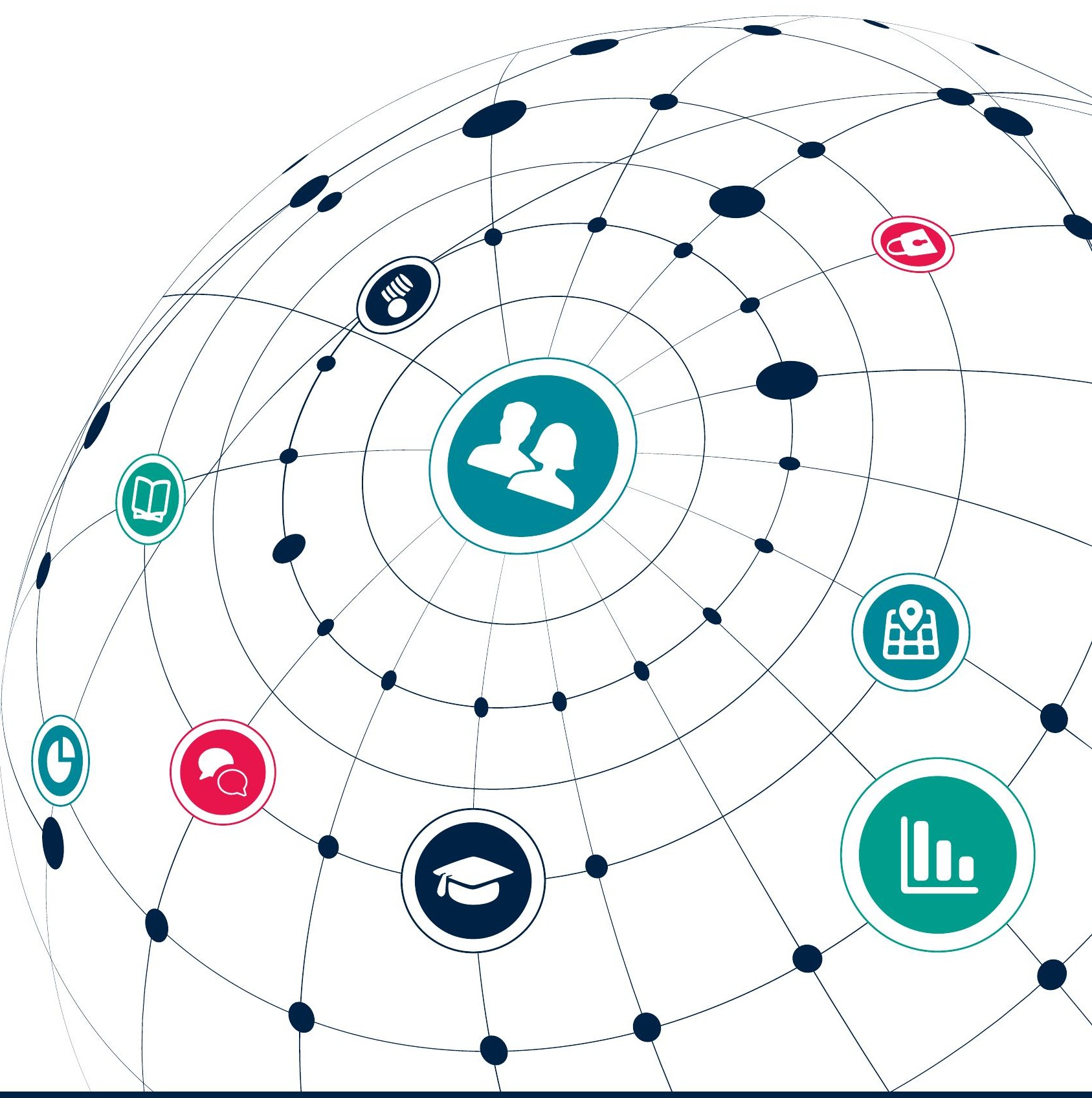





\section{Internationalität an deutschen Hochschulen}

HSI-Monitor Gesamtbericht 2019/2020

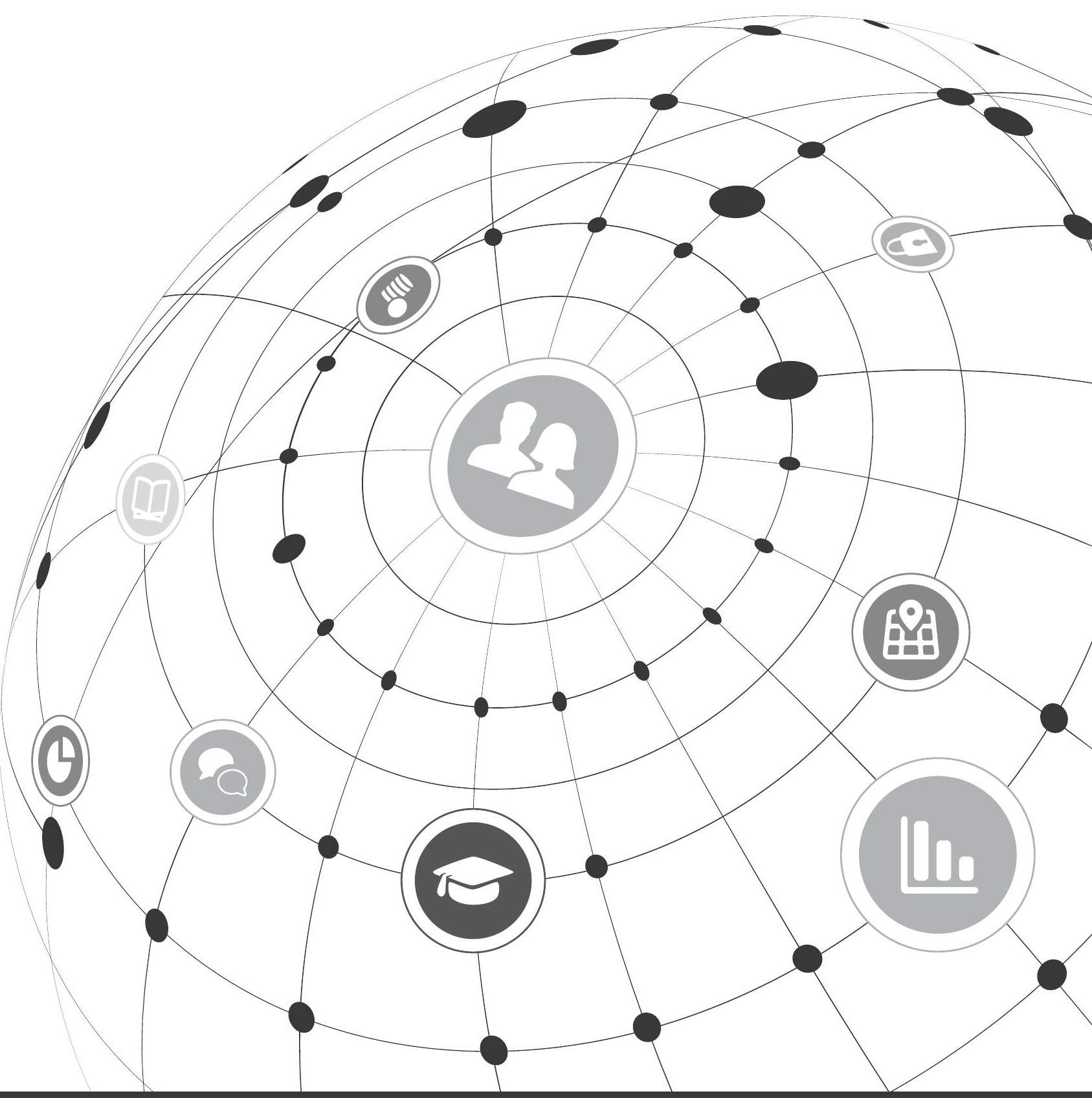




\section{Herausgeber}

Deutscher Akademischer

Austauschdienst

Kennedyallee 50, 53175 Bonn

www.daad.de

Referat S 15 - Forschung und Studien

\section{Alexander von Humboldt-Stiftung (AvH)}

Jean-Paul-Str. 12, 53173 Bonn

www.humboldt-foundation.de

Referat Evaluation und Statistik

\section{Deutsche Forschungsgemeinschaft (DFG)}

Kennedyallee 40, 53175 Bonn

www.dfg.de

Statistik und Reporting

\section{Hochschulrektorenkonferenz (HRK)}

Ahrstraße 39, 53175 Bonn

www.hrk.de

Arbeitsbereich Internationale

Angelegenheiten

\section{Autorinnen und Autoren}

Axel Karpenstein, DAAD

Diandra Dachtera, DAAD

\section{Datenaufbereitung}

Friedhelm Maiworm,

Gesellschaft für Empirische Studien (GES), Kassel

\section{Danksagung}

Wir bedanken uns bei Dr. Jan Kercher, DAAD,

für methodologische und konzeptionelle Beratung.

Diese Publikation wird aus Zuwendungen des

Bundesministeriumsfür Bildung und Forschung (BMBF)

an den DAAD finanziert.

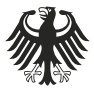

Bundesministerium

für Bildung

und Forschung

\section{Konzept}

Digitas Pixelpark GmbH, Köln

\section{Gestaltung und Satz}

Ditho Design GmbH, Köln

\section{Druck}

W. Kohlhammer Druckerei GmbH + Co. KG, Stuttgart

\section{Auflage}

Dezember $2020-1.000$

\section{Zitierhinweis}

DAAD / AvH / DFG / HRK (Hrsg.) (2020).

Internationalität an deutschen Hochschulen:

HSI-Monitor Gesamtbericht 2019/2020. Bonn: DAAD.

DOI: https://doi.org/10.46686/HSIMonitor.2020.gb

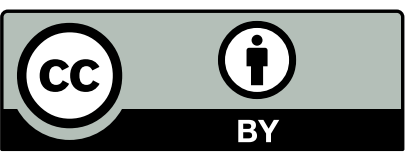

Dieses Material steht unter der Creative-CommonsLizenz Namensnennung 4.0 International (CC BY 4.0) Um eine Kopie dieser Lizenz zu sehen, besuchen Sie https://creativecommons.org/licenses/by/4.0/. 


\section{Vorwort}

Seit über zehn Jahren stellen wir als Serviceleistung Profildaten zur Internationalität der deutschen Hochschulen zusammen und bereiten sie in jährlichen Publikationen auf. Den Hochschulen können diese Daten als wichtige Werkzeuge für das Monitoring und die strategische Planung von Internationalisierungsmaßnahmen dienen; der interessierten Öffentlichkeit vermitteln sie Erkenntnisse zum Stand der Internationalisierung des deutschen Hochschulsystems.

Mit finanzieller Unterstützung des Bundesministeriums für Bildung und Forschung konnte im Zeitraum 2019/20 eine Digitalisierung der Profildaten, eine Erweiterung der Themenbereiche sowie ein Ausbau der Auswertungsmöglichkeiten erfolgen. Seit Mai 2020 steht das Online-Portal „HSI-Monitor - Profildaten zur Hochschulinternationalität" den Hochschulen, politischen Entscheidungsträgerinnen und -trägern sowie der interessierten Öffentlichkeit zur Verfügung. Der HSI-Monitor vereint die Vorteile eines digitalen Serviceangebots: Dazu gehören ein bequemer, standortunabhängiger Datenzugang, umfangreiche und flexible Auswertungsoptionen sowie die Möglichkeit, Daten und Diagramme schnell und einfach zur weiteren Verwendung herunterzuladen. Das neve Portal wird die Hochschulen daher noch besser unterstützen, aber gleichzeitig auch neue Nutzerinnen und Nutzer ansprechen.

Die Tradition eines gedruckten Jahresberichts möchten wir auch mit dem neuen HSI-Monitor fortsetzen, allerdings in zeitgemäßer Form. Der aktuelle Bericht 2019/2020 fasst weiterhin die aktuellen Entwicklungen in den Teilbereichen der Hochschulinternationalität zusammen und erlaubt eine differenzierte Betrachtung in Abhängigkeit von der Art und Größe der Hochschule. Damit liefert er wertvolle Bezugsgrößen für das Benchmarking. Eine umfassende Verlinkung des elektro- nischen Dokuments ermöglicht den direkten Zugang zu vertiefenden Texten und den Originaldaten und den Diagrammen im Portal.

Der diesjährige Bericht enthält neue, wichtige Themenbereiche wie zum Beispiel zusätzliche Daten zur Internationalität der Forschung, zur Geschlechterverteilung in der Internationalisierung, zu Partnerländern und -regionen sowie zu Fächergruppen. So wird ein noch umfassenderes Bild vom aktuellen Stand der Internationalität vermittelt.

Wir danken den beteiligten Hochschulen für ihre Mitwirkung beim Ausbau des Projekts.

Ebenso danken wir dem Bundesministerium für Bildung und Forschung für die Förderung unserer gemeinsamen Initiative.

Prof. Dr. Joybrato Mukherjee Präsident des Deutschen Akademischen Austauschdienstes

Prof. Dr. Peter-André Alt Präsident der Hochschulrektorenkonferenz

Prof. Dr. Hans-Christian Pape Präsident der Alexander von Humboldt-Stiftung

Prof. Dr. Katja Becker

Präsidentin der Deutschen Forschungsgemeinschaft

Bonn, im Dezember 2020 


\section{Inhalt}

\section{1}

Internationale Studierende
1.1 Grunddaten: Studierende
1.2 Studierende nach Abschlussart
1.3 Grunddaten: Absolventinnen und Absolventen

1.4 Absolventinnen und Absolventen nach Abschlussart

\section{3}

\section{Internationales Personal}

3.1 Personalgruppen im Überblick

3.2 Wissenschaftliches und künstlerisches Personal an Hochschulen

3.3 Professorinnen und Professoren

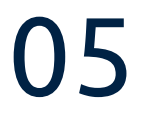

International ausgerichtete Studiengänge

5.1 Internationale und englischsprachige Studiengänge insgesamt

5.2 Internationale und englischsprachige Studiengänge nach Abschluss

5.3 Doppelabschlussstudiengänge

\section{2}

12 Internationale Promovierende

132.1 Promovierende

\section{4}

28 Erasmus-Mobilität

4.1 Studierende

4.2 Praktikantinnen und Praktikanten

4.3 Hochschulpersonal: Lehrkräfte

4.3 Hochschulpersonal:

Nicht-wissenschaftliches Personal

\section{6}

Internationale

42 Hochschulkooperationen

48

6.1 Grunddaten

51

6.2 Verteilung der Hochschulkooperationen nach Ländern und Regionen 


\section{7}

Internationale Publikationen

7.1 Grunddaten

7.2 Verteilung der internationalen

Ko-Publikationen nach Fächergruppe

und Partnerland

\section{9}

Forschungsförderung der

Alexander von Humboldt-Stiftung

9.1 Grunddaten

9.2 Verteilung der Aufenthalte von AvH-Geförderten nach Fächergruppen

9.3 Verteilung der Aufenthalte von AvH-Geförderten nach Herkunftsland

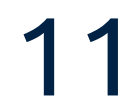

Drittmittel der Europäischen Union

\subsection{Grunddaten}

11.2 Fördermittel aus dem EU-Forschungsprogramm Horizont 2020

\section{8}

54 Förderungen des DAAD 60

568.1 Individualförderung

8.2 Geförderte im Rahmen des Programmes „PROMOS - Förderung der Mobilität von Studierenden und Promovierenden deutscher Hochschulen"

8.3 Förderung für Projekte und Programme

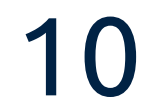

DFG-Projekte an Hochschulen

74

68

69

10.1 Grunddaten

10.2 Verteilung der DFG-Projekte an Hochschulen nach Fächergruppe

10.3 Verteilung der DFG-Projekte an Hochschulen nach Partnerland 
anmerkungen zur Benutzung des Berichts 


\section{Anmerkungen zur Nutzung des Berichts}

Der jährliche Gesamtbericht des HSI-Monitors fasst die wichtigsten Entwicklungen der Hochschulinternationalität geordnet nach Themenbereichen zusammen. Der Schwerpunkt liegt auf der Präsentation der zentralen Ergebnisse, gleichzeitig wird auf zusätzliche Erläuterungen und weiterführende Texte im Online-Portal verlinkt. Links sind durch die farbliche Hervorhebung und zwei spitze Klammern >> leicht erkennbar.

Links, die mit Lesen Sie mehr eingeleitet werden, führen Sie zu ausführlicheren Texten im HSI-Monitor.

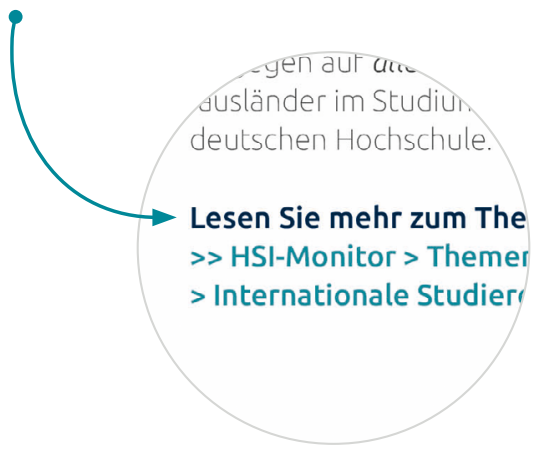

Auch die Diagramme und Tabellen sind durchgehend mit Verweisen auf das Online-Portal ausgestattet, durch Anklicken des Links gelangen Sie direkt zum jeweiligen Diagramm bzw. zur Tabelle. Dort können Sie Zahlen nachsehen oder das Diagramm Ihren Bedürfnissen entsprechend anpassen.

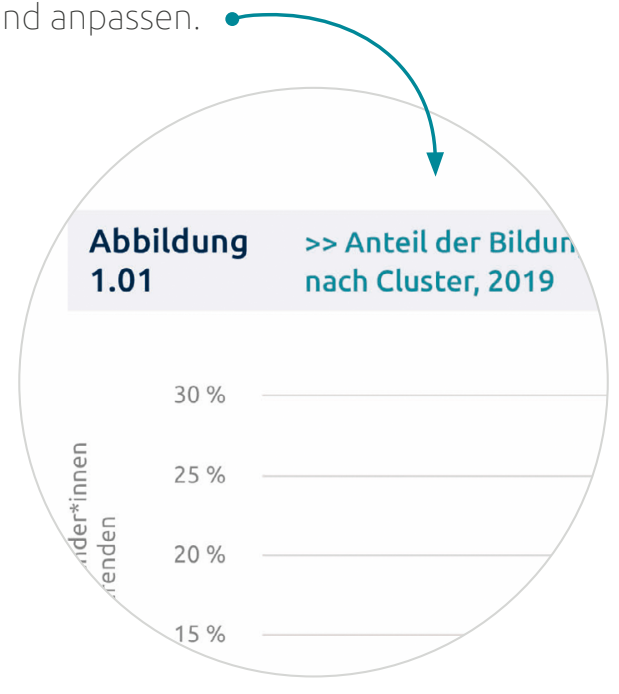

Mit dem Ausdruck Im Detail: sind Diagrammlinks gekennzeichnet, die zur Illustration der Analyseergebnisse herangezogen werden können. Mit Siehe auch: wird auf verwandte Diagramme verwiesen.

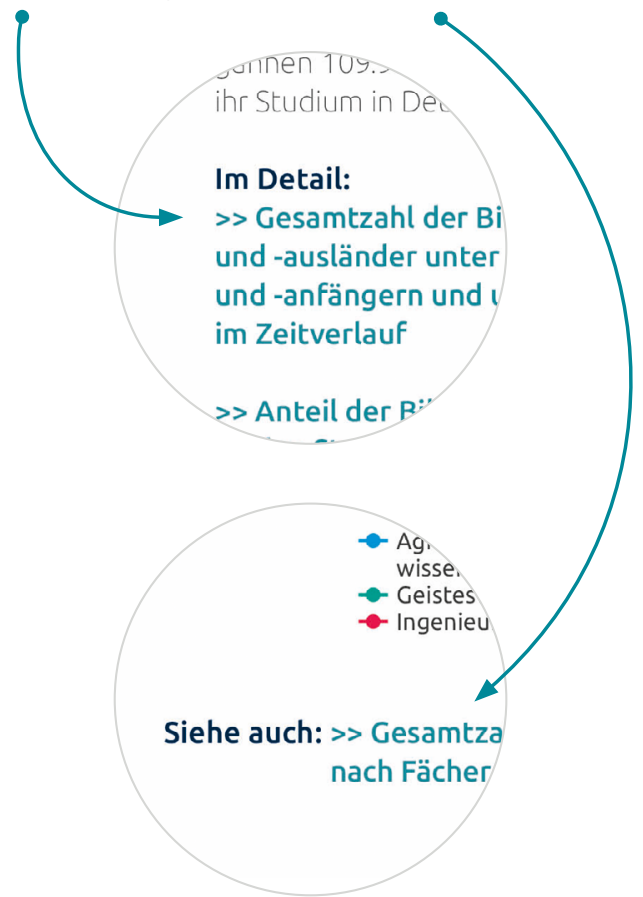

Sie haben auf einen Link geklickt, aber das Diagramm im Online-Portal ähnelt nicht dem im Bericht abgebildeten? Dies kann vorkommen, da die Daten im HSI-Monitor regelmäßig aktualisiert werden. Höchstwahrscheinlich werden im Online-Diagramm Daten zu einem weiteren Jahr angezeigt. Darum finden Sie im HSI-Monitor unter jedem Diagramm die Versionen. Sie können sich jederzeit frühere Versionen des Diagramms über die Versionierungsleiste anzeigen lassen. Informationen zu den Datenquellen finden Sie ebenfalls unter den Diagrammen in den Metadaten.

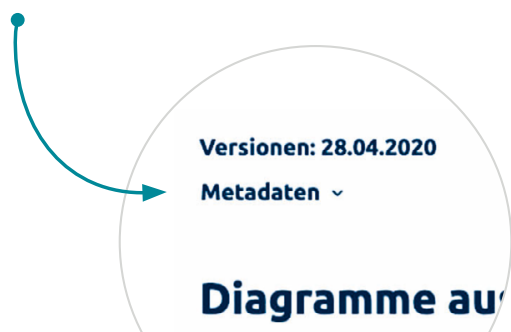


Die im Gesamtbericht enthaltenen Diagramme wurden alle über das Online-Portal HSI-Monitor generiert.

Da im gedruckten Bericht die Flexibilität der Online-

Darstellung verloren geht, empfehlen wir Ihnen,

die Verlinkung zum Online-Portal besonders dann zu

nutzen, wenn die Lesbarkeit der Diagramme zum Bei-

spiel durch nahe beieinanderliegende Werte erschwert wird. In den Online-Diagrammen können durch Klicken in der Legende einzelne Zahlenreihen ausgeblendet werden, die Skalierung des Diagramms wird dann entsprechend angepasst.
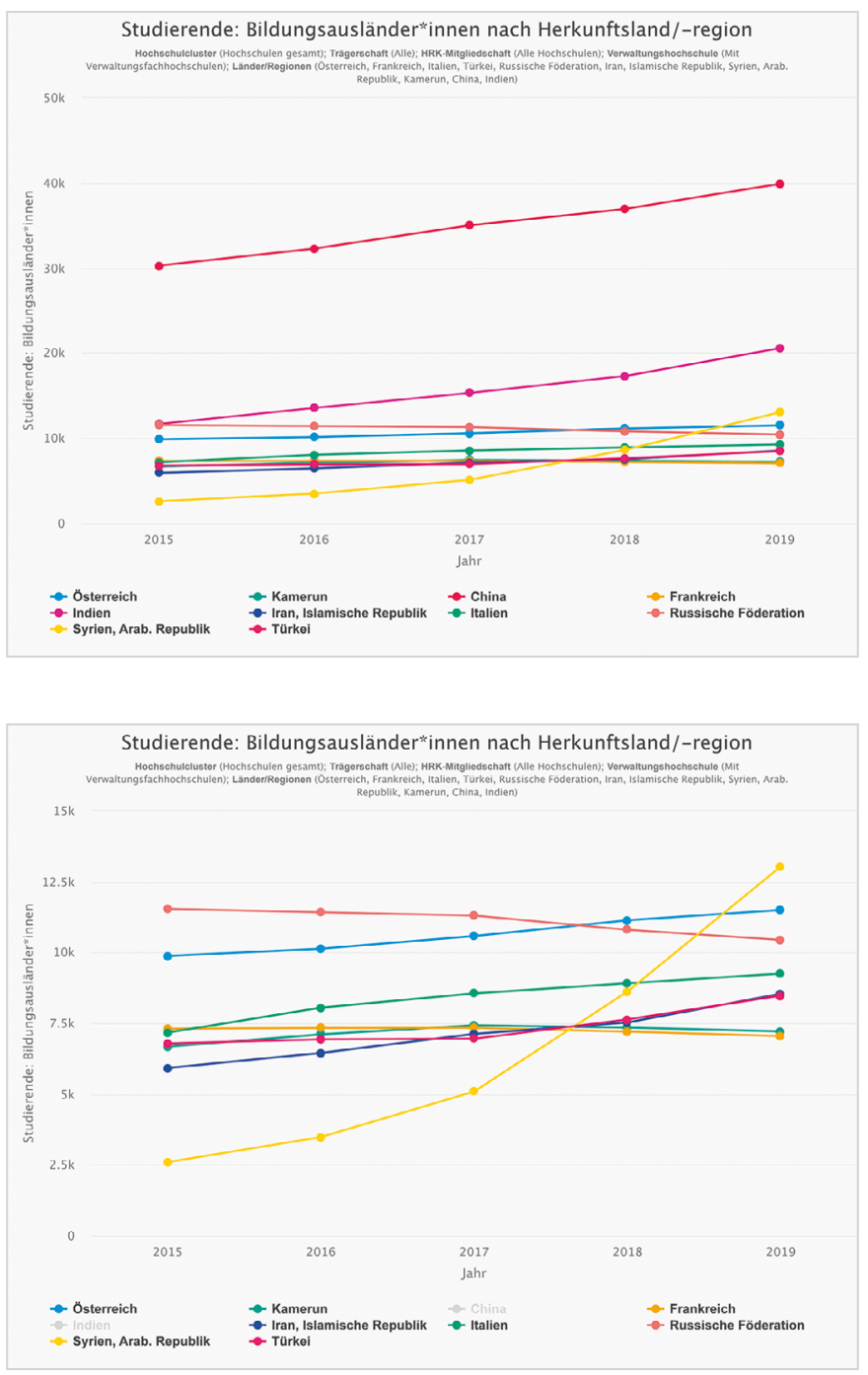

Im obigen Beispiel wurden die Zahlenreihen für China und Indien durch Klicken auf die entsprechenden Bezeichnungen in der Legende ausgeblendet. Die Skalierung der y-Achse passt sich der verringerten Spannweite an, so dass die übrigen Zahlenreihen entzerrt dargestellt werden. 
Informationen zur Methodik der Datenerhebung,

den Datenquellen sowie zu den Hochschulclustern

finden Sie im HSI-Monitor. Mehr lesen: >> Methodik

Fachhochschulen (FHs) und Hochschulen für Angewandte Wissenschaften (HAWs) werden, der Praxis des Statistischen Bundesamtes folgend, im HSI-Monitor und im Bericht zusammenfassend als „Fachhochschulen" bezeichnet. Erklärungen der im Bericht verwendeten Begriffe und Abkürzungen können Sie im HSI-Monitor nachlesen:

\section{Mehr lesen: >> Glossar / Abkürzungsverzeichnis.}

Dieser Bericht ist als PDF Download mit den integrierten Links online verfügbar: Download: >> HSI-Monitor > Berichte \& Analysen > HSI-Monitor: Gesamtberichte oder nutzen Sie den QR-Code im Impressum.

Im Gegensatz zu den bisherigen Profildatenberichten, bei denen im Titel auf das aktuellste Jahr der Datenlieferung Bezug genommen wurde, wird der Gesamtbericht des HSI-Monitors zukünftig nach Publikationsjahr benannt. In seiner Funktion als Brücke zwischen den Profildatenberichten und den Gesamtberichten des HSI-Monitors erscheint der vorliegende Bericht als "Cesamtbericht 2019/2020".
Im Bericht wird die ausgeschriebene Gendervariante (z.B. „Bildungsausländerinnen und -ausländer") genutzt. Im Online-Portal „HSI-Monitor" werden jedoch zukünftig neben den Geschlechtskategorien „männlich”, „weiblich“ ebenfalls Daten zum dritten Geschlecht "divers" ausgegeben. Deshalb wird in der Programmierung des Portals das Gendersternchen verwendet. Die Diagramme im Bericht werden direkt aus dem Portal generiert und übernehmen dann gegebenenfalls das Gendersternchen bei der Achsenbezeichnung. 


\section{园 Internationale Studierende}

- Im Jahr 2019 studierten über 300.000 Bildungsausländerinnen und -ausländer an deutschen Hochschulen. Fast 45.000 machten im gleichen Jahr einen Abschluss in Deutschland.

- Inzwischen sind etwa ebenso viele Bildungsausländerinnen und -ausländer in einem Bachelor- wie in einem Master-Studiengang eingeschrieben. Unter den Absolventinnen und Absolventen ist die Zahl der Bildungsausländerinnen und -ausländer mit Master-Abschluss mehr als doppelt so hoch wie die mit Bachelor-Abschluss.

- Die Ingenieurswissenschaften haben seit 2010 zunehmend an Bedeutung gewonnen, 2019 gingen fast $40 \%$ der Bildungsausländerinnen und -ausländer einem Studium in dieser Fächergruppe nach, 37\% machten darin einen Abschluss.

- Die wichtigsten Herkunftsländer für Bildungsausländerinnen und -ausländer im Studium waren 2019 China, Indien und Syrien. Bei den Absolventinnen und Absolventen eines Studiums in Deutschland kamen die meisten Bildungsausländerinnen und -ausländer aus China, Indien und Russland. 


\section{Internationale Studierende}

Differenzierte Daten zu ausländischen Studienanfängerinnen und -anfängern, Studierenden sowie Absolventinnen und Absolventen werden jährlich vom Statistischen Bundesamt (Destatis) erhoben und veröffentlicht. Die Daten erlauben vielfältige Auswertungen, unter anderem nach angestrebtem bzw. erreichten Abschluss, Geschlecht, Fächergruppe oder Herkunftsland. Die Auswertungen nach Abschluss beschränken sich im vorliegenden Bericht auf das Bachelor- und Master-Studium, Daten zu anderen
Abschlüssen sowie zu Studierenden ohne Abschlussabsicht sind im HSI-Monitor einsehbar. Die Gesamtauswertungen (Kapitel 1.1 und 1.3) beziehen sich hingegen auf alle Bildungsausländerinnen und -ausländer im Studium bzw. mit Abschluss an einer deutschen Hochschule.

\section{Lesen Sie mehr zum Thema unter: \\ >> HSI-Monitor > Themen \\ $>$ Internationale Studierende}

\subsection{Grunddaten: Studierende}

Sowohl die absolute Zahl der Bildungsausländerinnen und -ausländer in einem Studium in Deutschland wie auch ihr Anteil an der Gesamtzahl der Studierenden ist von 2018 auf 2019 weiter gestiegen. 2019 studierten 302.157 von ihnen an deutschen Hochschulen und damit 7,1\% mehr als im Vorjahr. Im gleichen Jahr begannen 109.955 Bildungsausländerinnen und -ausländer ihr Studium in Deutschland, 4,8\% mehr als im Jahr $2018 .{ }^{1}$

\section{Im Detail:}

>> Gesamtzahl der Bildungsausländerinnen und -ausländer unter den Studienanfängerinnen und -anfängern und unter den Studierenden im Zeitverlauf

>> Anteil der Bildungsausländerinnen und -ausländer an den Studienanfängerinnen und -anfängern und an den Studierenden im Zeitverlauf

Den höchsten Anteil von Bildungsausländerinnen und -ausländern an allen Studierenden hatten im Jahr 2019 mit 27,7\% die Kunst- und Musikhochschulen, gefolgt von den kleinen und den großen Universitäten (13,2\% bzw. 11,9\%). Ein ähnliches Bild bietet sich bei den Studienanfängerinnen und -anfängern, auch hier wiesen die Kunst- und Musikhochschulen mit 48,8\% den höchsten Anteil an Bildungsausländerinnen und -ausländern auf, vor den kleinen und den großen Universitäten (30,9\% bzw. 26,7\%). siehe Abb. 1.01, S. 14
39,6\% der Bildungsausländerinnen und -ausländer (119.666 Personen) studierten 2019 in einem Fach der Ingenieurswissenschaften und 24,9\% (75.261) in den Rechts-, Wirtschafts- und Sozialwissenschaften. Bei den Geisteswissenschaften und Mathematik/Naturwissenschaften waren die Zahlen erheblich niedriger und unterschieden sich nur geringfügig (10,9\% / 33.081 bzw. 10,8\% / 32.709). Der Anteil der Ingenieurswissenschaften ist zwischen 2016 und 2019 um 3,4\% gestiegen, der Anteil der Geisteswissenschaften und der Rechts-, Wirtschafts- und Sozialwissenschaften leicht gefallen. Abb. 1.02, S. 14

Die meisten Bildungsausländerinnen und -ausländer, die 2019 an deutschen Hochschulen eingeschrieben waren, kamen aus China (39.880 bzw. 13,2\% aller Bildungsausländerinnen und -ausländer im Studium), gefolgt von Indien (20.562 bzw. 6,8\%) und Syrien (13.032 bzw. 4,3\%). In den letzten Jahren hat vor allem der Anteil der indischen und syrischen Studierenden zugenommen. Für Studierende aus Russland war eine geringe, aber kontinuierliche Abnahme zu beobachten.

- Abb. 1.03, S. 15

1 Aufgrund unterschiedlicher Bezugszeiträume ist die Zahl der Studienanfängerinnen und -anfänger eines Jahrgangs keine direkte Untergruppe der Gesamtzah der eingeschriebenen Studierenden. Als Studienanfängerinnen und -anfänger werden alle Studierenden gezählt, die im Studienjahr (Sommer- und nachfolgendes Wintersemester) im ersten Hochschul- oder Fachsemester eingeschrieben waren; die Zahl der Studierenden gibt dagegen den Stand der immatrikulierten Studierenden im Wintersemester wieder. 


\section{Abbildung > Anteil der Bildungsausländerinnen und -ausländer an allen Studierenden}

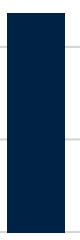

它

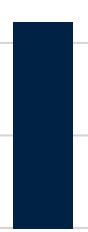

高

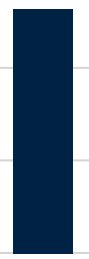

㐫

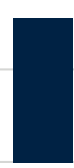

沜

2019

- Anteil der Bildungsausländer*innen an allen Studierenden

Siehe auch: >> Anteil der Bildungsausländerinnen und -ausländer an allen Studienanfängerinnen und -anfängern nach Cluster, 2019

Abbildung >> Verteilung der Bildungsausländerinnen und -ausländer 1.02 im Studium nach Fächergruppe, 2016-2019

$50 \%$

$40 \%$

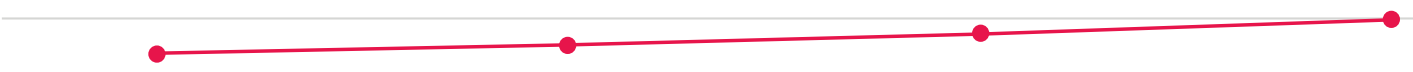

$30 \%$

$20 \%$

$10 \%$

$0 \%$

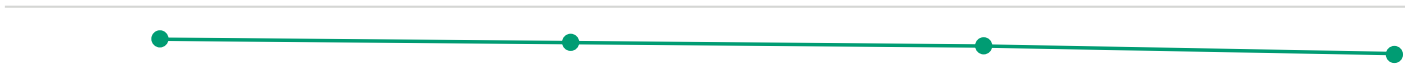

Agrar-, Forst- und Ernährungswissenschaften, Veterinärmedizin

- Geisteswissenschaften

- Ingenieurwissenschaften
- Kunst, Kunstwissenschaft

- Mathematik, Naturwissenschaften

- Humanmedizin/Gesundheitswissenschaften

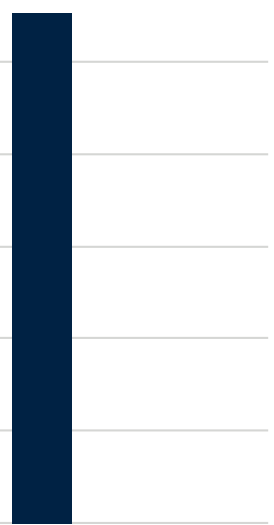

$\sum_{\underline{I}}^{I}$ 
Abbildung > Gesamtzahl der Bildungsausländerinnen und -ausländer im Studium 1.03 nach Herkunftsland, 2015-2019

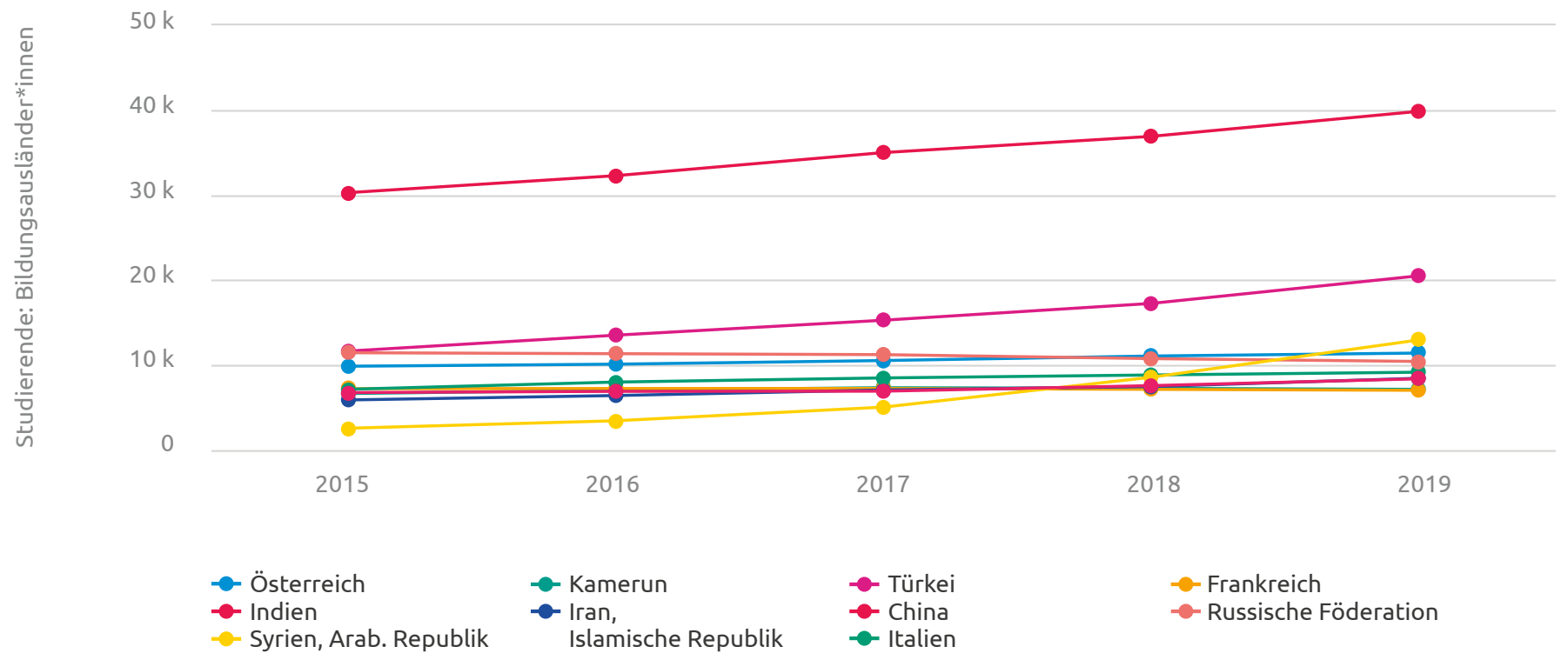

Siehe auch: >> Verteilung der Bildungsausländerinnen und -ausländer im Studium nach Herkunftsland, 2015-2019

Abbildung > Gesamtzahl der Bildungsausländerinnen und -ausländer 1.04 im Bachelor- und im Master-Studium im Zeitverlauf

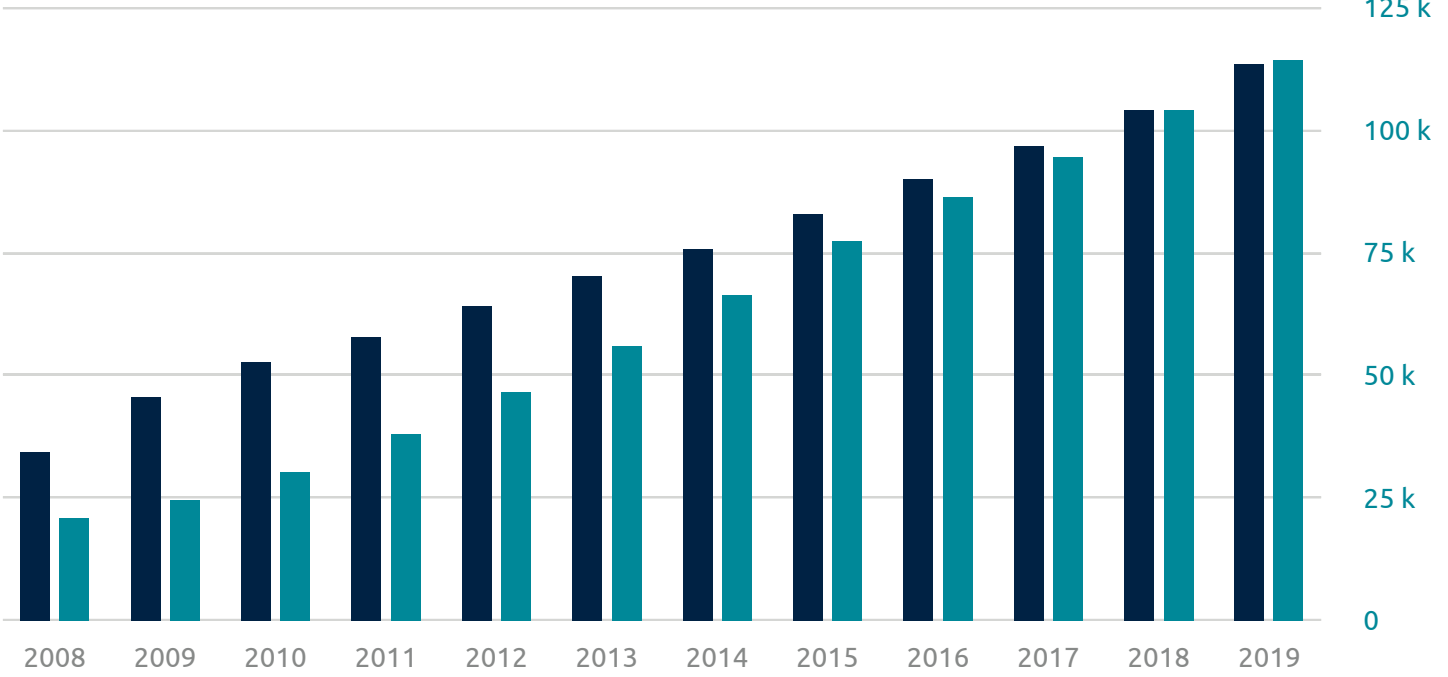

- (A) Studierende im Bachelor-Studium: Bildungsausländer*innen

- (B) Studierende im Master-Studium: Bildungsausländer*innen 


\subsection{Studierende nach Abschlussart}

Sowohl die Zahlen der Bildungsausländerinnen und -ausländer im Bachelor-Studium als auch im Master-Studium sind weiter gestiegen. Seit 2010 liegt die Zuwachsrate im Master-Studium über der im Bachelor-Studium; 2019 strebten in Deutschland erstmalig mehr Bildungsausländerinnen und -ausländer einen Master-als einen Bachelor-Abschluss an (114.641 gegenüber 113.730).

Abb. 1.04, S. 15

Kunst- und Musikhochschulen wiesen 2019 sowohl im Bachelor- als auch im Master-Studium sehr viel höhere Anteile an Bildungsausländerinnen und -ausländern auf als die anderen Hochschulcluster (Bachelor: 20,7\%, Master: 42,9\%). Im Bachelor-Studium lag dieser Anteil durchschnittlich bei den Fachhochschulen mit 6,1\% geringfügig über dem Wert bei den Universitäten (5,8\%); im Master-Studium hingegen lagen die Universitäten mit einem Anteil von 18,1\% vor den Fachhochschulen (15,6\%).

\section{Im Detail:}

>> Anteil der Bildungsausländerinnen und -ausländer an allen Studierenden im Bachelor-Studium nach Cluster, 2019

>> Anteil der Bildungsausländerinnen und -ausländer an allen Studierenden im Master-Studium nach Cluster, 2019

Für die populärsten Fächergruppen ist zu beobachten, dass sie von den Bildungsausländerinnen und -ausländern im Bachelor und im Master-Studium ähnlich stark nachgefragt werden. So werden die Ingenieurwissenschaften von 47,6\% der Bildungsausländerinnen und -ausländer im Bachelorstudium gewähtt und von
45,9\% derer im Masterstudium. Vergleichbare Anteile sind auch in den Rechts-, Wirtschafts- und Sozialwissenschaften (Bachelor: 27,7\%, Master: 24,9\%) und in der Fächergruppe Mathematik/Naturwissenschaften (Bachelor: 8,3\%, Master: 9,8\%) zu beobachten.

\section{Im Detail:}

>> Verteilung der Bildungsausländerinnen und -ausländer im Bachelor-Studium nach Fächergruppe, 2016-2019

\section{>> Verteilung der Bildungsausländerinnen und -ausländer im Master-Studium nach Fächer- gruppe, 2016-2019}

Die meisten Bildungsausländerinnen und -ausländer im Bachelor- und auch im Master-Studium kamen im Jahr 2019 aus China (12.137 bzw. 10,7\% aller Bildungsausländerinnen und -ausländer im BachelorStudium; 18.659 bzw. 16,3\% im Master-Studium). Im Bachelor-Studium folgten auf dem zweiten und dritten Platz Syrien (7.858 bzw. 6,9\% der Bildungsausländerinnen und -ausländer im Bachelor-Studium) und Österreich (6.158 bzw. 5,4\%). Abb. 1.05 Zwischen 2015 und 2019 ist der Anteil der syrischen Studierenden um 6,1 Prozentpunkte gestiegen. Einen leichten Anstieg um 1,3 Prozentpunkte bzw. 1,1 Prozentpunkte gab es bei Studierenden aus Tunesien und aus Vietnam.

Im Master-Studium lagen Indien mit 16.947 bzw. 14,8\% der Bildungsausländerinnen und -ausländer und Iran mit 4.335 bzw. 3,8\% an zweiter und dritter Stelle. Abb. 1.06 Der Anteil der indischen Studierenden ist zwischen 2015 und 2019 um 3 Prozentpunkte gestiegen, der Anteil der syrischen Studierenden um 1,5 Prozentpunkte.

\section{Weitere Auswertungsmöglichkeiten}

Der HSI-Monitor enthält über die Grunddaten und die Daten nach Abschlussart hinaus weitere Informationen zu Studierenden und Absolventinnen und Absolventen, zum Beispiel zur Art des Studiums (Erststudium, konsekutives MasterStudium, etc.) und zur Mobilität von Bildungsausländerinnen und -ausländern (studienbezogene vs. abschlussbezogene Mobilität, auch nach Fächergruppe und Herkunftsland/-region).

Mehr hierzu finden Sie auf den Themenseiten >> Studierende nach Art des Studiums und $\gg$ A Absolventinnen und Absolventen nach Art des Studiums sowie in der >> Übersicht der Diagramme. 
Abbildung > Gesamtzahl der Bildungsausländerinnen und -ausländer im Bachelor-Studium 1.05 nach Herkunftsland, 2015-2019
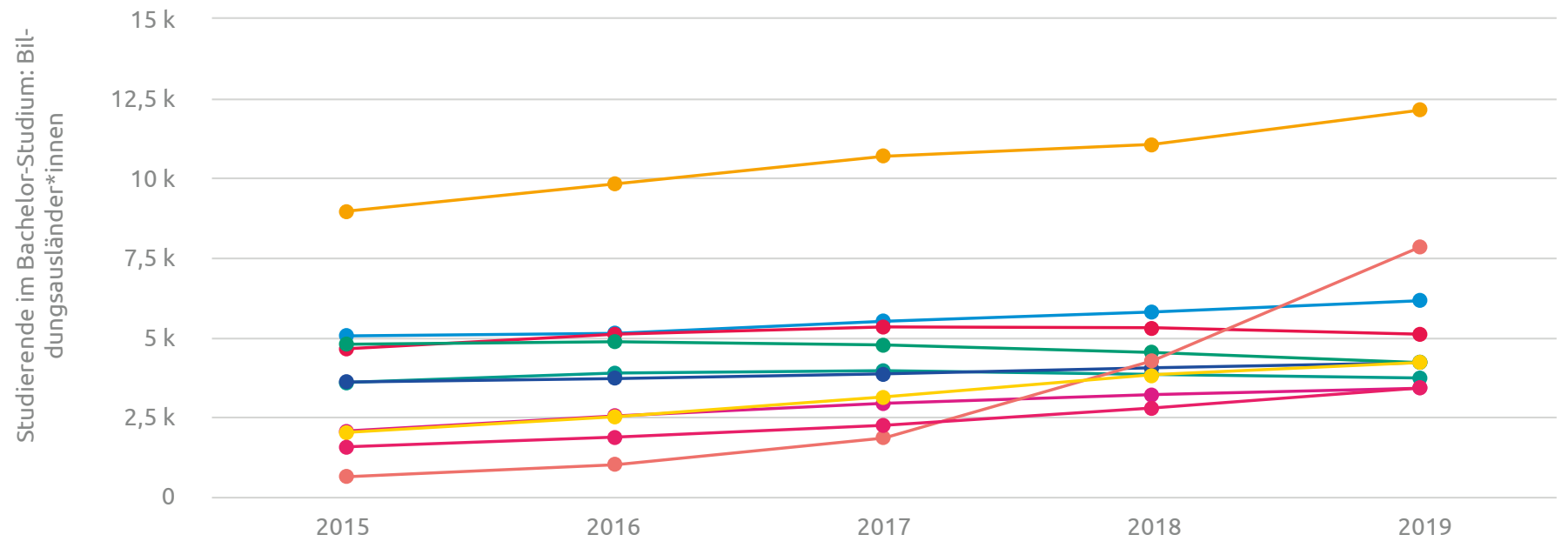

- Österreich
- Indonesien
- Tunesien

- Bulgarien

Vietnam

Siehe auch: >> Verteilung der Bildungsausländerinnen und -ausländer im Bachelor-Studium nach Herkunftsland, 2015-2019

Abbildung >> Gesamtzahl der Bildungsausländerinnen und -ausländer im Master-Studium 1.06 nach Herkunftsland, 2015-2019

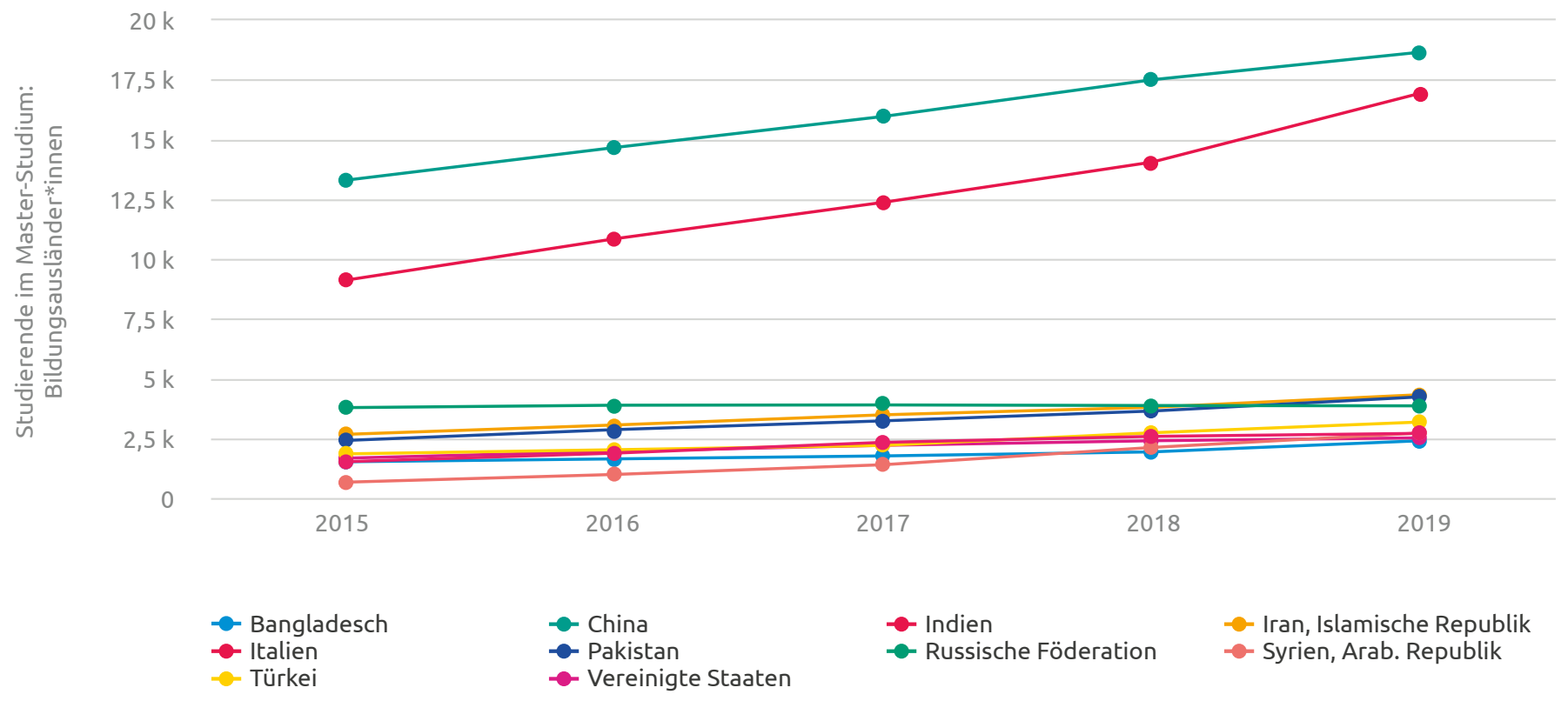

Siehe auch: >> Verteilung der Bildungsausländerinnen und -ausländer im Master-Studium nach Herkunftsland, 2015-2019 


\subsection{Grunddaten: Absolventinnen und Absolventen}

Die Zahl der Bildungsausländerinnen und -ausländer, die ein Studium an einer deutschen Hochschule absolviert haben, ist weiter gestiegen und lag 2019 bei 43.981; dies bedeutet eine Zunahme um 5,4\% im Vergleich zum Vorjahr.

\section{Im Detail:}

>> Gesamtzahl der Bildungsausländerinnen und -ausländer unter den Absolventinnen und Absolventen im Zeitverlauf

\section{> Anteil der Bildungsausländerinnen} und -ausländer an den Absolventinnen und Absolventen im Zeitverlauf

Gemessen an der Gesamtzahl der Absolventinnen und Absolventen war der Anteil der Bildungsausländerinnen und -ausländer im Jahr 2019 bei den Kunst- und Musikhochschulen mit 30,8\% am höchsten. Universitäten kamen im Durchschnitt auf einen Anteil von 9,1\%, wobei die kleinen Universitäten vor den großen und den mittelgroßen lagen. Fachhochschulen wiesen durchschnittlich einen Anteil von 6\% auf. Der Wert war bei den mittelgroßen Fachhochschulen höher als bei den großen und den kleinen. Abb. 1.07

Die meisten Bildungsausländerinnen und -ausländer schlossen 2019 ein Studium der Ingenieurswissenschaften ab (16.269 bzw. 37,0\% aller Bildungsausländerinnen und -ausländer), gefolgt von den Rechts-, Wirtschafts- und Sozialwissenschaften (12.351 bzw. 28,1\%) und der Fächergruppe Mathematik/ Naturwissenschaften (4.914 bzw. 11,2\%).

\section{Im Detail:}

>> Gesamtzahl der Bildungsausländerinnen und -ausländer unter den Absolventinnen und Absolventen nach Fächergruppe, 2016-2019

>> Verteilung der Bildungsausländerinnen und -ausländer unter den Absolventinnen und Absolventen nach Fächergruppe, 2016-2019

\section{Abbildung >> Anteil der Bildungsausländerinnen und -ausländer an allen Absolventinnen und Absolventen nach Cluster, 2019}

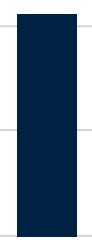

㐫

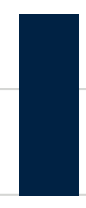

$\cdot \frac{\dot{\bar{E}}}{\bar{c}}$

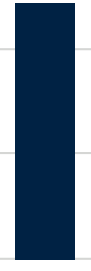

立

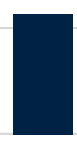

它

2019

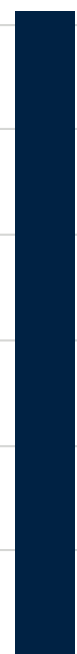

$\underline{\sum}$

- Anteil der Bildungsausländer*innen an allen Absolvent*innen 
Von den Bildungsausländerinnen und -ausländern, die 2019 ein Studium in Deutschland abschlossen, kamen 7.096 (16,1\%) aus China, 3.590 (8,2\%) aus Indien und 1.992 (4,5\%) aus Russland. Bei den zehn wichtigsten Herkunftsländern hat zwischen 2015 und 2019 besonders der Anteil der Absolventinnen und Absolventen aus Indien zugenommen.
Im Detail:

>> Gesamtzahl der Bildungsausländerinnen und -ausländer unter den Absolventinnen und Absolventen nach Herkunftsland, 2015-2019

>> Verteilung der Bildungsausländerinnen und -ausländer unter den Absolventinnen und Absolventen nach Herkunftsland, 2015-2019

\subsection{Absolventinnen und Absolventen nach Abschlussart}

Mit 24.768 gegenüber 11.701 Absolventinnen und Absolventen erwarben 2019 mehr als doppelt so viele Bildungsausländerinnen und -ausländer einen Masterwie einen Bachelor-Abschluss in Deutschland. Die Zahl der Absolventinnen und Absolventen nimmt bei beiden Abschlüssen zu. Verglichen mit dem Vorjahr betrug der Anstieg im Jahr 2019 für das Bachelor-Studium 5,8\% und für das Master-Studium 8,8\%. Abb. 1.08

Abbildung > Gesamtzahl der Bildungsausländerinnen und -ausländer unter den Absolventinnen 1.08 und Absolventen eines Bachelor- und eines Master-Studiums im Zeitverlauf
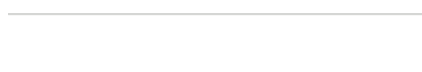

$20 \mathrm{k}$

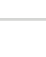

$15 k$

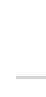

$10 \mathrm{k}$
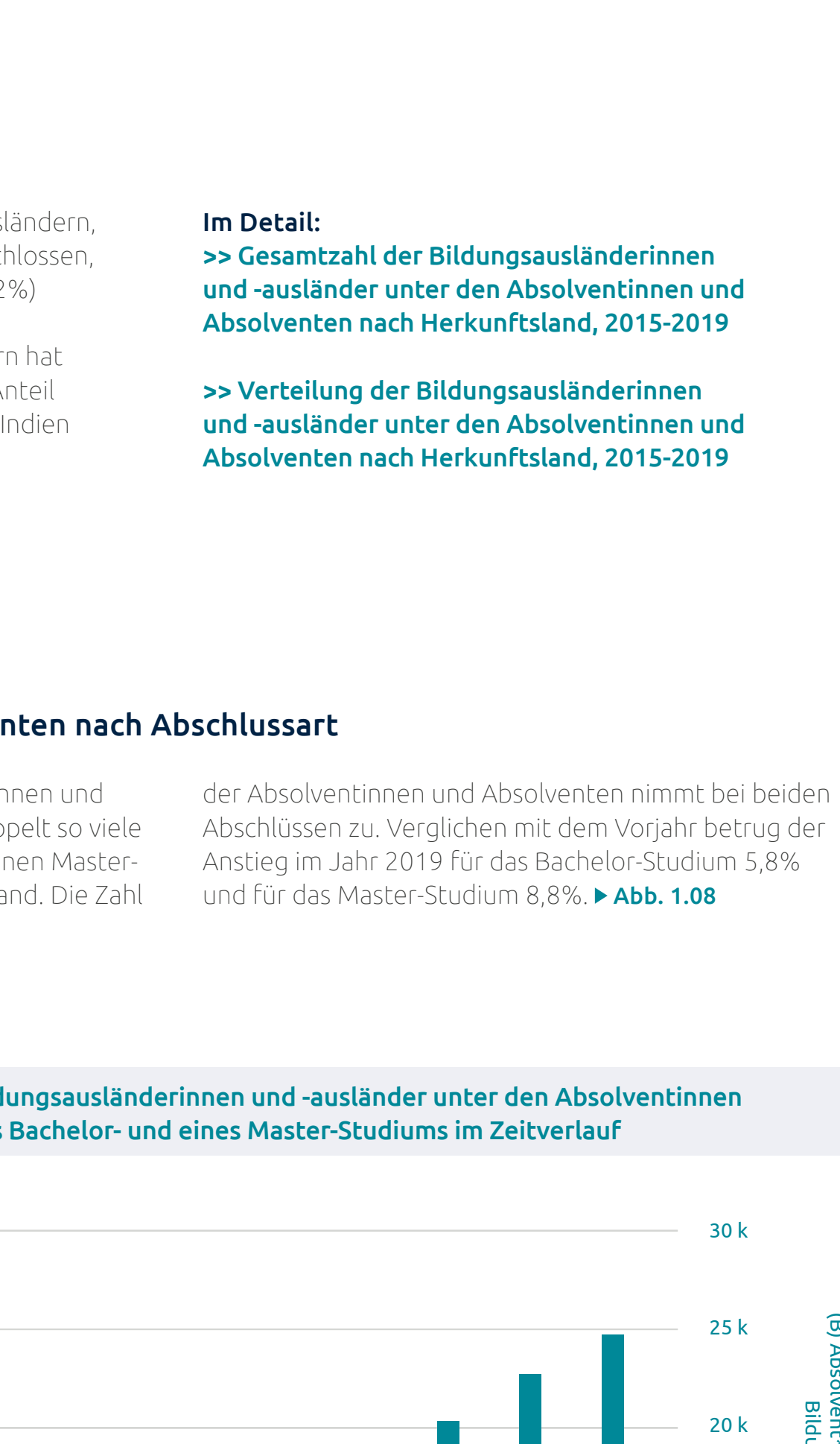

- (A) Absolvent*innen eines Bachelor-Studiums: Bildungsausländer*innen

- (B) Absolvent*innen eines Master-Studiums: Bildungsausländer*innen 
Abbildung > Gesamtzahl der Absolventinnen und Absolventen eines Bachelor-Studiums:

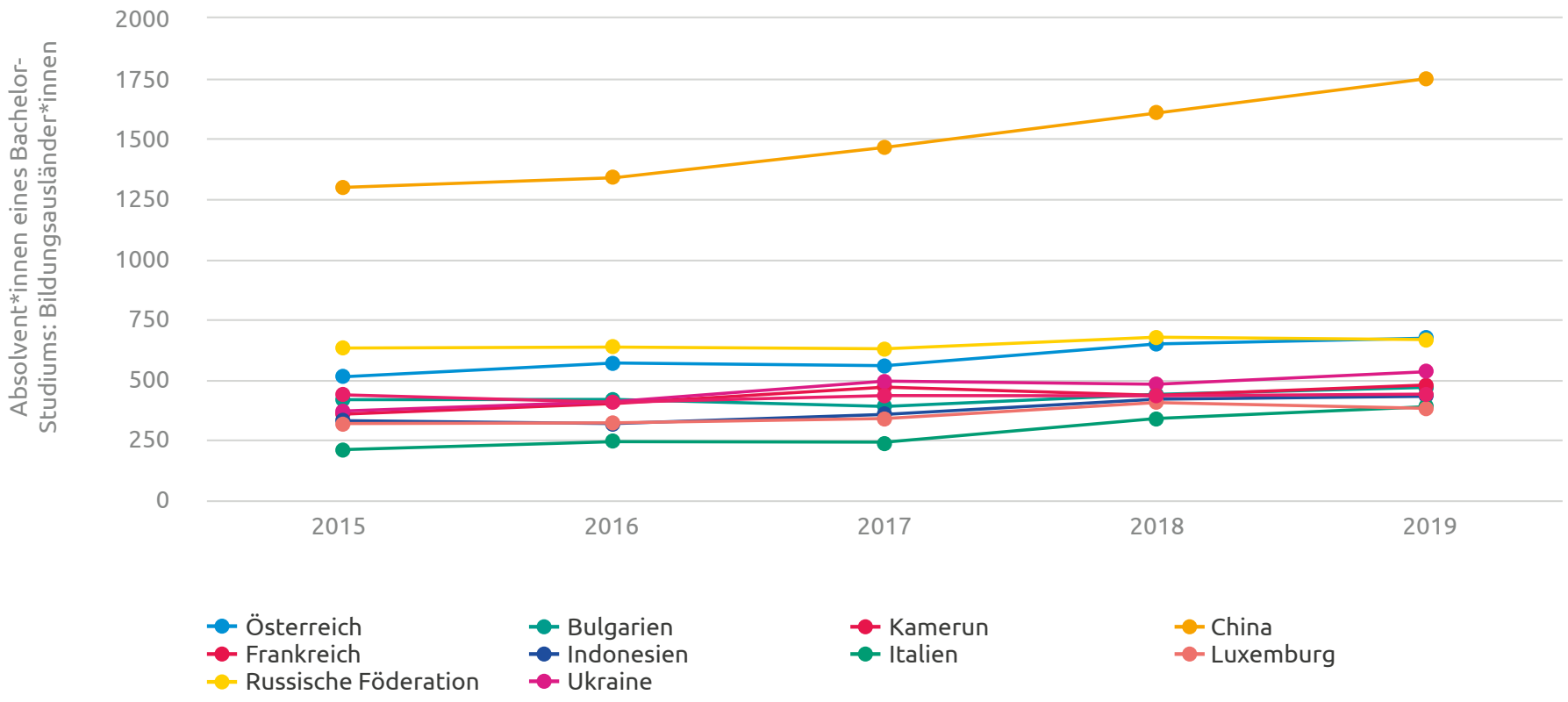

Siehe auch: >> Verteilung der Bildungsausländerinnen und -ausländer unter den Absolventinnen und Absolventen eines Bachelor-Studiums nach Herkunftsland, 2015-2019

Abbildung > Gesamtzahl der Absolventinnen und Absolventen eines Master-Studium: 1.10 Bildungsausländerinnen und -ausländer nach Herkunftsland, 2015-2019

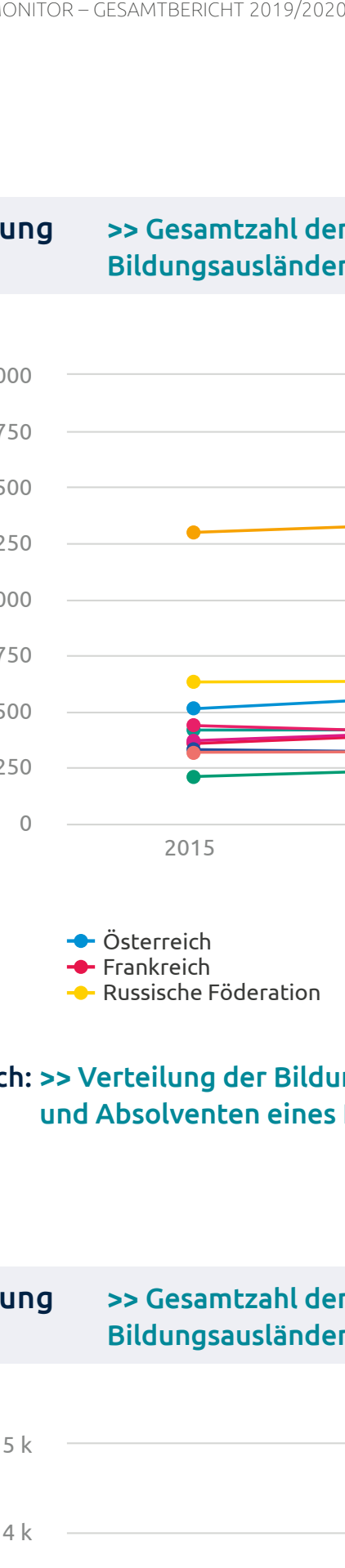

$3 k$

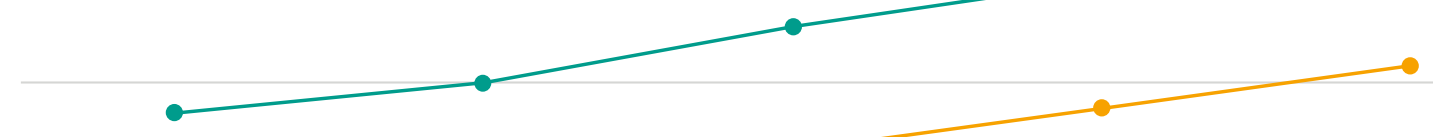

$2 \mathrm{k}$

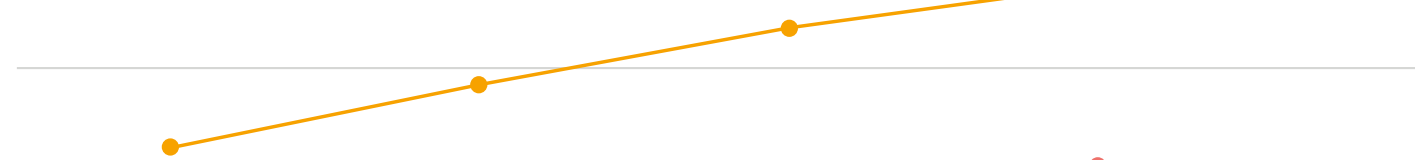

$1 \mathrm{k}$

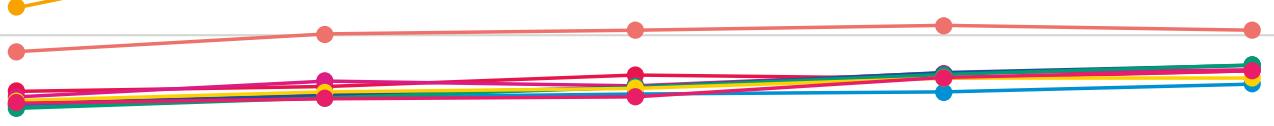

0

2015

2016

2017

2018

2019

- Österreich
- Iran, Islamische Republik
- Ukraine

- China

- Italien

- Vereinigte Staaten

- Frankreich
- Pakistan

- Indien

Siehe auch: >> Verteilung der Bildungsausländerinnen und -ausländer unter den Absolventinnen und Absolventen eines Master-Studiums nach Herkunftsland, 2015-2019 
Bei den Absolventinnen und Absolventen sowohl eines Bachelor- als auch eines Master-Studiums sind die Anteile der Bildungsausländerinnen und -ausländer mit 18,9\% (Bachelor-Abschluss) bzw. 44,2\% (MasterAbschluss) bei den Kunst- und Musikhochschulen am höchsten. An den Universitäten liegt dieser Wert bei durchschnittlich 4,4\% (Bachelor-Abschluss) bzw. 15,9\% (Master-Abschluss), die Fachhochschulen kommen auf durchschnittlich 4,1\% (BachelorAbschluss) bzw. 12,5\% (Master-Abschluss).

\section{Im Detail:}

\section{>> Anteil der Bildungsausländerinnen} und -ausländer an allen Absolventinnen und Absolventen eines Bachelor-Studiums nach Cluster, 2019

\section{>> Anteil der Bildungsausländerinnen und -ausländer an allen Absolventinnen und Absolventen eines Master-Studiums nach Cluster, 2019}

Fast drei Viertel der Bildungsausländerinnen und -ausländer im Bachelor-Studium graduierten 2019 in den Ingenieurswissenschaften (37,7\%) oder den Rechts-, Wirtschafts- und Sozialwissenschaften (35,2\%). Es folgten die Geisteswissenschaften (9,6\% der graduierten Bildungsausländerinnen und -ausländer) sowie die Fächergruppe Kunst-/Kunstwissenschaft $(8,2 \%)$. Einen Master-Abschluss in den Ingenieurswissenschaften machten im gleichen Jahr $42,3 \%$ der Bildungsausländerinnen und -ausländer, während 28,7\% einen Master-Abschluss in den Rechts-, Wirtschaftsund Sozialwissenschaften erhielten. Die Geisteswissenschaften waren mit 9,3\% am drittstärksten, die Fächergruppe Mathematik/Naturwissenschaften mit 7,9\% am viertstärksten vertreten.
Im Detail:

>> Verteilung der Bildungsausländerinnen und -ausländer unter den Absolventinnen und Absolventen im Bachelor-Studium nach Fächergruppe, 2016-2019

\section{>> Verteilung der Bildungsausländerinnen und -ausländer unter den Absolventinnen und Absolventen im Master-Studium nach Fächergruppe, 2016-2019}

Die meisten Bildungsausländerinnen und -ausländer, die im Jahr 2019 in Deutschland einen Bachelor-Abschluss erwarben, kamen aus China (1.749 bzw. 14,9\% aller Bildungsausländerinnen und -ausländer mit BachelorAbschluss), gefolgt von Österreich (673 bzw. 5,8\%) und Russland (667 bzw. 5,8\%). In den letzten fünf Jahren hat unter den zehn wichtigsten Herkunftsländern besonders der Anteil der Bachelor-Absolventinnen und -Absolventen aus China und Italien zugenommen.

- Abb. 1.09

Bei den Bildungsausländerinnen und -ausländern, die einen Master-Abschluss erwarben, kam fast ein Drittel aus China (4.313 bzw. 17,4\% aller Bildungsausländerinnen und -ausländer mit Master-Abschluss) und Indien (3.152 bzw. 12,7\%); drittwichtigstes Herkunftsland war Russland (1.045 bzw. 4,2\%). Unter den zehn wichtigsten Herkunftsländern hat zwischen 2015 und 2019 besonders Indien an relativer Bedeutung gewonnen. Abb. $\mathbf{1 . 1 0}$ 


\section{( Internationale Promovierende}

- Bildungsausländerinnen und -ausländer stellten im Jahr 2019 knapp ein Viertel der eingeschriebenen Promovierenden in Deutschland und knapp $18 \%$ der abgeschlossenen Promotionsverfahren.

- Die meisten Bildungsausländerinnen und -ausländer strebten 2019 eine Promotion in Mathematik/Naturwissenschaften (35\%), Ingenieurswissenschaften $(21 \%)$ oder Geisteswissenschaften (15\%) an.

- Der größte Teil der abgeschlossenen Promotionsvorhaben entfiel im gleichen Jahr auf die Fächergruppen Mathematik/Naturwissenschaften (43\%), Ingenieurswissenschaften (20\%) und Humanmedizin/ Gesundheitswissenschaften (12\%).

- China und Indien waren im Jahr 2019 die wichtigsten Herkunftsländer von promovierenden Bildungsausländerinnen und -ausländern, Iran lag auf Platz drei. Auch bei den abgeschlossenen Promotionen führen China und Indien vor Italien an dritter Stelle. 


\section{Internationale Promovierende}

Ausländische Promovierende bereichern die deutsche Forschungslandschaft und helfen Lücken zu schließen, wo der Bedarf an Fachkräften besonders hoch ist. Der Anteil der ausländischen Promovierenden ist einerseits eine wichtige Kennzahl für den Grad der Internationalisierung des deutschen Wissenschaftssystems, andererseits kann er auch als ein Indikator für die internationale Attraktivität des Forschungsstandorts Deutschland angesehen werden.

Die Daten zu Promovierenden und abgeschlossenen Promotionsverfahren basieren auf der Studierendenstatistik bzw. der Prüfungsstatistik des Statistischen
Bundesamtes (Destatis). Obgleich in Deutschland die Möglichkeiten zur Anfertigung einer Promotion an einer Fachhochschule im Rahmen von kooperativen Promotionen oder hochschulinternen oder hochschulübergreifenden Promotionszentren erweitert worden sind, sind die absoluten Zahlen der darauf fußenden Promotionen noch gering. Der Themenbereich „Internationale Promovierende" ist daher auf Universitäten und Technische Universitäten ausgerichtet.

\section{Lesen Sie mehr zum Thema unter: \\ >> HSI-Monitor > Themen > Internationale Promovierende}

\subsection{Promovierende}

Von den 27.341 Bildungsausländerinnen und -ausländern, die 2019 in Deutschland in einem Promotionsstudium eingeschrieben waren, studierten 68,0\% an einer großen Universität, 25,3\% an einer mittelgroßen Universität und 5,7\% an einer kleinen Universität. Der Anteil der Bildungsausländerinnen und -ausländer an allen Promovierenden ist seit 2011 beständig gestiegen und lag 2019 bei 24,9\%.

\section{Im Detail:}

>> Anteil der Bildungsausländerinnen und -ausländer an allen Studierenden im Promotionsstudium im Zeitverlauf
Die Unterschiede zwischen den Hochschulclustern in Bezug auf den Anteil der Bildungsausländerinnen und -ausländer an allen Promovierenden sind vergleichsweise klein. Den höchsten Anteil weisen die mittelgroßen Universitäten auf (2019: 26,0\%), bei den kleinen Universitäten ist er am geringsten (2019: 23,8\%). Bei den großen und den mittelgroßen Universitäten ist in den letzten Jahren ein leichter Anstieg zu beobachten. A Abb. 2.01

\section{Abbildung

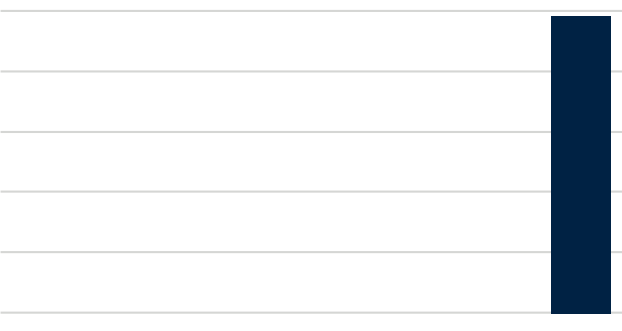

Uni gr.

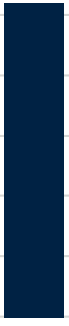

Uni mi.

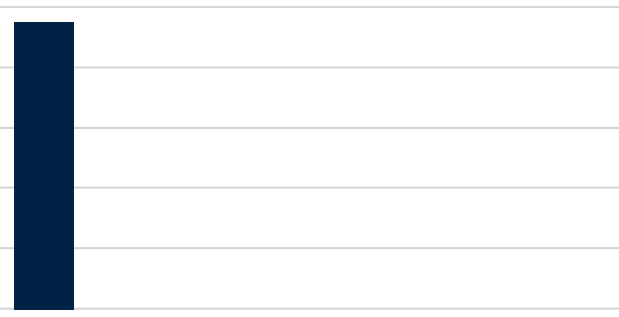

Uni kl. 
9.673 bzw. 35,4\% der Bildungsausländerinnen und -ausländer im Promotionsstudium promovierten 2019 in der Fächergruppe Mathematik/Naturwissenschaften. An zweiter Stelle lagen die Ingenieurswissenschaften mit 5.672 bzw. 20,8\% der Bildungsausländerinnen und -ausländer, an dritter die Geisteswissenschaften mit 4.220 bzw. 15,4\%. Die deutlichste Veränderung in Bezug auf die Verteilung zwischen den Fächergruppen zeigen die Humanmedizin/Gesundheitswissenschaften, ihr Anteil ist zwischen 2016 und 2019 von $7,5 \%$ auf $9,7 \%$ gestiegen. ${ }^{2}$

\section{Im Detail:}

> Gesamtzahl der Bildungsausländerinnen und -ausländer im Promotionsstudium nach Fächergruppe, 2016-2019

\author{
>> Verteilung der Bildungsausländerinnen \\ und -ausländer im Promotionsstudium \\ nach Fächergruppe, 2016-2019
}

\begin{abstract}
4.759 bzw. 17,4\% der Bildungsausländerinnen und -ausländer im Promotionsstudium kamen im Jahr 2019 aus China. Zweitwichtigstes Herkunftsland war Indien mit 1.842 bzw. 6,7\% der Bildungsausländerinnen und -ausländer, drittwichtigstes Herkunftsland war Iran mit 1.747 bzw. 6,4\%. A Abb. 2.02
\end{abstract}

Zwischen 2015 und 2019 waren die Anteile der zehn wichtigsten Herkunftsländer weitgehend stabil, nur der Anteil der chinesischen Promovierenden ist etwas stärker um 1,7 Prozentpunkte gestiegen.

Betrachtet man die Unterschiede zwischen den Hochschulclustern, war der Anteil der Bildungsausländerinnen und -ausländer im Jahr 2019 mit 19,1\% bei den kleinen Universitäten am höchsten, vor den großen Universitäten (18,3\%) und den mittelgroßen Universitäten (15,8\%). Abb. 2.03

\section{Im Detail:}

>> Anteil der Bildungsausländerinnen und -ausländer an den abgeschlossenen Promotionsverfahren im Zeitverlauf 
Abbildung

2.02

>> Gesamtzahl der Bildungsausländerinnen und -ausländer im Promotionsstudium nach Herkunftsland, 2015-2019

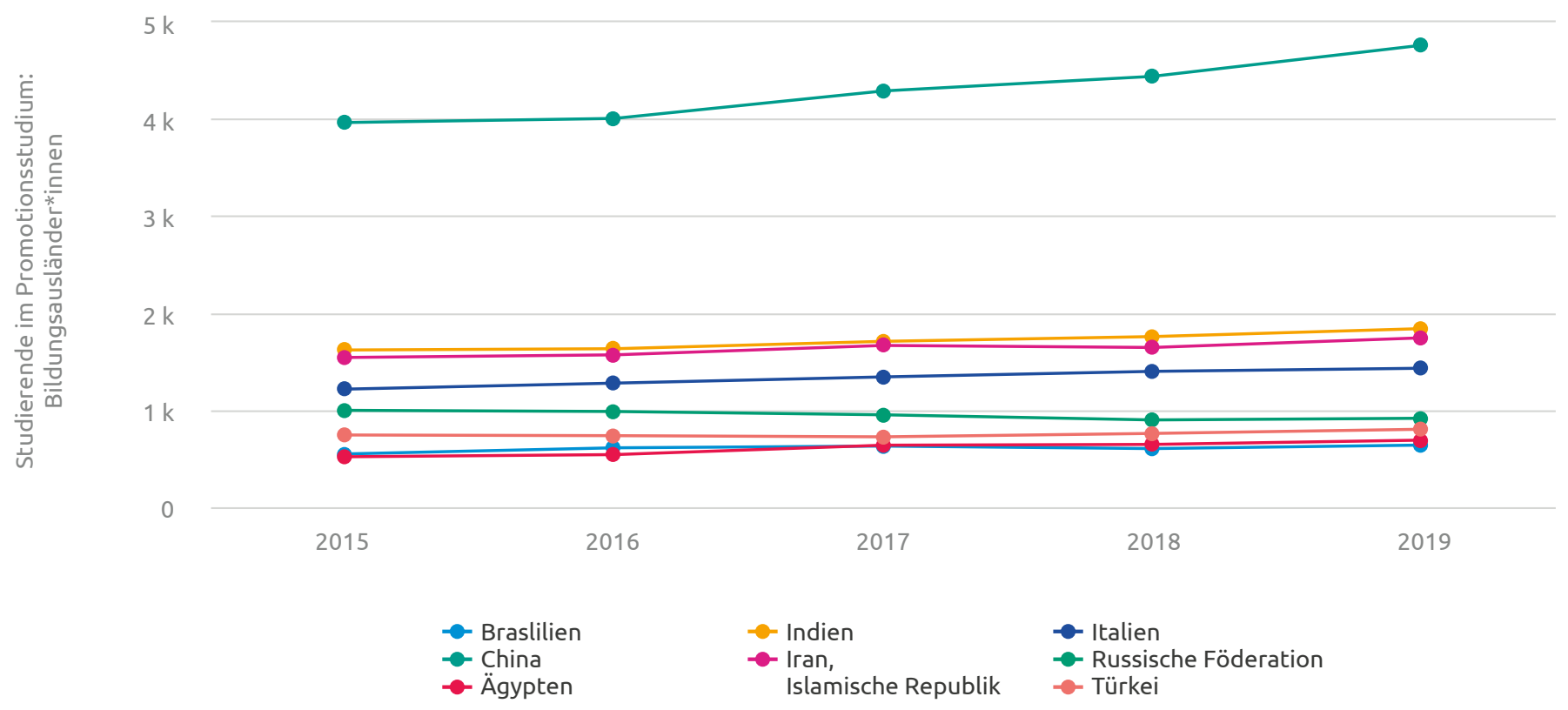

Siehe auch: >> Verteilung der Bildungsausländerinnen und -ausländer im Promotionsstudium nach Herkunftsland, 2015-2019

Abbildung >> Anteil der Bildungsausländerinnen und -ausländer an den Absolventinnen 2.03 und Absolventen eines Promotionsstudiums nach Cluster, 2019: Universitäten

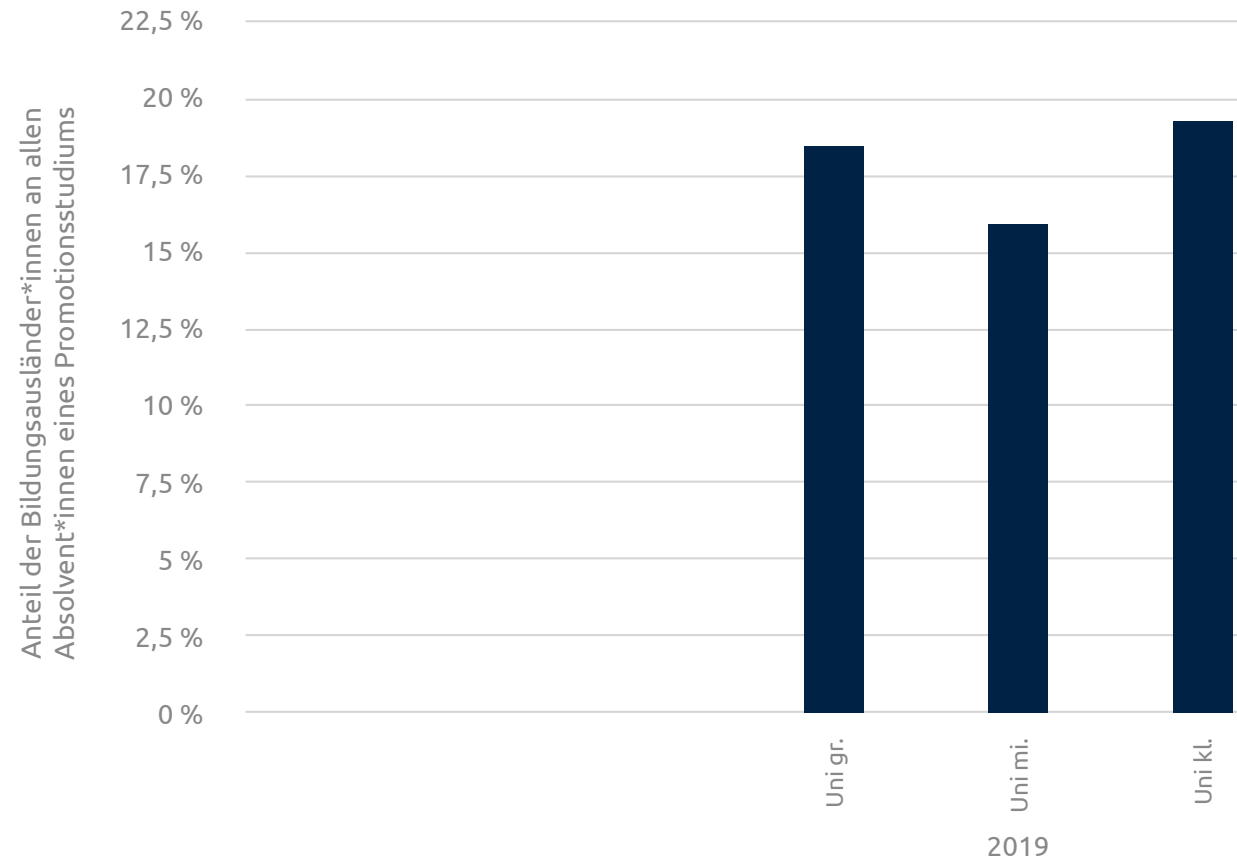

- Anteil der Bildungsausländer*innen an allen Absolvent*innen eines Promotionsstudiums 
Abbildung >> Verteilung der Bildungsausländerinnen und -ausländer mit Abschluss eines Promotionsstudiums nach Fächergruppe, 2019

\section{$50 \%$}

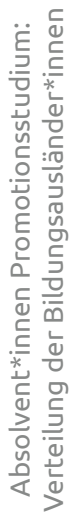

$40 \%$

$30 \%$

$20 \%$

$10 \%$

$0 \%$

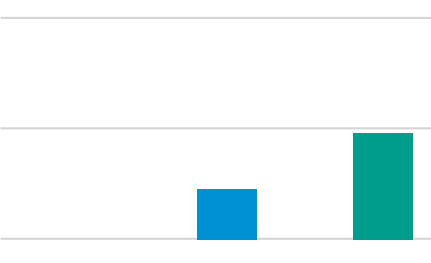

Agrar-, Forst- und Ernährungswissenschaften, Veterinärmedizin

- Geisteswissenschaften

Ingenieurwissenschaften
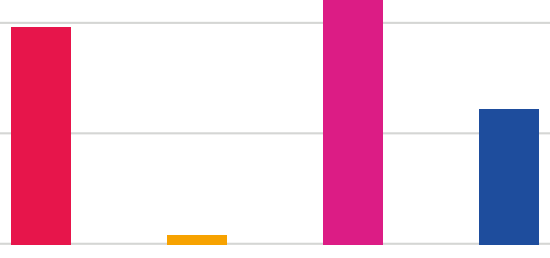

2019

Siehe auch: >> Gesamtzahl der Bildungsausländerinnen und -ausländer mit Abschluss eines Promotionsstudiums nach Fächergruppe, 2015-2019

Abbildung > Gesamtzahl der Bildungsausländerinnen und -ausländer mit Abschluss

2.05 eines Promotionsstudiums nach Herkunftsland, 2015-2019

- Kunst, Kunstwissenschaft

- Mathematik, Naturwissenschaften

- Humanmedizin/

Gesundheitswissenschaften
Rechts-, Wirtschaftsund Sozialwissenschaften $\rightarrow$ - Sport

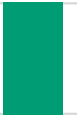

. 
Im Jahre 2019 schlossen 2.096 bzw. 42,8\% der

Bildungsausländerinnen und -ausländer ein Promotionsverfahren in Mathematik/Naturwissenschaften ab. Auf Platz zwei folgten die Ingenieurswissenschaften mit 963 bzw. 19,6\% der promovierten Bildungsausländerinnen und -ausländer und auf Platz drei die Fächergruppe Humanmedizin/Gesundheitswissenschaften mit 598 bzw. 12,2\% der promovierten Bildungsausländerinnen und -ausländer.

766 Bildungsausländerinnen und -ausländer bzw. 15,6\% aller Bildungsausländerinnen und -ausländer, die 2019 eine Promotion abschlossen, kamen aus China. Zweitwichtigstes Herkunftsland war Indien mit 357 bzw. 7,3\% der promovierten Bildungsausländerinnen und -ausländer, drittwichtigstes Land war Italien mit 332 bzw. 6,8\%. Abb. 2.05 In den letzten fünf Jahren haben sich die Anteile der zehn wichtigsten Herkunftsländer nur wenig verändert; einen stärkeren Anstieg gab es jedoch bei den Promotionen von Bildungsausländerinnen und -ausländern aus Italien und Spanien. 


\section{(2) Internationales Personal}

- Im Jahr 2019 arbeiteten knapp 50.000 wissenschaftliche und künstlerische Beschäftigte mit ausländischer Staatsbürgerschaft an deutschen Hochschulen, dies entsprach einem Anteil von $12,3 \%$ des gesamten wissenschaftlichen und künstlerischen Personals.

- Die größte Untergruppe bildeten mit ca. $60 \%$ die wissenschaftlichen und künstlerischen Mitarbeiterinnen und Mitarbeiter. Professorinnen und Professoren stellten knapp 7\% des wissenschaftlichen und künstlerischen Personals mit ausländischer Staatsbürgerschaft.

- Die wichtigsten Herkunftsländer beim ausländischen Personal insgesamt waren Italien, China und Indien mit Anteilen zwischen 6-7\%. Wichtigstes Herkunftsland in Bezug auf ausländische Professorinnen und Professoren war Österreich mit einem Anteil von 20\%; die Schweiz und Italien lagen mit zwischen 8-9\% auf den Plätzen 2 und 3. 


\section{Internationales Personal}

Eine Öffnung der Hochschulen für ausländisches Personal betrifft sowohl die Nachwuchswissenschaftlerinnen und -wissenschaftler, den akademischen Mittelbau, als auch die Hochschullehrerinnen und -lehrer. Die Personalstatistik des Statistischen Bundesamts (Destatis) hält die Staatsbürgerschaft der Hochschulangehörigen fest, wodurch der Anteil der Beschäftigten mit ausländischem Pass ermittelt werden kann. Bei der Verwendung dieser Kennzahl als Indikator für die Internationalität der Hoch-

\subsection{Personalgruppen im Überblick}

Die Personalstatistik von Destatis erlaubt umfassende Einzelbetrachtungen zu den verschiedenen Personalgruppen der Hochschulbeschäftigten. In der folgenden Tabelle sind die Beschäftigten mit ausländischer Staatsbürgerschaft sowie deren Anteil an den einzelnen Personalkategorien des haupt- und nebenberuflichen wissenschaftlichen und künstlerischen Personals im Jahr 2019 zusammengefasst. schulen ist zu beachten, dass eine ausländische Staatsbürgerschaft nicht zwangsläufig bedeutet, dass die betreffende Person im Ausland geboren und zur Schule gegangen ist. Eine Kategorie ähnlich den „Bildungsausländerinnen und -ausländern" der Studierendenstatistik existiert aktuell in der Personalstatistik nicht.

\section{Lesen Sie mehr zum Thema unter:}

>> HSI-Monitor > Themen > Internationales Personal
Im vorliegenden Bericht wird die Internationalität der Gesamtheit des wissenschaftlichen und künstlerischen Personals sowie die der Professorinnen und Professoren im Detail analysiert.

\section{Personalkategorie}

Hauptberufliches wissenschaftliches und künstlerisches Personal

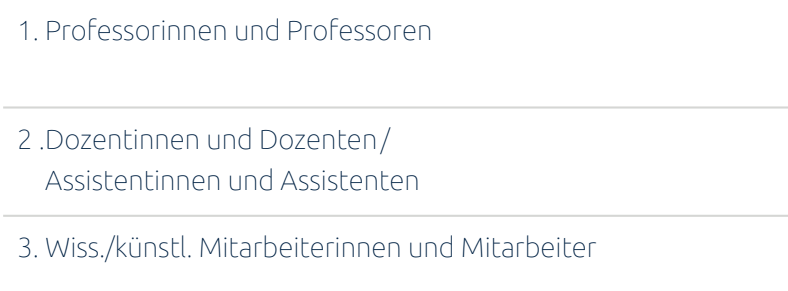

4. Lehrkräfte mit besonderen Aufgaben

$3,1 \%$

\section{Diagramme im HSI-Monitor}

Anteil am ausländischen Personal (2019)
> Gesamtzahl im Zeitverlauf

> Anteil an Gesamt im Zeitverlauf

$0,9 \%$

$59,8 \%$
> Gesamtzahl im Zeitverlauf

> Anteil an Gesamt im Zeitverlauf

> Gesamtzahl im Zeitverlauf

>> Anteil an Gesamt im Zeitverlauf

>> Gesamtzahl im Zeitverlauf

> Anteil an Gesamt im Zeitverlauf

Nebenberufliches wissenschaftliches und künstlerisches Personal

\author{
5. Gastprofessorinnen und -professoren / \\ Emeriti und Emeritae
}

\begin{abstract}
6. Lehrbeauftragte
\end{abstract}
$0,3 \%$

$17,4 \%$
>> Gesamtzahl im Zeitverlauf

> Anteil an Gesamt im Zeitverlauf

>> Gesamtzahl im Zeitverlauf

> Anteil an Gesamt im Zeitverlauf 


\subsection{Wissenschaftliches und künstlerisches Personal an Hochschulen}

In den letzten fünf Jahren hat die Gesamtzahl des wissenschaftlichen und künstlerischen Personals mit ausländischer Staatsbürgerschaft um 20,9\% von 41.010 auf 49.601 zugenommen. Besonders stark fältt dies bei den mittelgroßen Fachhochschulen auf, hier ist die Zahl des Personals mit ausländischer Staatsbürgerschaft um mehr als 50\% von 1.649 auf 2.550 gestiegen.

\section{Im Detail:}

>> Wiss./künstl. Personal mit ausländischer Staatsbürgerschaft im Zeitverlauf

Gemessen an der Gesamtzahl des wissenschaftlichen und künstlerischen Personals nahmen die Beschäftigten mit ausländischer Staatsbürgerschaft im Jahr 2019 einen Anteil von 12,3\% ein. Seit dem Jahr 2010, als der Anteil noch bei 9,9\% lag, ist er kontinuierlich gestiegen. Den höchsten Anteil des Personals mit ausländischer Staatsbürgerschaft wiesen im Jahr 2019 die Kunst- und Musikhochschulen auf (18,7\%); bei den Universitäten lag er bei durchschnittlich 14,2\%, bei den Fachhochschulen bei durchschnittlich 5,9\%. Abb. 3.01
Die meisten ausländischen Beschäftigten waren 2019 in der Fächergruppe Mathematik/Naturwissenschaften $(21,2 \%)$ tätig, vor den Ingenieurswissenschaften (19,1\%) und der Fächergruppe Humanmedizin/Gesundheitswissenschaften (18,7\%). Der Anteil der letztgenannten ist zwischen 2016 und 2019 leicht gestiegen, während derjenige der Geisteswissenschaften im gleichen Zeitraum leicht gesunken ist. ${ }^{3}$ - Abb. $\mathbf{3 . 0 2}$

Die wichtigsten Herkunftsländer für ausländisches wissenschaftliches und künstlerisches Personal waren im Jahr 2019 Italien (3.582 bzw. 7,2\% der ausländischen Beschäftigten), China (3.089 bzw. 6,2\%) sowie Indien (2.932 bzw. 5,9\%). Zwischen 2015 und 2019 ist der Anteil Indiens deutlich und kontinuierlich von 4,7\% auf 5,9\% gestiegen. Leichte Anstiege sind auch für Iran (2015: 3,3\%, 2019: 3,7\%) sowie Italien (2015: 6,9\%, 2019: 7,2\%) zu beobachten. A Abb. 3.03

\section{Abbildung >> Anteil des wiss./künstl. Personals mit ausländischer Staatsbürgerschaft 3.01 am gesamten wiss./künstl. Personal nach Cluster, 2019}
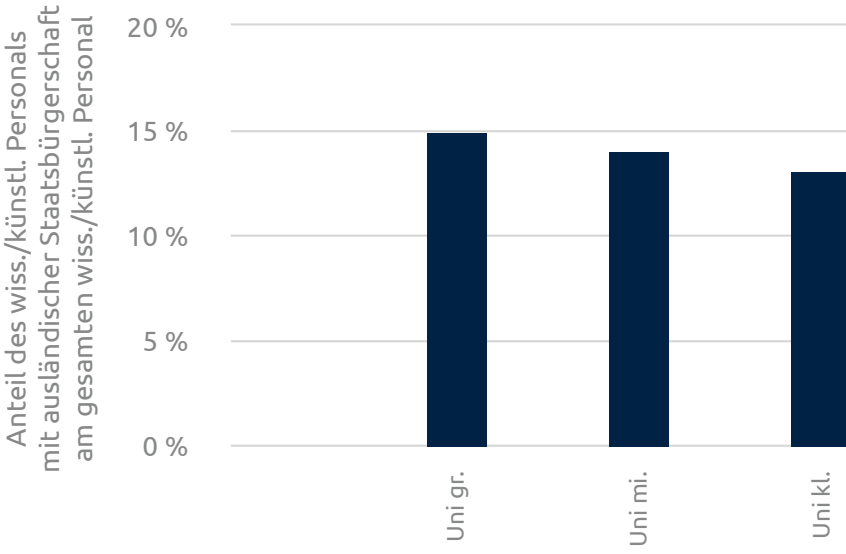

禀泀吉

2019

- Anteil des wiss./künstl. Personals mit ausländischer Staatsbürgerschaft am gesamten wiss./künstl. Personal 
Abbildung > Verteilung des wiss./künstl. Personals mit ausländischer Staatsbürgerschaft

3.02 nach Fächergruppe, 2016-2019

$25 \%$

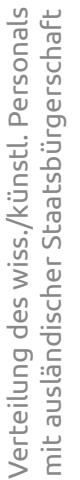

$20 \%$

$15 \%$

$10 \%$

$5 \%$

$0 \%$

Agrar-, Forst- und Ernährungs wissenschaften, Veterinärmedizin

- Geisteswissenschaften

- Ingenieurwissenschaften

Kunst, Kunstwissenschaft
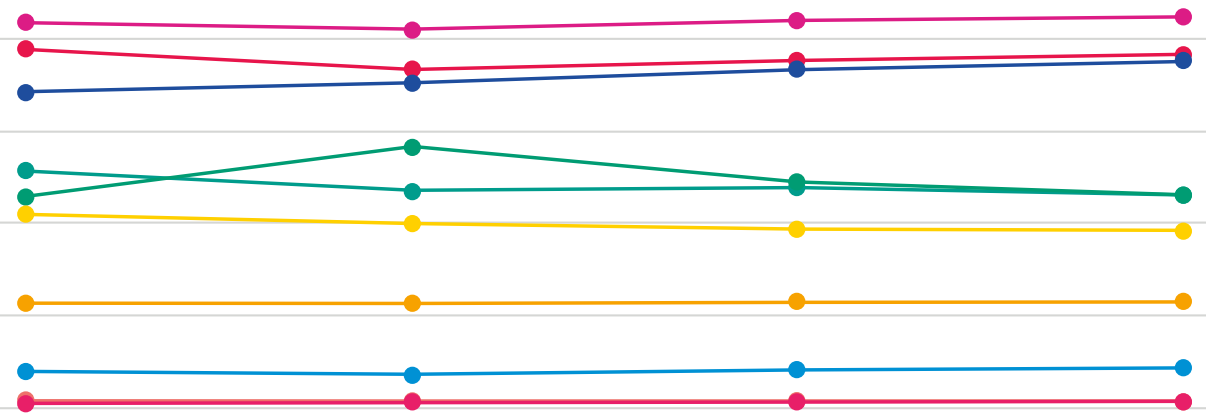

2017

2018

2019

Mathematik, Naturwissenschaften

Humanmedizin/Gesundheits-

wissenschaften

- Rechts-, Wirtschafts- und Sozial-

wissenschaften

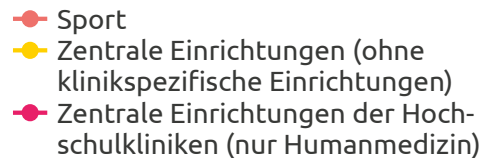

- Sport

schulkliniken (nur Humanmedizin)

Siehe auch: >> Gesamtzahl des wiss./künstl. Personals mit ausländischer Staatsbürgerschaft

nach Fächergruppe, 2016-2019

Abbildung >> Verteilung des wiss./künstl. Personals mit ausländischer Staatsbürgerschaft 3.03 nach Herkunftsland, 2015-2019

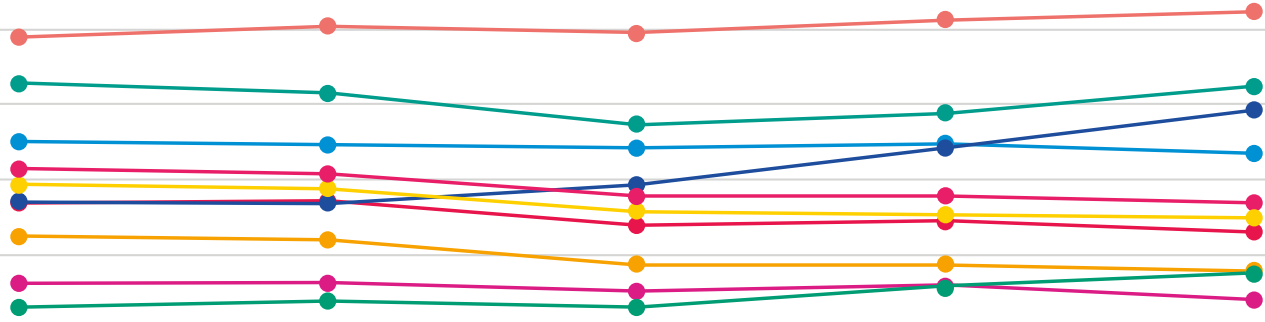

Österreich

- Vereinigtes Königreich

- Russische Föderation

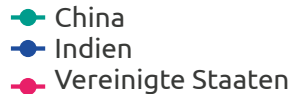

- Spanien

- Iran, Islamische

Republik
- Frankreich

- Italien

Siehe auch: >> Gesamtzahl des wiss./künstl. Personals mit ausländischer Staatsbürgerschaft nach Herkunftsland, 2015-2019 
Abbildung > Anteil der Professorinnen und Professoren mit ausländischer Staatsbürgerschaft an allen Professorinnen und Professoren nach Cluster, 2019

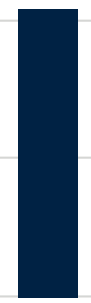

高

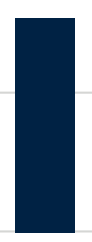

$\cdot \frac{\dot{\bar{E}}}{\grave{\supset}}$

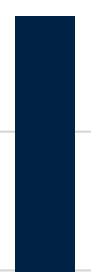

$\stackrel{\vec{v}}{\bar{c}}$

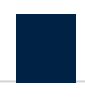

家

2019

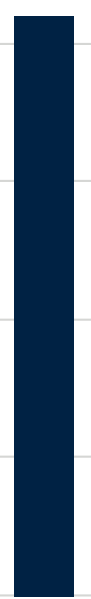

$\sum_{\underline{I}}^{I}$

- Anteil der Professor*innen mit ausländischer Staatsbürgerschaft an allen Professor*innen

Abbildung >> Verteilung der Professorinnen und Professoren mit ausländischer Staatsbürgerschaft 3.05 nach Herkunftsland, 2015-2019

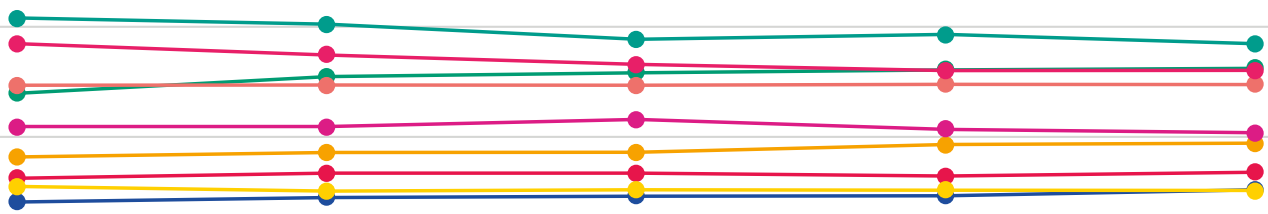




\subsection{Professorinnen und Professoren}

Im Jahr 2019 waren 3.416 Professorinnen und Professoren mit ausländischer Staatsbürgerschaft an den deutschen Hochschulen tätig, das bedeutete 5,2\% mehr als im Vorjahr.

\section{Im Detail:}

>> Professorinnen und Professoren mit ausländischer Staatsbürgerschaft im Zeitverlauf

Professorinnen und Professoren mit ausländischer Staatsbürgerschaft stellten im Jahr 2019 einen Anteil von insgesamt 7,1\% an allen Professorinnen und Professoren an deutschen Hochschulen. Bei der Betrachtung der einzelnen Hochschulcluster findet sich der höchste Anteil mit 21\% bei den Kunst- und Musikhochschulen. Universitäten wiesen im Durchschnitt einen Anteil von 9,6\% auf, Fachhochschulen einen vergleichsweisen niedrigen Anteil von 2,6\%. Abb. 3.04

Die meisten Professorinnen und Professoren mit ausländischer Staatsbürgerschaft waren 2019 in der Fächergruppe Mathematik/Naturwissenschaften tätig (757 bzw. 22,2\% aller ausländischen Professorinnen und Professoren), gefolgt von den Rechts-, Wirtschaftsund Sozialwissenschaften (619 bzw. 18,1\%) und Kunst/ Kunstwissenschaft (604 bzw. 17,7\%). Die Verteilung zwischen den Fächergruppen war in den letzten Jahren relativ konstant. ${ }^{4}$
Im Detail:

>> Gesamtzahl der Professorinnen und Professoren mit ausländischer Staatsbürgerschaft nach Fächergruppe, 2016-2019

> Verteilung der Professorinnen und Professoren mit ausländischer Staatsbürgerschaft nach Fächergruppe, 2016-2019

Bei den Herkunftsländern der Professorinnen und Professoren mit ausländischer Staatsbürgerschaft dominieren die europäischen Länder. Die meisten ausländischen Professorinnen und Professoren kamen 2019 aus Österreich (675 bzw. 19,8\%), der Schweiz (314 bzw. 9,2\%) und Italien (276 bzw. 8,1\%). Die USA lagen, als einziges (rein) außereuropäisches Land unter den zehn wichtigsten Herkunftsländern, mit 273 bzw. $8 \%$ der ausländischen Professorinnen und Professoren auf Platz vier. Russland belegte, als transkontinentales europäisch-asiatisches Land, Platz 10 (88 bzw. 2,6\% der ausländischen Professorinnen und Professoren). Die Anteile der zehn wichtigsten Herkunftsländer haben sich seit 2015 nur geringfügig geändert.

- Abb. 3.05 


\section{융 Erasmus-Mobilität}

- Die populärsten Zielländer für ausreisende Erasmus-Studierende waren im Jahr 2018 Spanien, Frankreich und Großbritannien; bei den ausreisenden Praktikantinnen und Praktikanten lag Großbritannien an erster Stelle vor Spanien und Frankreich.

- Die meisten einreisenden Erasmus-Studierenden kamen 2018 aus Italien, Frankreich und Spanien.

- Für ausreisendes Lehrpersonal waren im Jahr 2018 Italien, Spanien und Frankreich die populärsten Zielländer. Das einreisende Lehrpersonal kam vorrangig aus Spanien, gefolgt von den etwa gleich stark vertretenen Ländern Finnland, Italien, Frankreich und Großbritannien.

- Die Zahl besonders des ausreisenden nicht-wissenschaftlichen Personals steigt. 2018 verbrachten beinah doppelt so viele nichtwissenschaftliche Hochschulmitarbeiterinnen und -mitarbeiter (1.975) einen Aufenthalt im Ausland wie nicht-wissenschaftliche Mitarbeiterinnen und Mitarbeiter ausländischer Hochschulen nach Deutschland kamen (1.058). 


\section{Erasmus-Mobilität}

Als weltweit größtes Mobilitätsprogramm fördert Erasmus nicht nur die studienbezogene Mobilität von Studierenden, sondern auch Auslandspraktika sowie die Mobilität von Lehrenden und nicht-wissenschaftlichen Mitarbeiterinnen und Mitarbeitern. Im HSI-Monitor werden derzeit die Mobilitäten aus der Erasmus+ Key
Action 103 „Mobil in Europa” zusammengefasst, daher können Mobilitätsströme nur für den europäischen Raum ausgewertet werden.

\section{Lesen Sie mehr zum Thema unter: >> HSI-Monitor > Themen > Erasmus-Mobilität}

\subsection{Studierende}

Die Zahl der ausreisenden Studierenden im ErasmusProgramm hat im Erasmusjahr 2018 (entspricht dem Zeitraum 1. Juni 2017 - 31. Mai 2019) weiter zugenommen und liegt jetzt bei 33.104. Die Zahl der einreisenden Studierenden sinkt dagegen seit dem Erasmusjahr 2016 und ist von 23.372 auf 21.673 gefallen.

\section{Im Detail:}

>> Ausreisende und einreisende ErasmusStudierende, 2014-2018

Der höchste Anteil ausreisender Studierender an allen Studierenden findet sich bei den kleinen Universitäten (1,6\% der Studierenden), gefolgt von den Kunstund Musikhochschulen (1,5\%) und den mittelgroßen Universitäten (1,4\%). Der Anteil bei den Fachhochschulen ist mit durchschnittlich 0,8\% der Studierenden deutlich geringer. Abb. 4.01

Beim Anteil der einreisenden Studierenden liegen, gemessen an der Gesamtzahl der Studierenden, die Kunst- und Musikhochschulen mit 1,6\% weit vor den Universitäten (0,9\%) und den Fachhochschulen (0,5\%).

Abb. 4.02 , S. 36

Sowohl beim Anteil der ausreisenden wie auch der einreisenden Studierenden an allen Studierenden schneiden kleine Universitäten und Fachhochschulen generell besser ab als die mittelgroßen und großen Hochschulen gleichen Typs. Eine Ausnahme bilden die mittelgroßen Fachhochschulen, die beim Anteil der ausreisenden Studierenden mit 1,1\% deutlich vor den kleinen und den großen Fachhochschulen liegen (0,8\% bzw. 0,7\%).

\section{Abbildung >> Anteil der ausreisenden Studierenden an allen Studierenden nach Cluster, 2018 4.01}

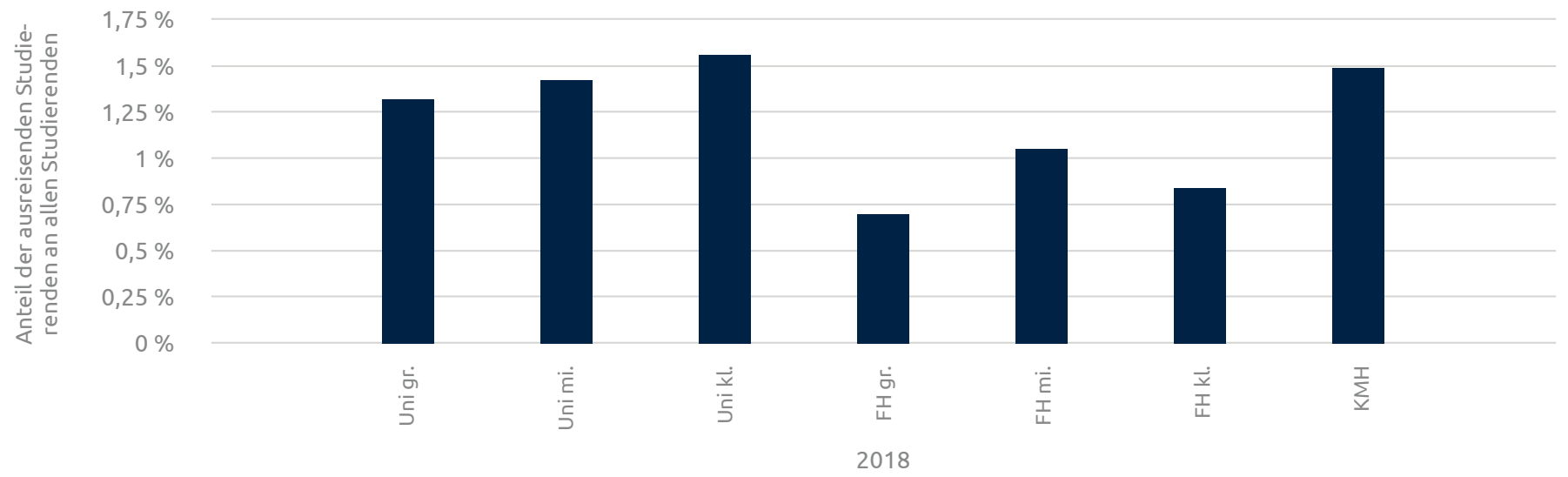

- Anteil der ausreisenden Studierenden an allen Studierenden 
Abbildung >> Anteil der einreisenden Studierenden an allen Studierenden nach Cluster, 2018 4.02

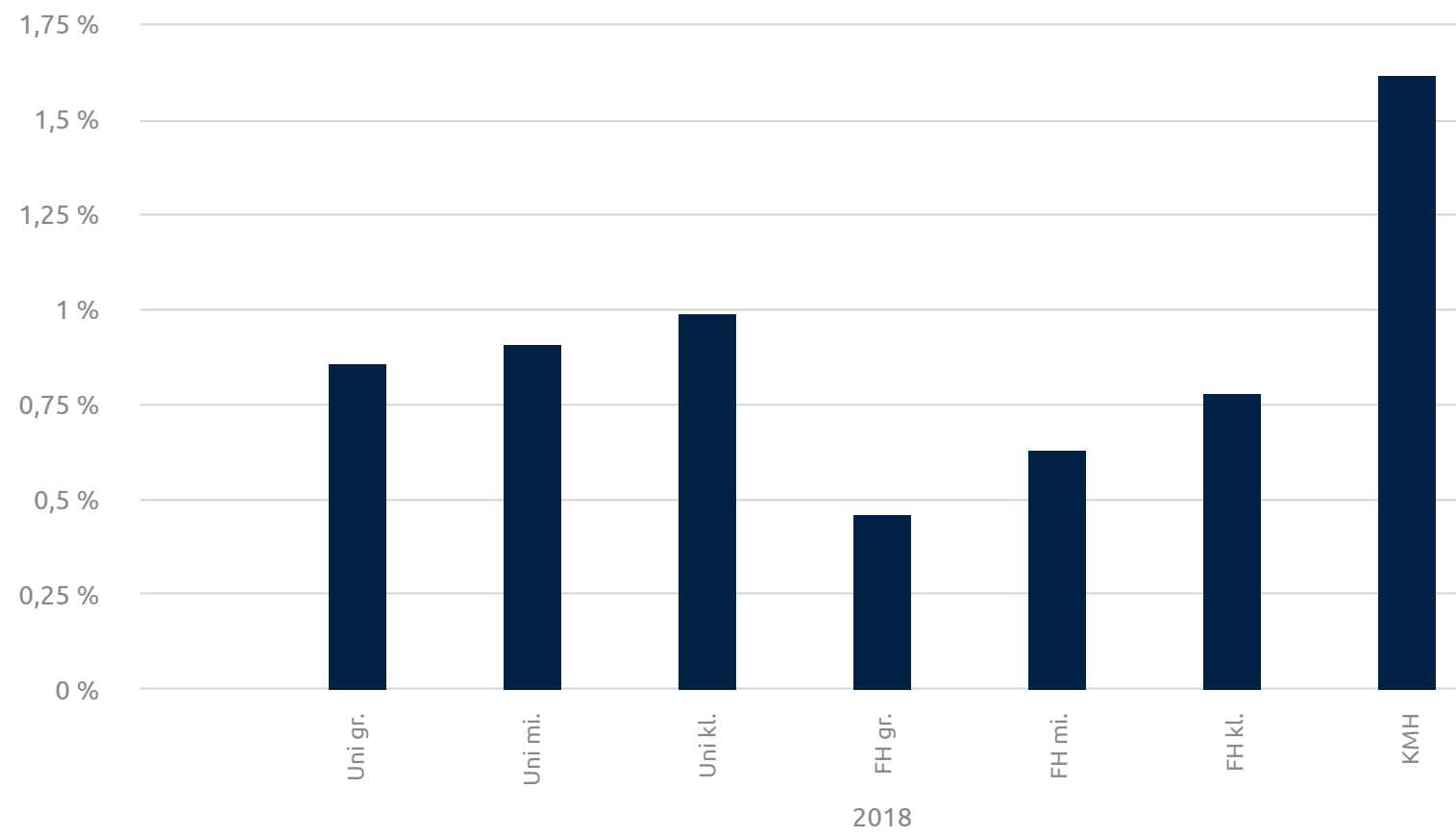

- Anteil der einreisenden Studierenden an allen Studierenden

Abbildung > Ausreisende Studierende nach Zielland, 2014-2018

4.03

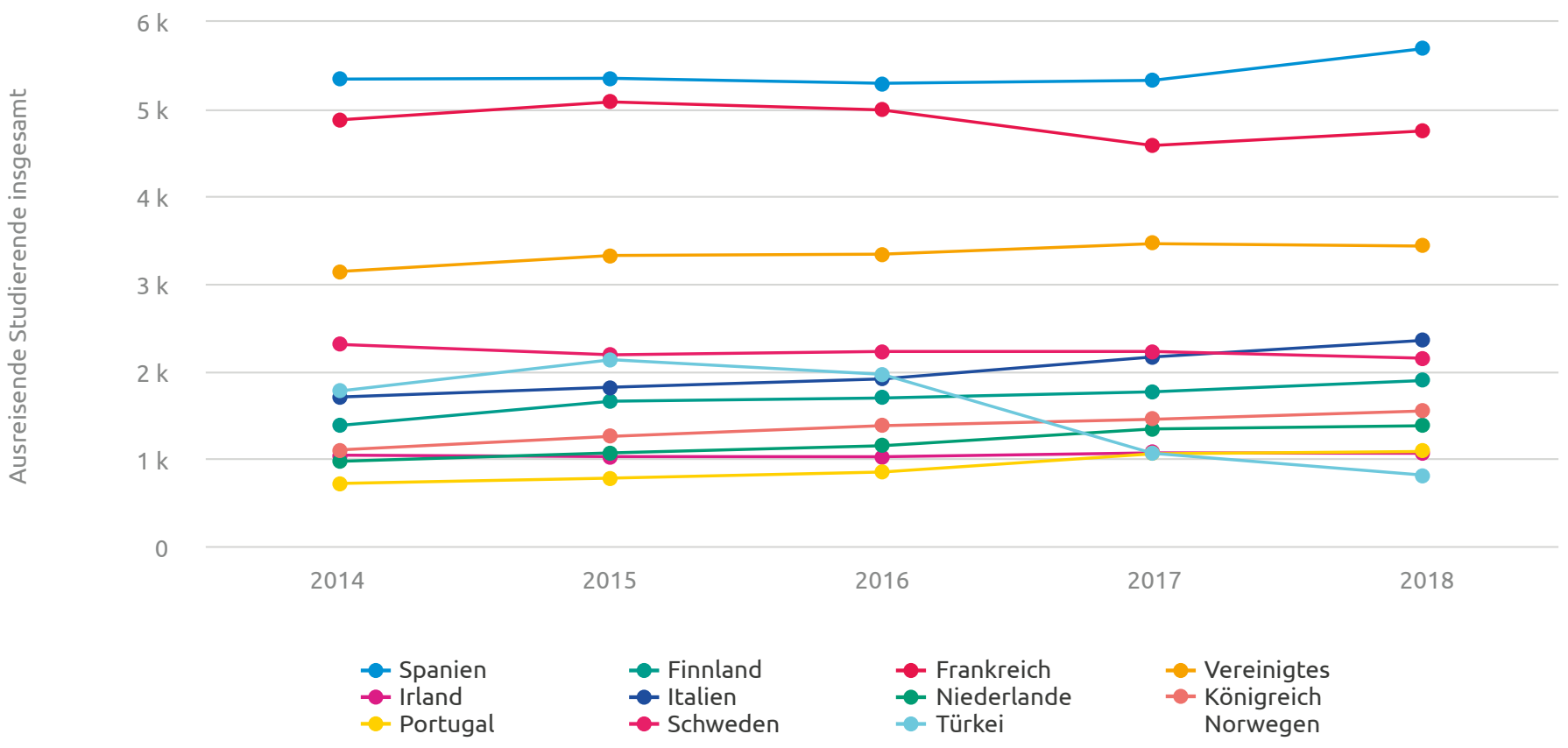

Siehe auch: >> Verteilung der ausreisenden Studierenden nach Zielland, 2014-2018 
Die meisten auslandsmobilen Studierenden im

Erasmus-Programm kamen 2018 aus der Fächergruppe der Rechts-, Wirtschafts- und Sozialwissenschaften (17.000 Studierende bzw. 51,4\% aller ausreisenden Studierenden). Daran schlossen sich die Geisteswissenschaften (5.696 bzw. 15,4\%) und die Ingenieurswissenschaften (4.048 bzw. 12,2\%) an.

\section{Im Detail:}

\section{> Ausreisende Studierende}

nach Fächergruppe, 2014-2018

\section{> Verteilung der ausreisenden Studierenden nach Fächergruppe, 2014-2018}

Die populärsten Zielländer für ausreisende Studierende aus Deutschland waren im Erasmusjahr 2018 Spanien (5.698 Studierende bzw. 17,2\% aller ausreisendenden Studierenden), gefolgt von Frankreich (4.748 bzw. 14,3\%) und Großbritannien (3.437 Studierende bzw. 10,4\%). Auffällig ist, dass die Zahl der Studierenden mit Ziel Türkei zwischen dem Erasmusjahr 2015 und 2018 von 2.139 auf 812 Studierende immens gefallen ist.

Abb. 4.03
Auch bei den einreisenden Studierenden sind die Rechts-, Wirtschafts- und Sozialwissenschaften mit 8.925 Studierenden (41,2\% aller einreisenden Studierenden) am stärksten vertreten, vor den Geisteswissenschaften (5.004 bzw. 23,1\%) und den Ingenieurswissenschaften (3.590 bzw. 16,6\%).

\section{Im Detail:}

>> Einreisende Studierende nach Fächergruppe, 2014-2018

\section{>> Verteilung der einreisenden Studierenden nach Fächergruppe, 2014-2018}

Die meisten Studierenden, die 2018 über das ErasmusProgramm nach Deutschland einreisten, kamen aus Italien (3.386 bzw. 15,6\% der einreisenden Studierenden), Frankreich (3.355 bzw. 15,5\%) und Spanien (2.874 bzw. 13,3\%). Zwischen 2014 und 2018 hat der Anteil der einreisenden Studierenden aus Italien am meisten zugenommen, von 11\% auf 15,6\%. Die Zahl der einreisenden Studierenden aus Spanien ist im gleichen Zeitraum um 2,1 Prozentpunkte gesunken. - Abb. 4.04

\section{Abbildung >> Einreisende Studierende nach Herkunftsland, 2014-2018

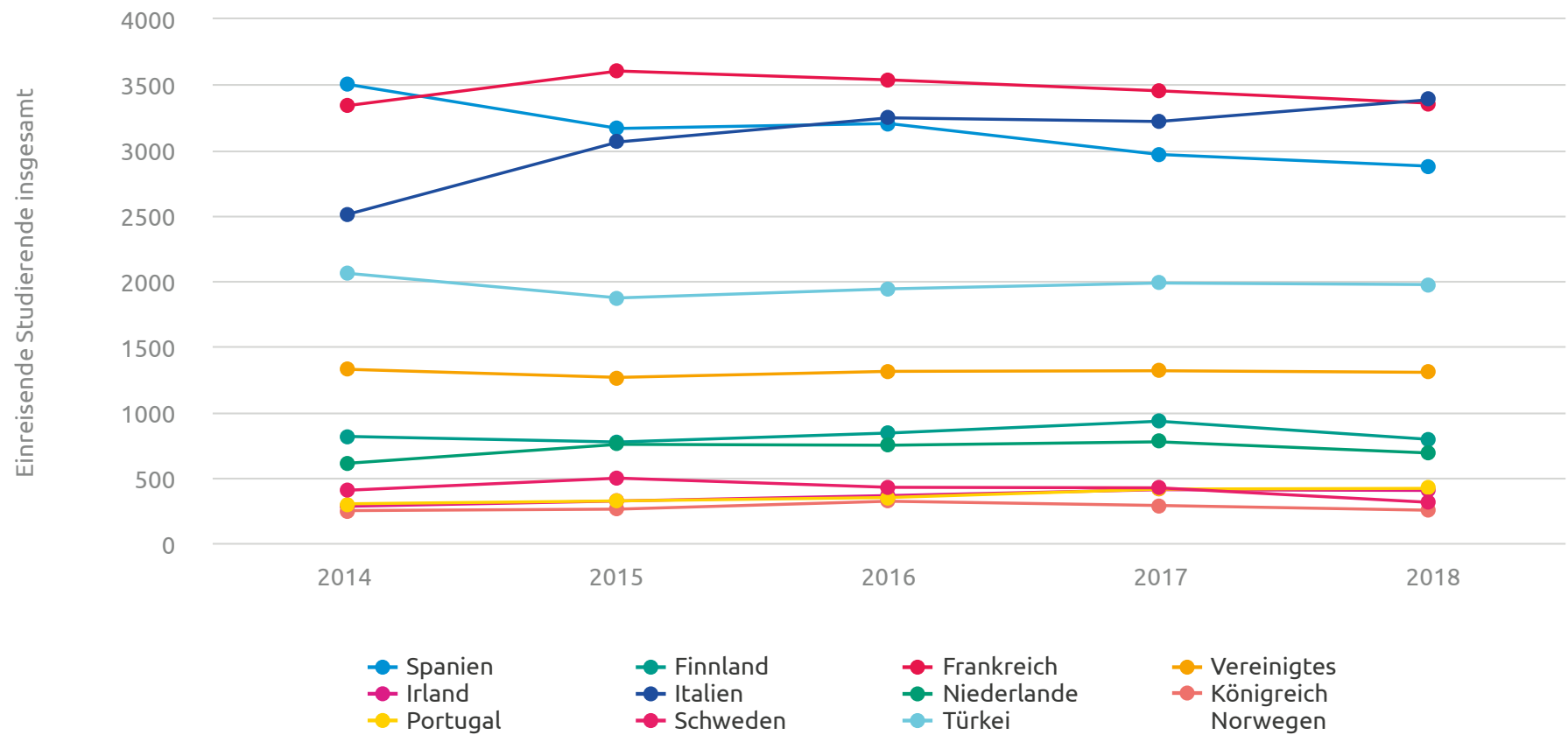




\subsection{Praktikantinnen und Praktikanten}

Die Zahl der ausreisenden Praktikantinnen und Praktikanten ist weiter gestiegen von $8.491 \mathrm{im}$ Erasmusjahr 2017 auf 8.867 im Erasmusjahr 2018.

\section{Im Detail:}

>> Ausreisende Praktikantinnen

und Praktikanten, 2014-2018

Der Anteil der ausreisenden Praktikantinnen und Praktikanten an allen Studierenden war 2018 beim Cluster der kleinen Fachhochschulen mit 0,7\% besonders hoch, es folgten die kleinen Universitäten (0,5\%) und die mittelgroßen Fachhochschulen (0,4\%). Nach Hochschultyp getrennt liegen Fachhochschulen und Kunst- und Musikhochschulen mit jeweils 0,4\% vor den Universitäten (0,3\%). A Abb. 4.05

\section{Beinah die Hälfte (47,1\%) der 2018 ausreisenden} Praktikantinnen und Praktikanten studierte ein Fach der Rechts-, Wirtschafts- und Sozialwissenschaften. Es schlossen sich die Mathematik/Naturwissenschaften $(14,5 \%)$, die Ingenieurswissenschaften $(12,5 \%)$ und die Humanmedizin/Gesundheitswissenschaften (10,0\%) an.
Im Detail:

>> Ausreisende Praktikantinnen und

Praktikanten nach Fächergruppe, 2014-2018

>> Verteilung der ausreisenden Praktikantinnen und Praktikanten nach Fächergruppe, 2014-2018

Populärstes Zielland für Praktikantinnen und Praktikanten war 2018 weiterhin Großbritannien (1.753 Praktikantinnen und Praktikanten bzw. 19,8\%), vor Spanien (1.321 bzw. 14,9\%) und Frankreich (870 bzw. 9,8\%). Der Anteil der Praktikantinnen und Praktikanten mit Ziel Niederlande ist zwischen 2014-2018 um 2,9 Prozentpunkte gewachsen; leicht zurückgegangen sind die Anteile der Praktikantinnen und Praktikanten, die in Großbritannien und der Türkei ein Praktikum absolvierten (Rückgang um 1,7 bzw. 1,6 Prozentpunkte).

Im Detail:

>> Ausreisende Praktikantinnen und Praktikanten nach Zielland, 2014-2018

>> Verteilung der ausreisenden Praktikantinnen und Praktikanten nach Zielland, 2014-2018

\section{Abbildung > Anteil der ausreisenden Praktikantinnen und Praktikanten 4.05 an allen Studierenden nach Cluster, 2018}

\section{$0,7 \%$}

$0,6 \%$

$0,5 \%$

$0,4 \%$

$0,3 \%$

$0,2 \%$

$0,1 \%$

$0 \%$

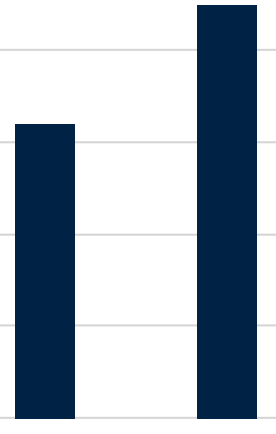

岕㝘 $\quad \dot{\vec{\varepsilon}}$
$\frac{\dot{\vec{v}}}{\dot{\vec{S}}}$

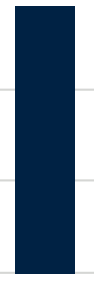

崩

2018

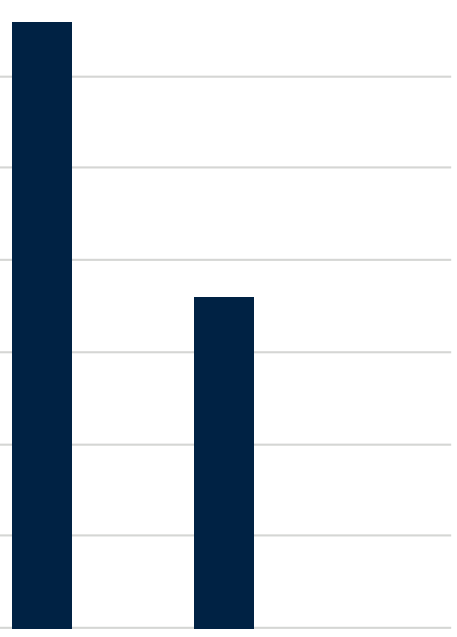

$\underset{⿱ 亠 䒑}{\dot{I}} \quad \frac{I}{\dot{I}}$ 


\subsection{Hochschulpersonal: Lehrkräfte}

Die Zahlen der ausreisenden und einreisenden

Lehrkräfte haben sich seit 2015 nur wenig verändert. Im Erasmusjahr 2018 reisten 3.097 Lehrkräfte aus Deutschland aus und 2.724 reisten nach Deutschland ein.

\section{Im Detail:}

>> Ausreisende und einreisende Lehrkräfte, 2015-2018

Ähnlich wie bei der Studierendenmobilität entfällt das meiste mobile Lehrpersonal, mit absteigender Tendenz, auf die Fächergruppen der Rechts-, Wirtschafts- und Sozialwissenschaften (im Erasmusjahr 2018 37,2\% des ausreisenden bzw. 36,2\% des einreisenden Lehrpersonals), dann der Geisteswissenschaften (ausreisend: 23,4\%, einreisend: 23,7\%) und der Ingenieurswissenschaften (ausreisend: 14,7\%, einreisend: 16,8\%).

Im Detail:

>> Ausreisende Lehrkräfte

nach Fächergruppe, 2014-2018

>> Verteilung der ausreisenden Lehrkräfte nach Fächergruppe, 2014-2018

>> Einreisende Lehrkräfte

nach Fächergruppe, 2014-2018

>> Verteilung der einreisenden Lehrkräfte nach Fächergruppe, 2014-2018
Die beliebtesten Zielländer für mobiles Lehrpersonal waren im Jahr 2018 Italien (367 Lehrkräfte bzw. 11,9\% aller ausreisenden Lehrkräfte) und Spanien (365 bzw. 11,8\%), Frankreich stand an dritter Stelle (281 bzw. 9,1\%). Die Popularität Italiens ist seit 2016 gestiegen, die Spaniens ist im Vergleich zu 2017 leicht gesunken. Die meisten Lehrkräfte, die 2018 nach Deutschland einreisten, stammten aus Spanien (231 bzw. 8,5\% des einreisenden Lehrpersonals); Finnland, Italien, Frankreich und Großbritannien lagen mit jeweils um die 180 Personen (6,7\%) etwa gleich auf.

\section{Im Detail:}

>> Ausreisende Lehrkräfte

nach Zielland, 2014-2018

>> Verteilung der ausreisenden

Lehrkräfte nach Zielland, 2014-2018

>> Einreisende Lehrkräfte

nach Herkunftsland, 2014-2018

>> Verteilung der einreisenden Lehrkräfte

nach Herkunftsland, 2014-2018

\subsection{Hochschulpersonal: Nicht-wissenschaftliches Personal}

Im HSI-Monitor wird erstmalig die Mobilität des nichtwissenschaftlichen Personals ausgewertet. Die Zahl des ausreisenden nicht-wissenschaftlichen Personals ist zwischen 2008 und 2018 kontinuierlich von 329 Personen auf 1.975 gestiegen. Auch die Zahl des einreisenden nicht-wissenschaftlichen Personals hat sich, mit leichten Schwankungen, zwischen 2009 und 2018 von 499 auf 1.058 Personen etwa verdoppelt. Aktuell verbringen annähernd doppelt so viele Hochschulmitarbeiterinnen und -mitarbeiter einen Aufenthalt im Ausland wie Mitarbeiterinnen und Mitarbeiter ausländischer Hochschulen nach Deutschland kommen. Abb. 4.06, S. 40
Kleinere Hochschulen weisen zwar niedrige Gesamtzahlen für ausreisendes und einreisendes nicht-wissenschaftliches Personal auf, gleichzeitig tendieren sie aber aufgrund der insgesamt geringeren personellen Ausstattung zu höheren Anteilen von mobilem nichtwissenschaftlichen Personal. Zwei Ausnahmen bilden, bezogen auf das ausreisende Personal, die mittelgroßen Fachhochschulen und die Kunst- und Musikhochschulen. Bei den mittelgroßen Fachhochschulen war im Jahr 2018 mit 326 ausreisenden nicht-wissenschaftlichen Beschäftigten die Zahl des mobilen Personals vergleichsweise hoch (an zweiter Stelle nach den großen Uni- 
versitäten mit 743 ausreisende Beschäftigten). Bei den Kunst- und Musikhochschulen hingegen war nicht nur die Gesamtzahl des 2018 ausgereisten Personals, sondern auch dessen Anteil an allen nicht-wissenschaftlichen Beschäftigen sehr niedrig. ${ }^{5}$

\section{Im Detail:}

>> Ausreisendes nicht-wissenschaftliches

Personal nach Cluster, 2018

>> Anteil des ausreisenden nicht-wissenschaftlichen Personals am gesamten nicht-wissenschaftlichen

Personal nach Cluster, 2018

\section{>> Einreisendes nicht-wissenschaftliches}

Personal nach Cluster, 2018

>> Anteil des einreisenden nicht-wissenschaftlichen Personals am gesamten nicht-wissenschaftlichen Personal nach Cluster, 2018
Großbritannien und Spanien erfreuen sich als Zielländer des ausreisenden nicht-wissenschaftlichen Personals zunehmender Beliebtheit. Die Zahl der Hochschulmitarbeiterinnen und -mitarbeiter, die einen Aufenthalt in Großbritannien absolvierten, hat sich zwischen 2014 und 2018 von 135 auf 355 mehr als verdoppelt, während sich die Zahl der Mitarbeiterinnen und Mitarbeiter mit Aufenthalt in Spanien zwischen 2014 und 2017 bei einer Zunahme von 97 auf 316 mehr als verdreifacht hat. Von 2017 auf 2018 ist die Zahl des nach Spanien ausgereisten nicht-wissenschaften Personals allerdings auf 288 gefallen. Die nächstpopulären Zielländer waren im Erasmusjahr 2018 Finnland, Schweden und Italien.

- Abb. 4.07

Zwischen 2017 und 2018 ist die Zahl des aus Frankreich einreisenden nicht-wissenschaftlichen Personals von 62 auf 96 stark gestiegen und lag damit an erster Stelle, vor Spanien (90), Großbritannien (78), der Türkei (76) und Finnland (57). Abb. 4.08

\section{Abbildung > Ausreisendes und einreisendes nicht-wissenschaftliches Personal im Zeitverlauf} 4.06

$$
\begin{aligned}
& 2400 \\
& 2000 \\
& 1600 \\
& 1200 \\
& 800 \\
& 400
\end{aligned}
$$$$
1600
$$$$
800
$$
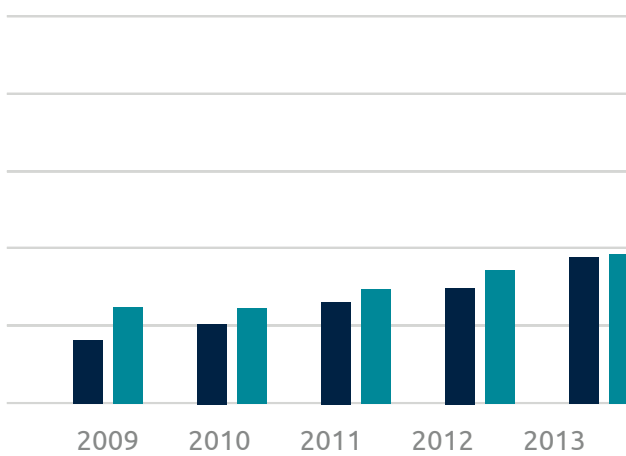

- (A) Ausreisendes nichtwissenschaftliches Personal insgesamt

- (B) Einreisendes nichtwissenschaftliches Personal insgesamt 
Abbildung > > Ausreisendes nicht-wissenschaftliches Personal nach Zielland, 2014-2018 4.07
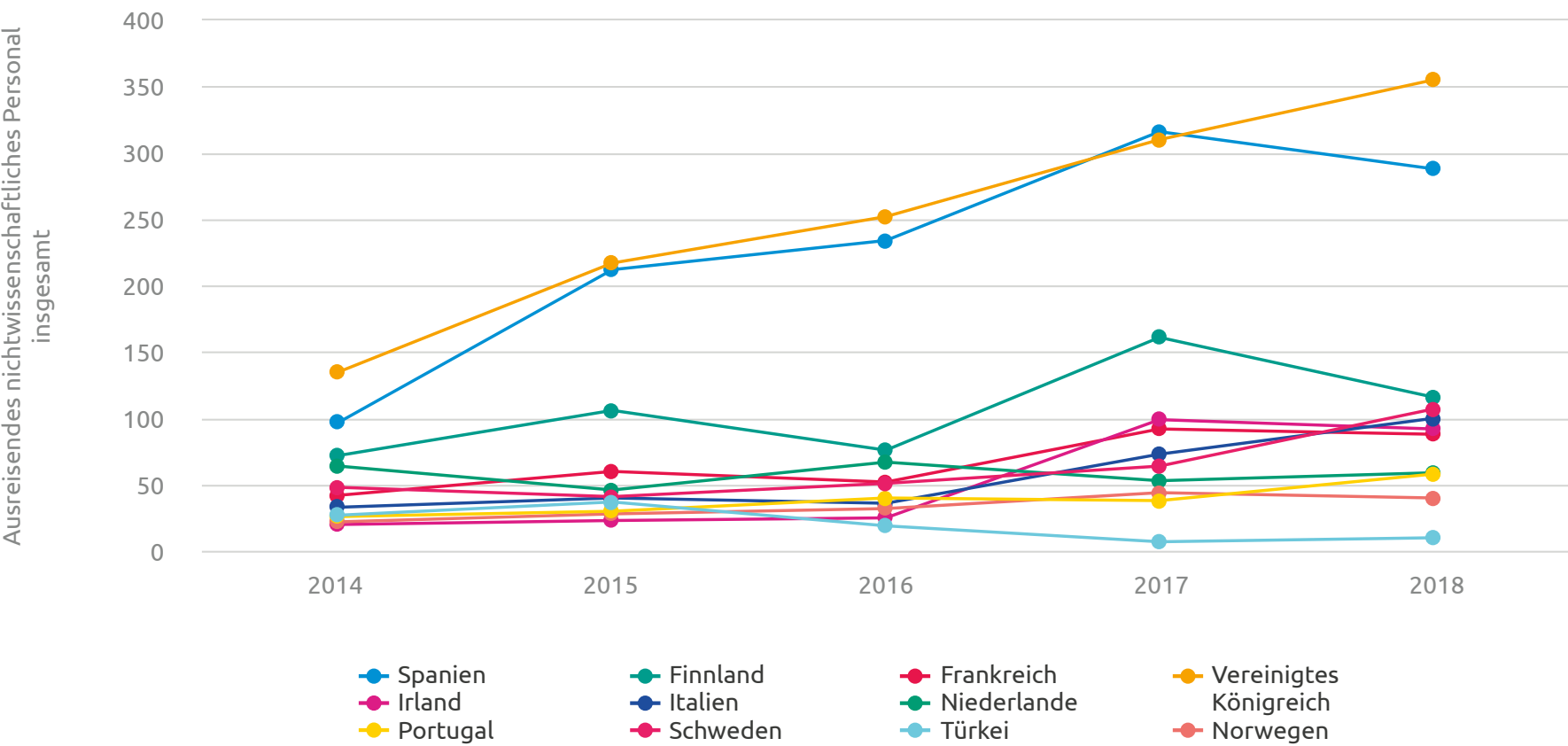

- Vereinigtes
Königreich
- Norwegen

Siehe auch: >> Verteilung des ausreisenden nicht-wissenschaftlichen Personals nach Zielland, 2014-2018

Abbildung >> Einreisendes nicht-wissenschaftliches Personal nach Herkunftsland, 2014-2018 4.08
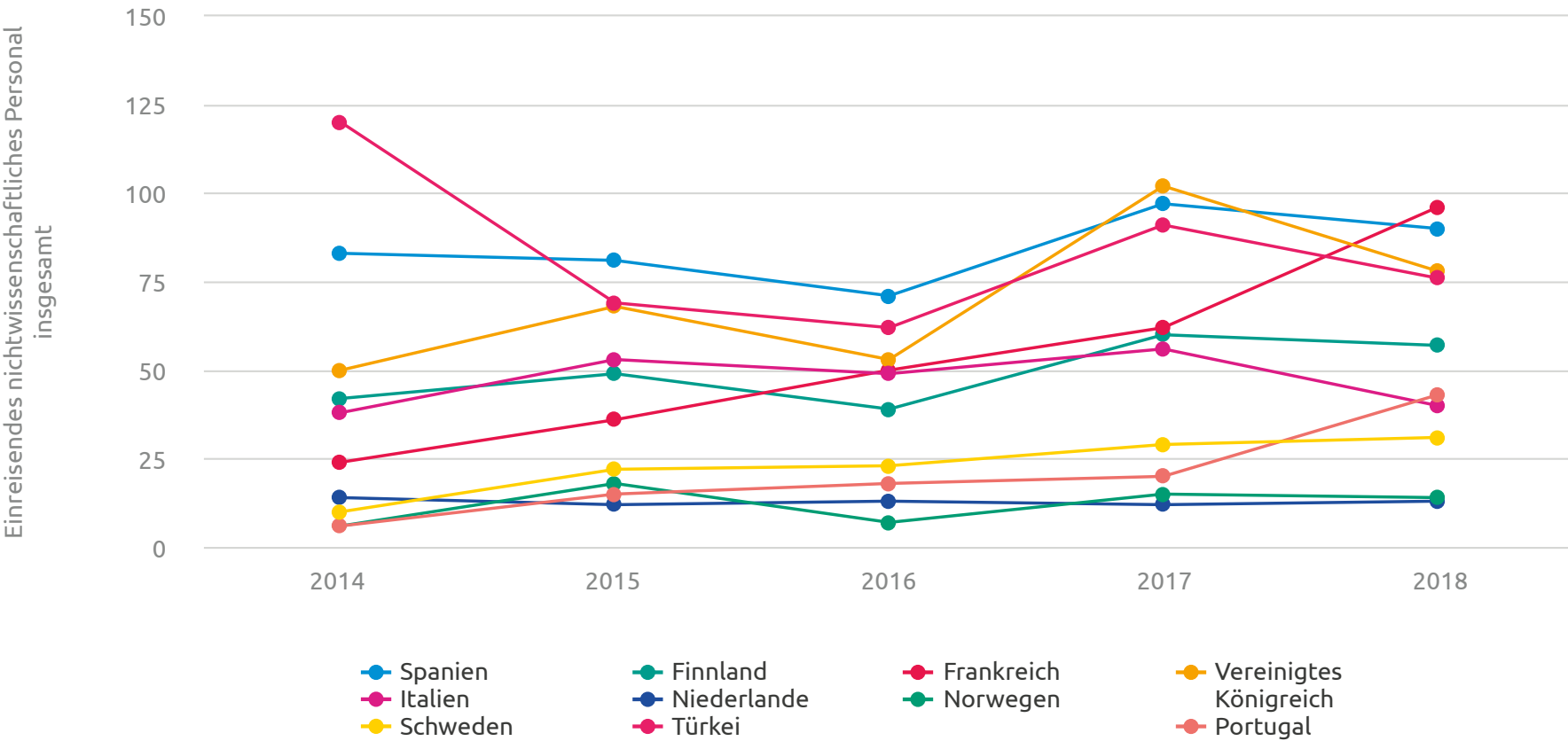
- Vereinigtes
Königreich
- Portugal

Siehe auch: >> Verteilung des einreisenden nicht-wissenschaftlichen Personals nach Herkunftsland, 2014-2018 


\section{- International ausgerichtete Studiengänge}

- Die Gesamtzahl der international ausgerichteten Studiengänge (2019: 2.370) und der englischsprachigen Studiengänge (2019: 1.420) an deutschen Hochschulen ist zwischen 2008 und 2019 beständig gestiegen.

- Der Anteil der international ausgerichteten Bachelor-Studiengänge ist bei etwa 6\% konstant geblieben; der Anteil der international ausgerichteten Master-Studiengänge hat deutlich zugenommen und lag 2019 bei rund $18 \%$.

- Sowohl auf dem Bachelor- als auch auf dem Master-Level hat der Anteil an englischsprachigen Studiengängen zugenommen, der Anteil auf dem Master-Level war 2019 dabei mehr als sechsmal so hoch wie auf dem Bachelor-Level (12,6\% im Vergleich zu 2,0\%).

- Die Anzahl der Doppelabschluss-Studiengänge hat sich seit 2009 knapp vervierfacht und lag 2019 bei 753 Studiengängen. Die beliebtesten Partnerländer sind Frankreich (438 Studiengänge), Großbritannien (131) und die USA (123). 


\section{International ausgerichtete Studiengänge}

Studiengänge mit internationaler Ausrichtung sind für Hochschulen ein wichtiges Instrument, um die Attraktivität für ausländische Studierende zu steigern und die Ausbildung der eigenen Studierenden zu internationalisieren. Als Informationsquelle zur Berechnung von Kennzahlen zu internationalen bzw. international ausgerichteten Studiengängen dient der Hochschulkompass der Hochschulrektorenkonferenz (HRK), in dem nicht nur sämtliche Studiengänge der deutschen Hochschulen verzeichnet sind, sondern auch internationale Studiengänge als solche gekennzeichnet werden können. Im HSI-Monitor werden zu- sätzlich zu den international ausgerichteten Studiengängen auch englischsprachige Studiengänge sowie gemeinsame Studiengänge von Hochschulen aus unterschiedlichen Ländern und mit Möglichkeit zum doppelten Abschluss ausgewertet. Beide Indikatoren stellen Untergruppen des Indikators ,International ausgerichtete Studiengänge" dar.

\section{Lesen Sie mehr zum Thema unter:}

>> HSI-Monitor > Themen > International ausgerichtete Studiengänge

\subsection{Internationale und englischsprachige Studiengänge insgesamt}

Die Gesamtzahl der internationalen und der englischsprachigen Studiengänge an deutschen Hochschulen ist zwischen 2008 und 2019 beständig gestiegen. Im Beobachtungszeitraum hat sich die Zahl der internationalen Studiengänge von 771 auf 2.370 verdreifacht, die Zahl der englischsprachigen Studiengänge hat sich von 258 auf 1.420 sogar mehr als verfünffacht.
Der höchste Anteil von internationalen Studiengängen an allen Studiengängen fand sich 2019 bei den kleinen Fachhochschulen (18,4\%), gefolgt von den mittelgroßen Fachhochschulen (13,5\%) und den mittelgroßen Universitäten (13,3\%). Abb. $\mathbf{5 . 0 1}$

Auch beim Anteil der englischsprachigen Studiengänge an allen Studiengängen liegen die kleinen Fachhochschulen mit 14,8\% deutlich vorn. Es folgen die kleinen Universitäten (8,3\%) und die großen Universitäten (8,2\%).

$\rightarrow$ Abb. 5.02, S. 44

Abbildung > Anteil der internationalen Studiengänge an allen Studiengängen nach Cluster, 2019 5.01

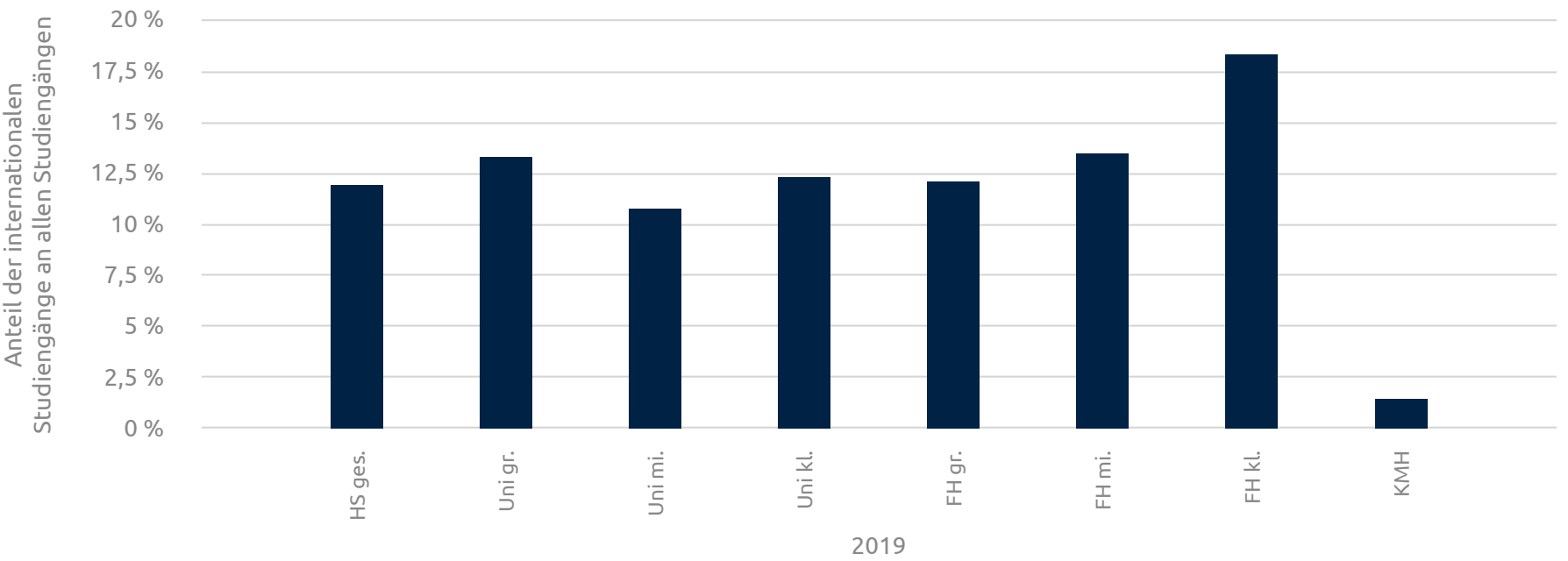

- Anteil der internationalen Studiengänge an allen Studiengängen 
Abbildung > Anteil der englischsprachigen Studiengänge

5.02 an allen Studiengängen nach Cluster, 2019
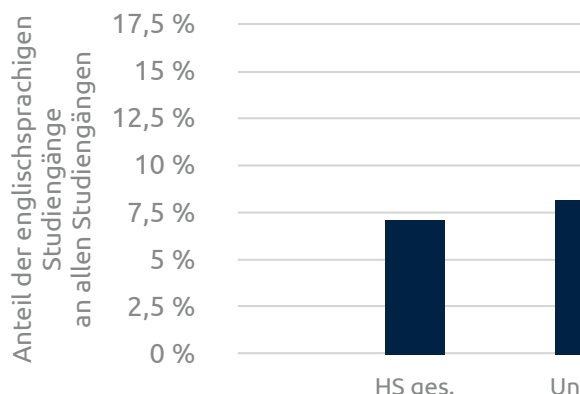

HS ges.
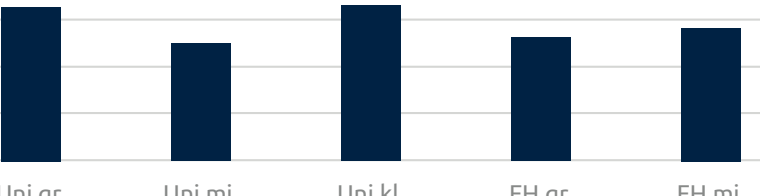

2019

- Anteil der englischsprachigen Studiengänge an allen Studiengängen

Abbildung

>> Anteil der internationalen Bachelor-Studiengänge

5.03 an allen Bachelor-Studiengängen nach Cluster, 2019
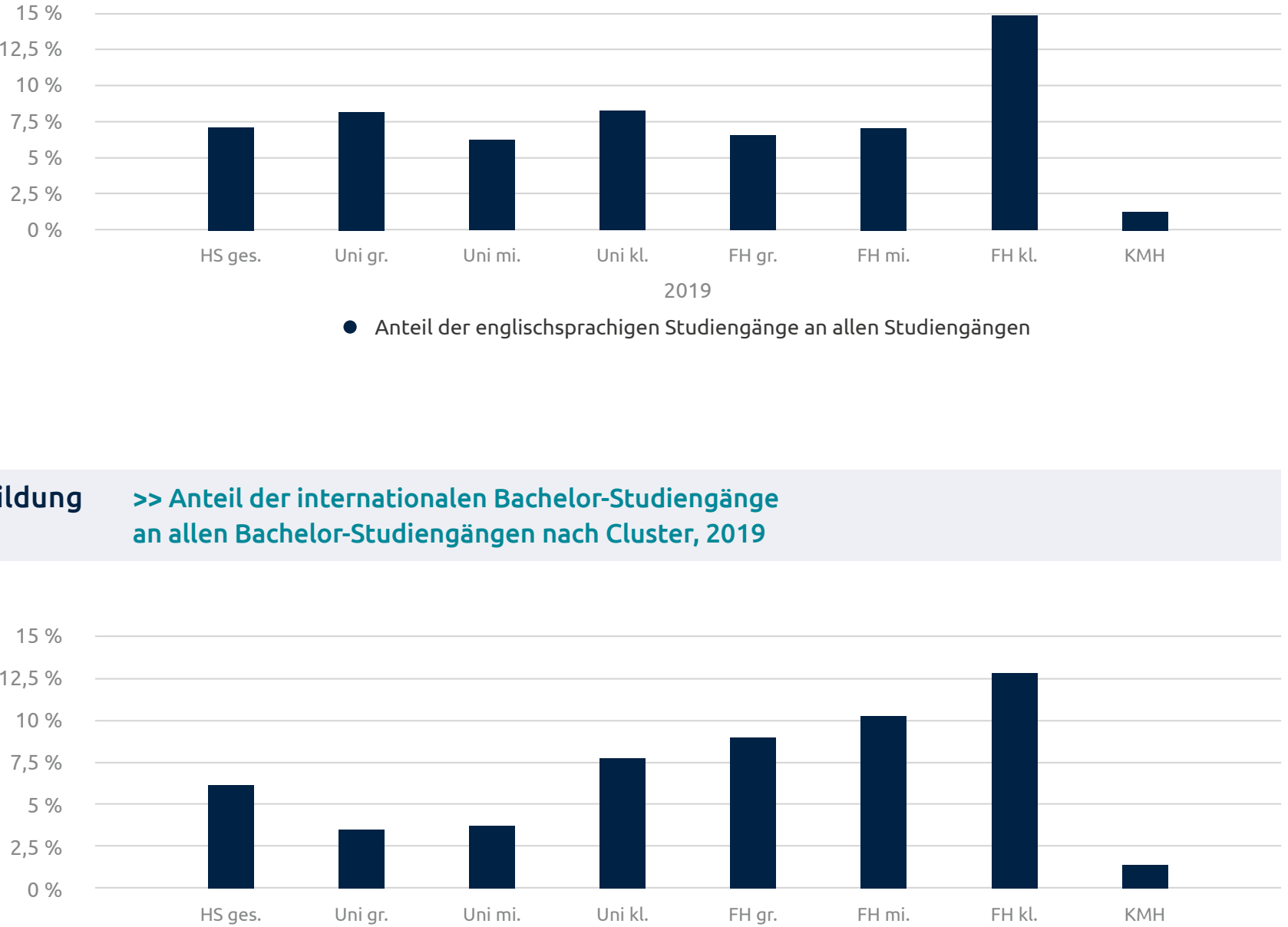

$\mathrm{KMH}$
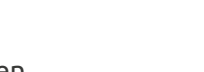


\subsection{Internationale und englischsprachige Studiengänge nach Abschluss}

Der Anteil der internationalen Studiengänge auf dem Bachelor-Level ist zwischen 2009 und 2019 bei etwa 6\% konstant geblieben. Der Anteil der internationalen Studiengänge auf Master-Niveau hat im Beobachtungszeitraum jedoch deutlich zugenommen; nach einem leichten Rückgang bis 2012 ist er zwischen 2009 und 2019 von 14,0\% auf 18,1\% gestiegen. Auf dem BachelorLevel ist der Anteil der internationalen Studiengänge am höchsten bei den kleinen, mittelgroßen und großen Fachhochschulen (12,8\%, 10,3\% bzw. 9,0\%). Auch die kleinen Universitäten liegen mit 7,8\% über dem Durchschnitt aller Hochschulen (6,2\%). A Abb. $\mathbf{5 . 0 3}$

Sowohl auf dem Bachelor-als auch auf dem Master-Level hat der Anteil der englischsprachigen Studiengänge zugenommen, auf dem Master-Level war dieser 2019 mehr als sechsmal so hoch wie auf dem Bachelor-Level (12,6\% im Vergleich zu 2,0\%).
Auch auf dem Master-Level liegen die kleinen Fachhochschulen mit einem Anteil von 28,8\% vorn. Es folgen die großen Universitäten mit 22,2\%. Abb. 5.04

Mit 9\% aller Bachelor-Studiengänge weisen die kleinen Fachhochschulen einen sehr viel höheren Anteil an englischsprachigen Studiengängen auf als die anderen Hochschulcluster. Es folgen die kleinen Universitäten mit 4,5\% und die mittelgroßen Fachhochschulen mit 3,1\%.

Abb. 5.05

\section{Abbildung > Anteil der englischsprachigen Bachelor-Studiengänge}

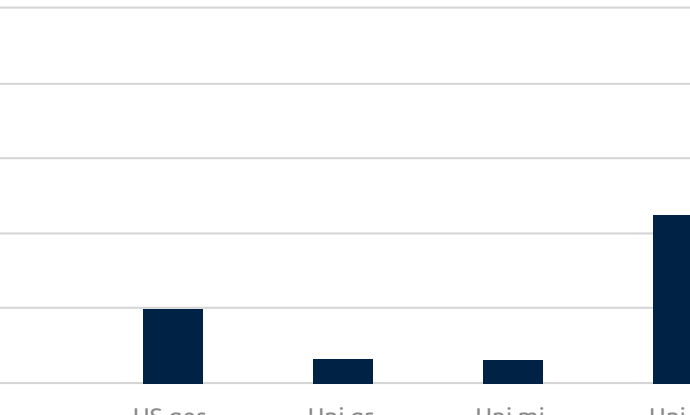

HS ges.

Uni gr.

Uni mi.

Uni kl.
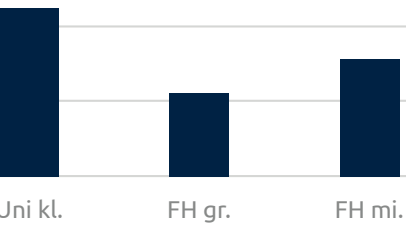

FH mi.

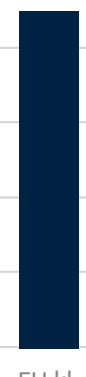

$\mathrm{FH} \mathrm{kl}$.

$\mathrm{KMH}$

- Anteil der englischsprachigen Bachelor-Studiengänge an allen Bachelor-Studiengängen 
Abbildung >> Anteil der englischsprachigen Master-Studiengänge

5.06 an allen Master-Studiengängen nach Cluster, 2019

$30 \%$

$25 \%$

$20 \%$

$15 \%$

$10 \%$

$5 \%$

$0 \%$

Abbildung

5.07

\begin{tabular}{l}
$80 \%$ \\
$70 \%$ \\
$60 \%$ \\
$50 \%$ \\
\hline $30 \%$ \\
\hline $20 \%$ \\
\hline $10 \%$ \\
\hline
\end{tabular}

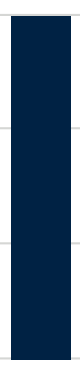

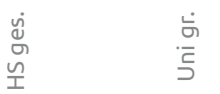

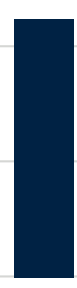

$\cdot \frac{\dot{E}}{\bar{D}}$

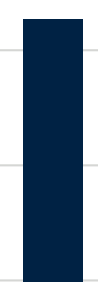

$\frac{\dot{x}}{\dot{\Xi}}$

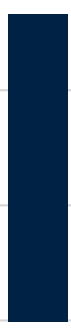

它

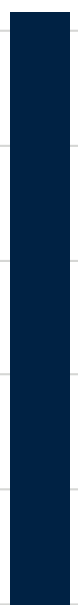

$\underset{\dot{⿱ 亠 䒑}}{\stackrel{\dot{I}}{\dot{I}}}$

2019

- Anteil der englischsprachigen Master-Studiengänge an allen Master-Studiengängen

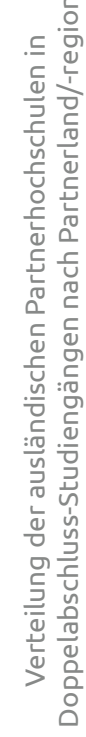

>> Verteilung der ausländischen Partnerhochschulen

in Doppelabschlussstudiengängen nach Partnerregion, 2015-2019
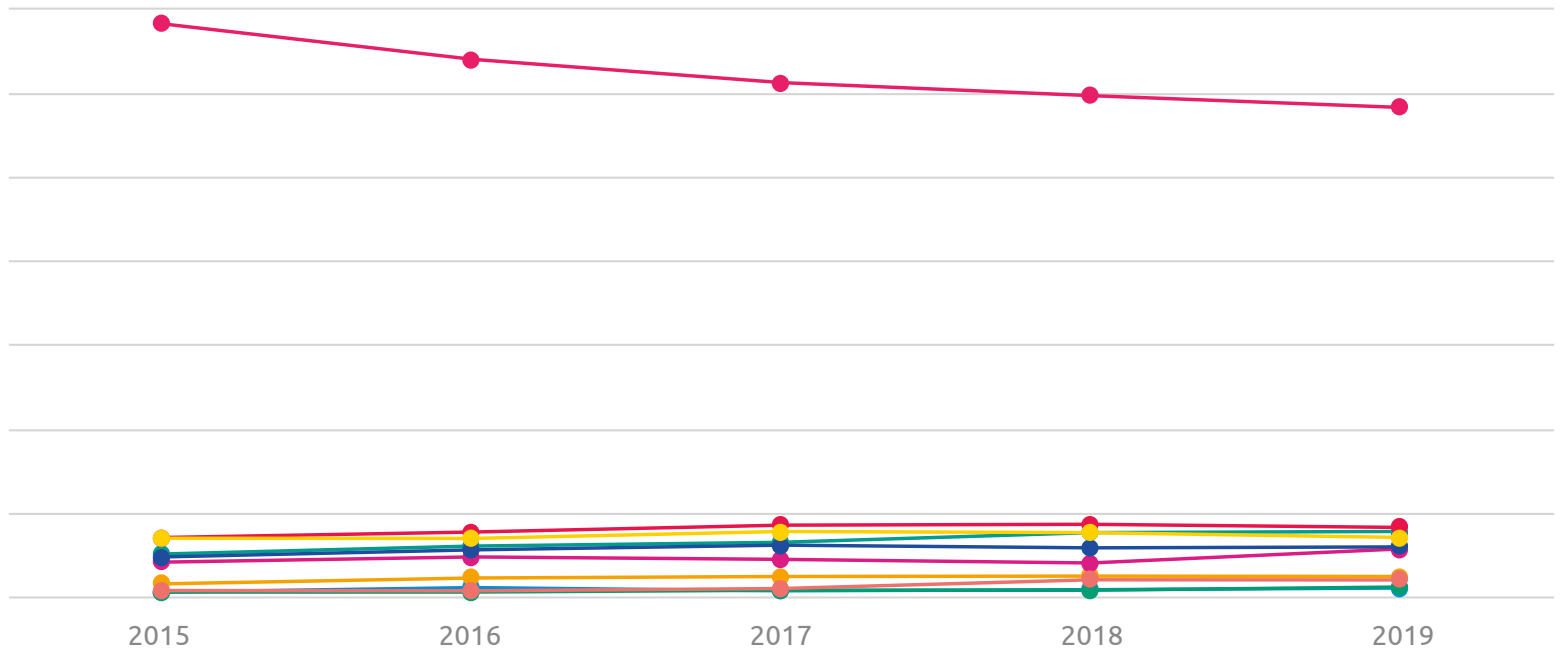

Subsahara-Afrika

Australien und Ozeanien

- Südasien

- Westeuropa
Nordamerika

Süd- und Mittelamerika

- Mittel- und Südosteuropa 


\subsection{Doppelabschlussstudiengänge}

Auch auf dem Master-Level liegen die kleinen Fachhochschulen mit einem Anteil von 25,6\% deutlich über den Anteilen der anderen Hochschulen. Die großen Universitäten und die großen Fachhochschulen folgen mit 15,0\% bzw. 13,4\%. Abb. 5.06
Die Anzahl der Studiengänge mit Doppelabschluss hat sich zwischen 2009 und 2019 von 191 auf 753 knapp vervierfacht. Bei der Verteilung der ausländischen Partnerhochschulen in Doppelabschlussstudiengängen nach Region liegt Westeuropa mit 58,2\% in 2019 weit vorn. Es folgen Nordamerika (8,2\%) und Ostasien (7,7\%). Die Region Westeuropa hat gleichwohl zwischen 2015 und 2019 an Bedeutung verloren. 2015 waren noch $68,1 \%$ aller ausländischen Partnerhochschulen in Westeuropa, wohingegen der Anteil 2019 nur noch 58,2\% betrug. Am stärksten gestiegen sind die Anteile der Regionen Ostasien (von 5,1\% auf 7,7\%), Osteuropa und Zentralasien (von 4,1\% auf 5,7\%) sowie Südostasien (von $0,7 \%$ auf $2,0 \%$ ). Abb. 5.07

Frankreich ist mit Abstand der beliebteste Kooperationspartner für Doppelabschlussstudiengänge, 2019 bestanden 438 davon mit französischen Hochschulen. Es folgten Großbritannien und die USA mit 131 bzw. 123 Doppelabschlussstudiengängen. Den größten Zuwachs gab es bei Kooperationen mit der Russischen Föderation, diese nahmen zwischen 2018 und 2019 um 40\% (von 50 auf 70 Studiengänge) zu. A Abb. $\mathbf{5 . 0 8}$

$\begin{array}{ll}\text { Abbildung } & \text { > Gesamtzahl der ausländischen Partnerhochschulen } \\ 5.08 & \text { in Doppelabschlussstudiengängen nach Land, 2015-2019 }\end{array}$
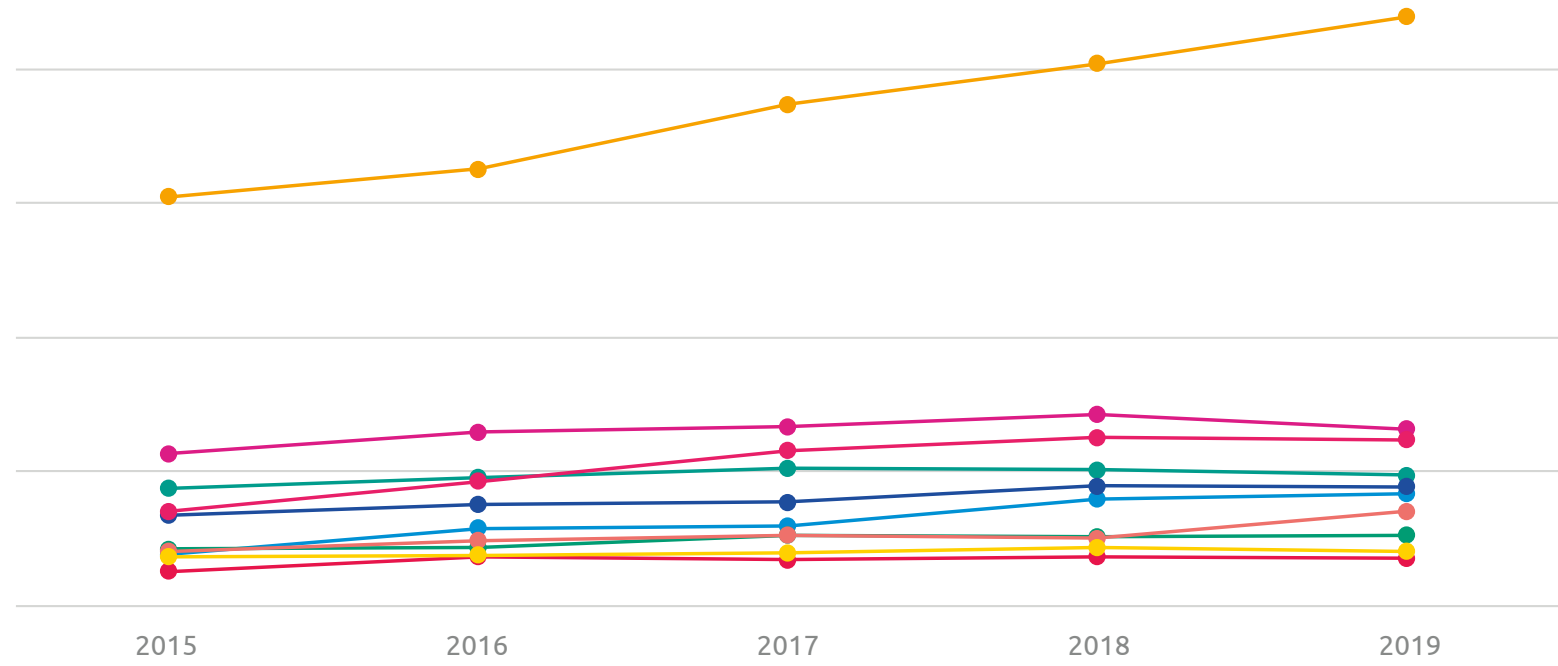


\section{(6) Internationale Hochschulkooperationen}

- Die Anzahl der internationalen Hochschulkooperationen (ohne Kooperationen im Erasmus-Programm) ist weiter gestiegen auf 17.033 im Jahr 2019.

- Die größte Anzahl der Kooperationen findet sich bei den großen Universitäten (5.486), gefolgt von den mittelgroßen Universitäten (3.945) und den mittelgroßen Fachhochschulen (3.005).

- Kooperationen innerhalb Europas (außerhalb des ErasmusProgramms) sind zurückgegangen zugunsten von Kooperationen mit Ostasien sowie Süd- und Mittelamerika. 


\section{Internationale Hochschulkooperationen}

Eine partnerschaftliche Zusammenarbeit mit Hochschulen und Forschungseinrichtungen im Ausland ist von großer Bedeutung für eine erfolgreiche, an internationalen Leistungsstandards orientierte Entwicklung von Forschung und Lehre. Die meisten deutschen Hochschulen unterhalten ein dichtes Netz an Kooperationen mit internationalen Partnereinrichtungen. Die internationalen Kooperations- abkommen werden im Informationsportal "Internationale Hochschulkooperationen" der Hochschulrektorenkonferenz (HRK) systematisch erfasst.

Lesen Sie mehr zum Thema unter: >> Informationsportal „Internationale Hochschulkooperationen" 
Abbildung >> Internationale Kooperationen (ohne Erasmus) nach Cluster 6.01 im Zeitverlauf: Große und mittelgroße Unis und FHs

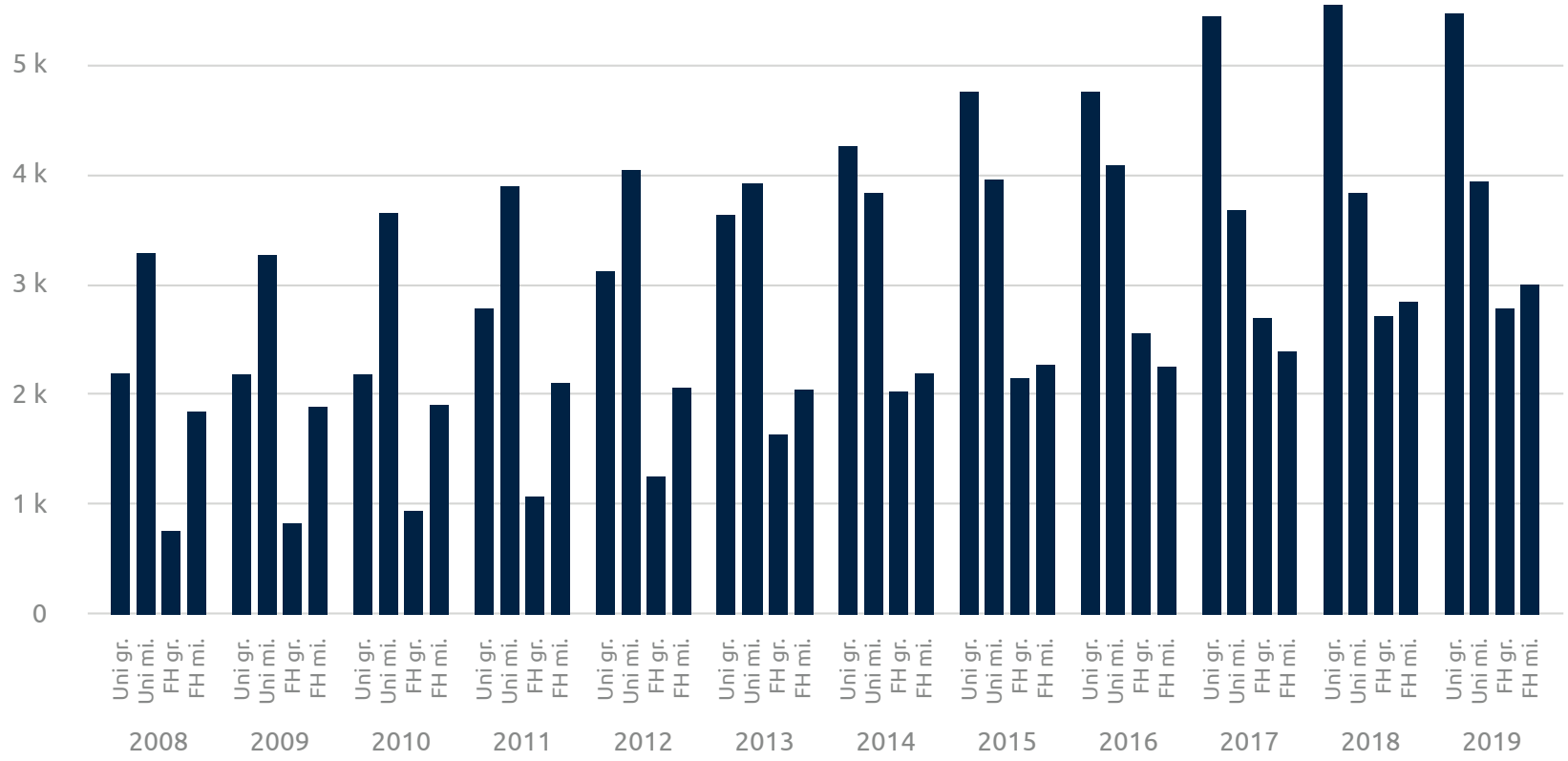

- Internationale Kooperationen (ohne Erasmus) insgesamt

Abbildung 6.02
>> Internationale Kooperationen (ohne Erasmus) nach Cluster im Zeitverlauf: Kleine Unis und FHs sowie KMHs

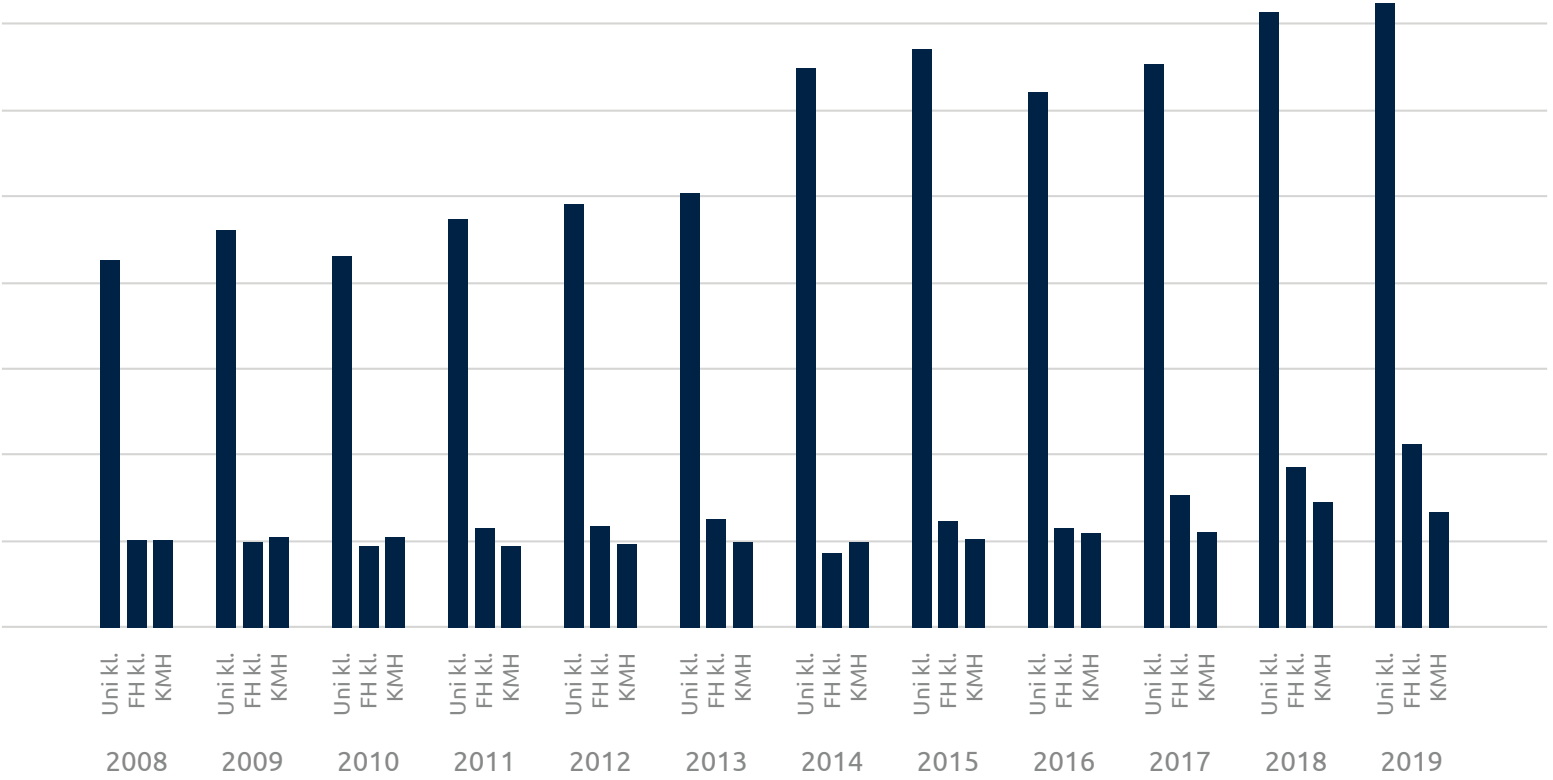




\title{
6.1 Grunddaten
}

Seit dem Beginn des Beobachtungszeitraums hat die Zahl der internationalen Hochschulkooperationen beständig zugenommen. Zwischen 2008 und 2019 ist die Zahl der Kooperationen außerhalb des Erasmus-

Programms von 9.726 auf 17.933 gestiegen, was einer Zunahme um 84,4\% entspricht. 6

\section{Im Detail:}

\section{>> Internationale Kooperationen} (ohne Erasmus) im Zeitverlauf

Die Analyse nach Hochschulclustern zeigt, dass der Zuwachs bei den großen Universitäten und den großen Fachhochschulen besonders stark war. Bei den großen Universitäten stieg die Zahl der internationalen Kooperationen zwischen 2008 und 2019 von 2.196 auf 5.486, was einer Zunahme um knapp 150\% entspricht. Noch stärker ist dies bei den großen Fachhochschulen zu beobachten (von 765 auf 2.788 Hochschulkooperationen, eine Zunahme um 264\%). Bei den mittelgroßen Universitäten nahm die Zahl der internationalen Partnerschaften von 3.300 auf 3.945 (Zunahme um knapp 20\%), bei den mittelgroßen Fachhochschulen von 1.849 auf 3.005 (Zunahme um 63\%) zu. A Abb. 6.01
Auch bei den kleinen Fachhochschulen hat sich die Anzahl der internationalen Kooperationen zwischen 2008 und 2019 mehr als verdoppelt, von 251 auf 531.

Die Zunahme betrug im gleichen Zeitraum bei den Kunst- und Musikhochschulen 32\% (Anstieg von 253 auf 335 Kooperationen) und bei den kleinen Universitäten 20\% (Anstieg von 1.069 auf 1.814). Abb. 6.02

Die deutschen Hochschulen unterhielten im Jahr 2019 im Durchschnitt 0,4 internationale Hochschulkooperationen pro Professur. Bei der Auswertung nach Clustern lagen die kleinen Universitäten mit 0,8 internationalen Kooperationen pro Professur deutlich vorn, gefolgt von den mittelgroßen $(0,5)$ und den kleinen Universitäten $(0,4)$. Unter den Fachhochschulen wiesen die mittelgroßen Fachhochschulen die meisten internationalen Kooperationen pro Professur auf $(0,4)$, vor den kleinen $(0,3)$ und den großen Fachhochschulen $(0,3)$. Die Zahl der internationalen Kooperationen pro Professur war bei den Kunst- und Musikhochschulen mit 0,1 am geringsten. Abb. 6.03

\section{Abbildung > Internationale Kooperationen (ohne Erasmus) 6.03 pro Professur nach Cluster, 2019}

1,0

0,8

0,6

0,4

0,2
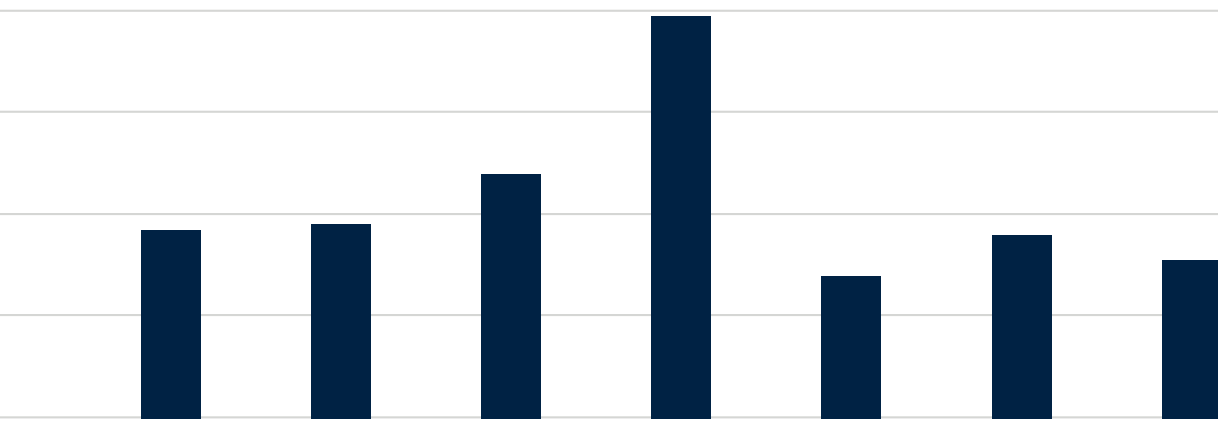

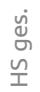

产

$\dot{\overrightarrow{\bar{E}}}$

$\frac{\dot{\vec{v}}}{\overline{\mathrm{s}}}$

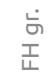

$\dot{\vec{E}}$
$\stackrel{T}{\leftarrow}$

$\underset{\dot{\underline{x}}}{\frac{\dot{T}}{\Psi}}$

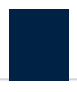

2019

- Internationale Kooperationen (ohne Erasmus) pro Professur

6 Die Gesamtzahlen beinhalten die auch geringe Anzahl der internationalen Kooperationen des Hochschulclusters „Sonstige Hochschulen“ (philosophisch-theologische Hochschulen sowie Verwaltungsfachhochschulen). 


\subsection{Verteilung der Hochschulkooperationen nach Ländern und Regionen}

Bei den internationalen Kooperationen nach Region liegen Ostasien und Nordamerika vorn (beide etwa 17\% aller Kooperationen), dicht gefolgt von Westeuropa (16,5\% aller Kooperationen). Zwischen 2008 und 2019 ist der Anteil der internationalen Kooperationen mit Partnern in Westeuropa stark gefallen (von 32,7\% auf 16,5\%); auch der Anteil der Kooperationen mit Partnern in Mittel- und Südosteuropa sowie in Osteuropa und Zentralasien hat abgenommen (von 14,3\% auf 7,5\% bzw. von 9,0\% auf 8,5\%). Eventuell zeigt sich hier der Einfluss der Förderung von strategischen Partnerschaften innerhalb Europas im Rahmen des Erasmus-Programms.

Am stärksten gestiegen ist der Anteil der Kooperationen mit Partnern in den Regionen Ostasien und Süd- und Mittelamerika; der Anteil der Region Ostasien stieg von 9,3\% auf $17,0 \%$ und der der Region Süd- und Mittelamerika von $7,4 \%$ auf $13,5 \%$ Abb. 6.04
Die Kooperationen mit den zehn wichtigsten Partnerländern umfassen knapp die Hälfte $(47,1 \%)$ aller Hochschulpartnerschaften außerhalb des ErasmusProgramms. Die meisten internationalen Hochschulkooperation bestanden 2019 mit Partnern in den USA, China und der Russischen Föderation. Bei acht der zehn häufigsten Partnerländer hat die Zahl der Kooperationen zwischen 2008 und 2019 zugenommen, am stärksten bei südkorea (knappe Verfünffachung von 108 auf 535 Kooperationen), China (mehr als Verdreifachung von 441 auf 1.402 Kooperationen) und Brasilien (gleichfalls etwa verdreifachung von 212 auf 639).

Gesunken ist die Zahl der Kooperationen dagegen mit Partnern in Großbritannien (Rückgang von 661 auf 475 Kooperationen) und in Frankreich (Rückgang von 635 auf 517) - wohlgemerkt außerhalb des ErasmusProgramms. Bemerkenswert ist auch die verhältnismäßig große Anzahl von Hochschulkooperationen mit Österreich außerhalb des Erasmus-Programms. Abb. 6.05 
Abbildung >> Verteilung der internationalen Kooperationen (ohne Erasmus)

6.04 nach Partnerregion im Zeitverlauf

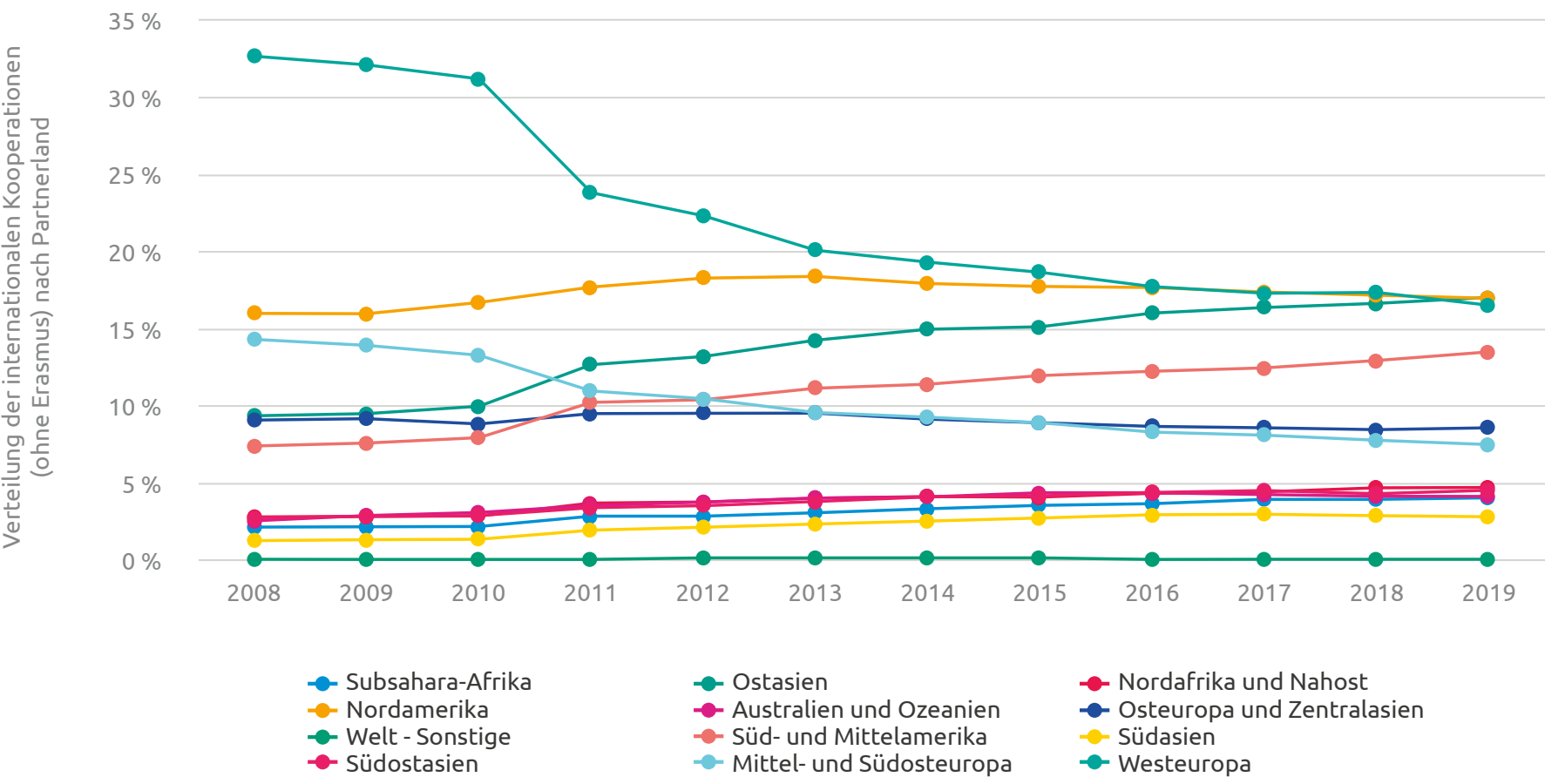

Siehe auch: >> Internationale Kooperationen (ohne Erasmus) nach Region der Partnerhochschule im Zeitverlauf

Abbildung >> Internationale Kooperationen (ohne Erasmus) nach Land/Region 6.05 der Partnerhochschule im Zeitverlauf

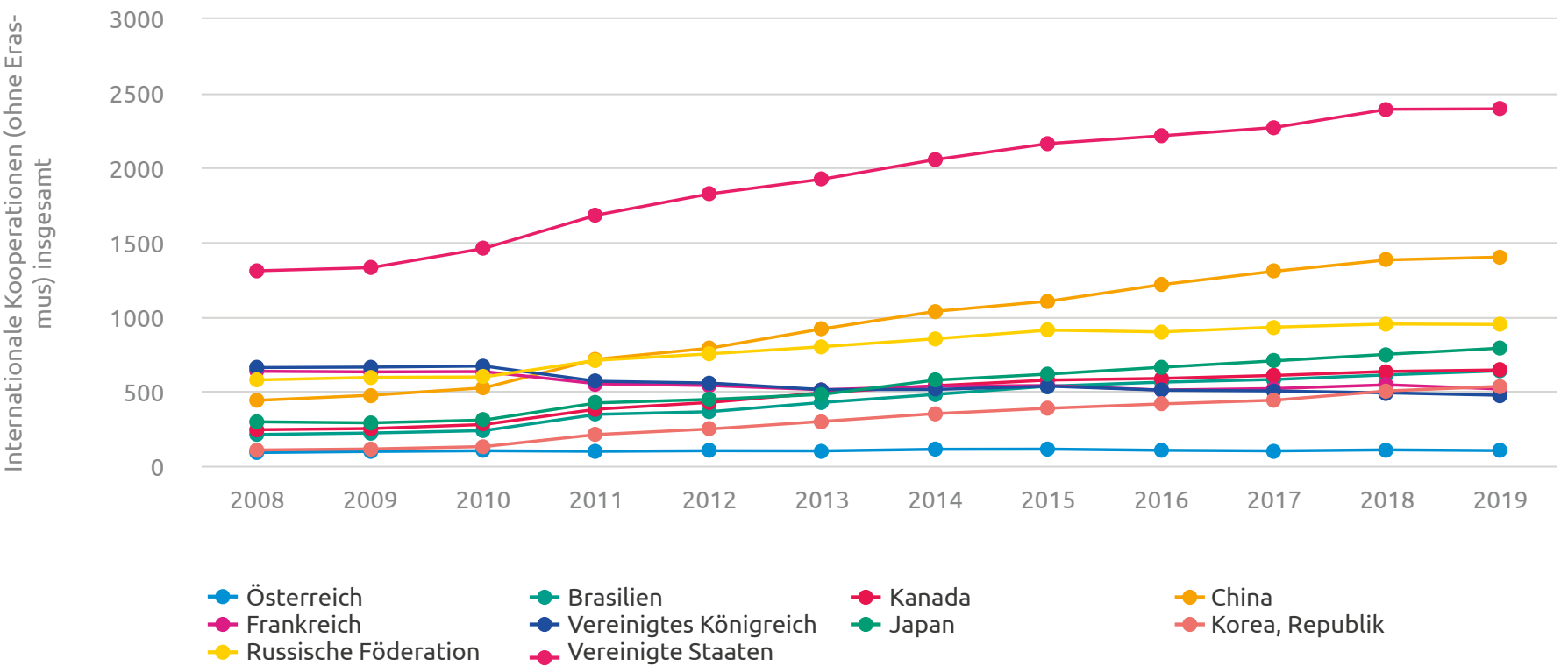

Siehe auch: >> Verteilung der internationalen Hochschulkooperationen (ohne Erasmus) nach Partnerland im Zeitverlauf 


\section{(10) Internationale Publikationen}

- Der Anteil der internationalen Ko-Publikationen steigt: 2018 hatten fast $\mathbf{4 7 \%}$ der Publikationen von Autorinnen und Autoren an Forschungseinrichtungen in Deutschland mindestens eine internationale Ko-Autorin oder einen internationalen Ko-Autor.

- Knapp die Hälfte der 2018 veröffentlichten internationalen KoPublikationen hatte einen Bezug zur Fächergruppe Mathematik/ Naturwissenschaften, etwas über ein Viertel zu Humanmedizin/ Gesundheitswissenschaften und knapp ein Fünftel zu den Ingenieurswissenschaften.

- Deutsche Hochschulen publizierten 2018 am häufigsten mit Autorinnen und Autoren aus den USA, Großbritannien und der Schweiz. China und Australien sind zunehmend wichtig. 


\section{Internationale Publikationen}

Daten zu Publikationen und internationalen Ko-Publikationen der Hochschulen stellen einen wichtigen Indikator zur Messung der Internationalität der Forschung dar. Sie ergänzen forschungsrelevante Daten wie DFG-Projekte an Hochschulen mit internationaler Beteiligung, Drittmittel der EU und Förderung der internationalen Mobilität von Forschenden durch die Alexander-von-Humboldt-Stiftung und den DAAD.

Die im HSI-Monitor verwendeten Daten wurden mit Hilfe der > > Datenbank Dimensions (Anbieter: Digital Science) berechnet. Die Datenbank wurde als Berechnungsgrundlage ausgewähtt, da eine gute Erfassung auch der Publikationen von Fachhochschulen und kleineren Hochschulen möglich ist, wodurch die unterschiedliche Schwerpunktsetzung der verschiedenen Hochschulen berücksichtigt werden kann. Die Auswahl der berücksichtigten Journals und Publikationen durch den Anbieter kann zu Abweichungen bei den aus- gewiesenen Zahlen der Publikationen und internationalen Ko-Publikationen im Vergleich zu anderen bibliometrischen Veröffentlichungen führen.

Im Jahr 2018 konnten für knapp drei Viertel der im Datensatz berücksichtigten 434 Hochschulen Publikationen nachgewiesen werden. Bei den übrigen Hochschulen ohne nachgewiesene Publikationen handelt es sich in erster Linie um kleine, private Fachhochschulen, Verwaltungsfachhochschule sowie Philosophisch-Theologische Hochschulen, bei denen von einem geringen Publikationsoutput ausgegangen werden kann.

\section{Lesen Sie mehr zum Thema unter: > HSI-Monitor > Themen > Internationale Publikationen}




\subsection{Grunddaten}

Die Anzahl der Publikationen insgesamt wie auch die Anzahl der internationalen Ko-Publikationen - also Publikationen mit mindestens einer internationalen Ko-Autorin oder einem internationalen Ko-Autor einer Forschungsstelle im Ausland - nehmen stark zu. Zwischen 2014 und 2018 ist die Gesamtzahl der Publikationen um gut ein Viertel von 123.211 auf 154.655 Publikationen gewachsen; die Zahl der internationalen Ko-Publikationen stieg im gleichen Zeitraum sogar um ca. 34 \% von 54.287 auf 72.585. Abb. 7.01

Die starke Zunahme der Zahl der internationalen KoPublikationen spiegelt sich im Anteil der internationalen Ko-Publikationen an allen Publikationen wider, dieser ist im Durschnitt der Hochschulen zwischen 2014 und 2018 von 44,1\% auf 46,9\% gestiegen. Den höchsten Anteil an internationalen Ko-Publikationen wiesen 2018 die großen Universitäten auf (48,9\%), gefolgt von den mittelgroßen $(46,6 \%)$ und den kleinen Universitäten (41,6\%). Bei den Fachhochschulen lag der Anteil bei durchschnittlich 28,9\%, wobei die mittelgroßen Fachhochschulen mit 29,5\% etwas vor den kleinen und den großen Fachhochschulen lagen (beide 28,5\%). A Abb. $\mathbf{7 . 0 2}$

Fast 96\% aller Publikationen und 97,5\% aller internationalen Ko-Publikationen entstanden im Jahr 2018 mit Beteiligung von Universitäten. Obwohl der Anteil der Fachhochschulen an beiden Kategorien sehr gering ist, ist er jedoch in den letzten Jahren gewachsen: während Fachhochschulen im Jahr 2014 nur 2,6\% aller Publikationen und 0,8\% der internationalen Ko-Publikationen stellten, wurden 2018 bereits 3,9\% aller Publikationen und 1,2\% der internationalen Ko-Publikationen mit Autorinnen und Autoren von Fachhochschulen veröffentlicht. Grundsätzlich publizieren Wissenschaftlerinnen und Wissenschaftler an Fachhochschulen eher mit Ko-Autorinnen oder KoAutoren als allein.

Die Betrachtung der internationalen Ko-Publikationen im Verhältnis zur Anzahl der Professorinnen und Professoren ermöglicht eine Bewertung der Publikationstätigkeit unter Berücksichtigung der personellen Ausstattung der Hochschulen. Zu beachten ist allerdings, dass große Unterschiede hinsichtlich der Rahmenbedingungen und Missionen der verschiedenen Hochschultypen existieren, zum Beispiel in Bezug auf die Zeit, die für Forschungsund Publikationstätigkeiten eingesetzt werden kann. So müssen Professorinnen und Professoren an Fachhochschulen einen größeren Teil ihrer Arbeitszeit als ihre Kolleginnen und Kollegen an Universitäten für die Lehre aufwenden. Auch an den Kunst- und Musikhochschulen kommt der Lehre ein größerer Stellenwert zu als der Forschung.

\section{Im Detail:}

>> Internationale Ko-Publikationen pro 100 Professuren, 2018 
Abbildung > Anzahl der Publikationen und der internationalen Ko-Publikationen, 2014-2018 7.01

$180 \mathrm{k}$

$180 \mathrm{k}$

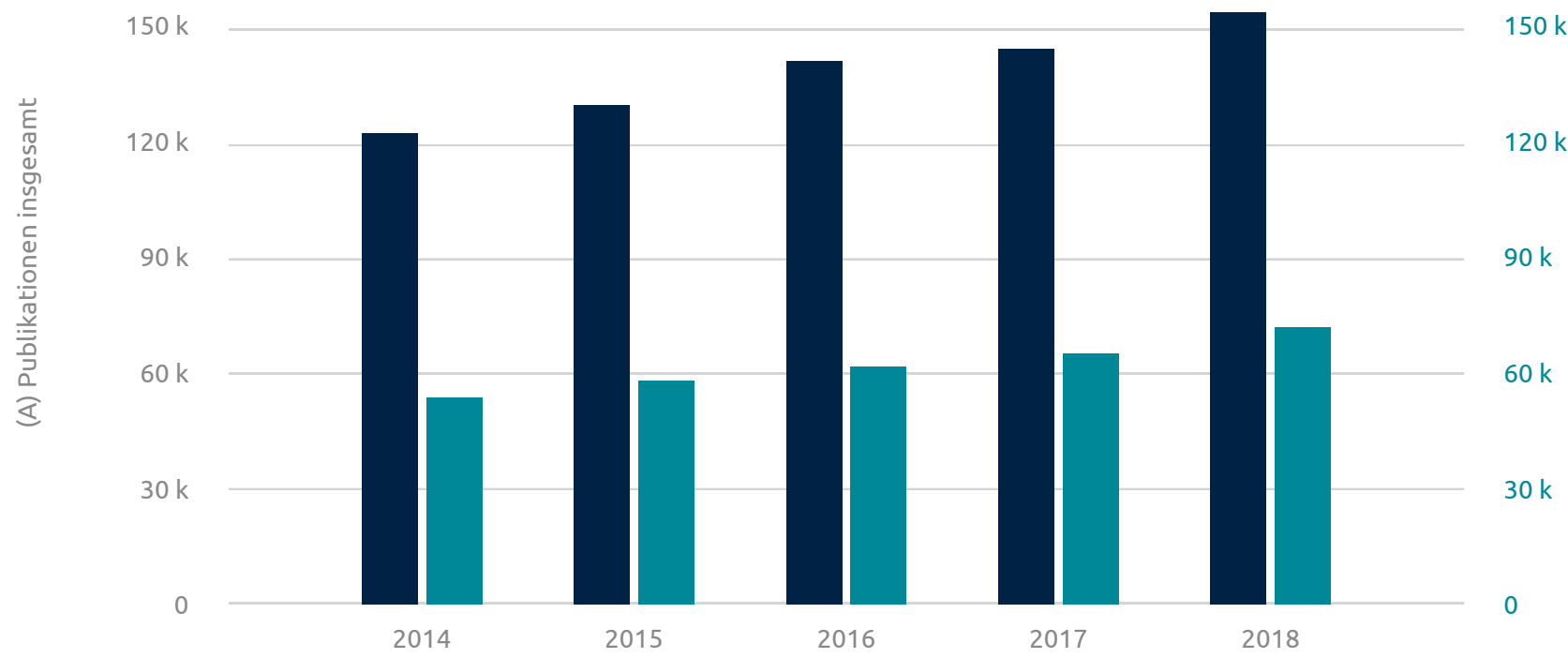

- (A) Publikationen insgesamt

- (B) Internationale Ko-Publikationen

Abbildung > Anteil der internationalen Ko-Publikationen an allen Publikationen nach Cluster, 2018 7.02

$60 \%$

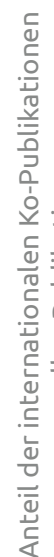

$50 \%$

$40 \%$

$30 \%$

$20 \%$

$10 \%$

$0 \%$
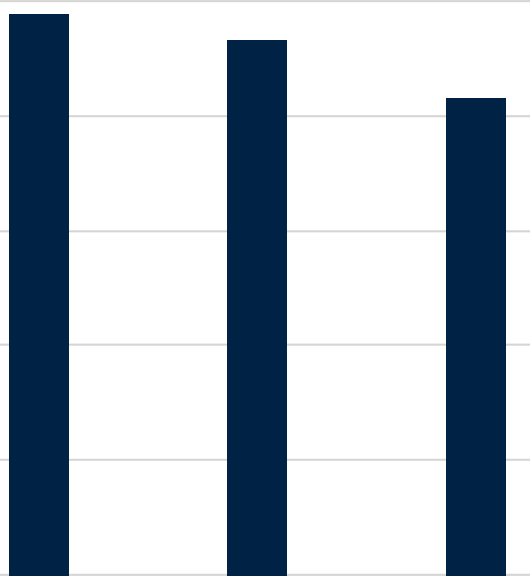

高

$\underset{\dot{v}}{\dot{\vec{c}}}$
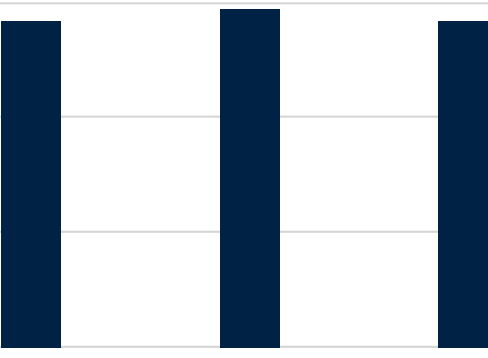

$\dot{\vec{E}}$

宊

$\dot{\bar{E}}$
$\frac{T}{4}$

$\underset{\text { 妾 }}{\stackrel{\dot{T}}{\amalg}}$

2018

- Anteil der internationalen Ko-Publikationen an allen Publikationen 
Abbildung

>> Verteilung der internationalen Ko-Publikationen nach Fächergruppe, 2014-2018

7.03

$60 \%$

$50 \%$

$40 \%$

$30 \%$

$20 \%$

$10 \%$

$0 \%$
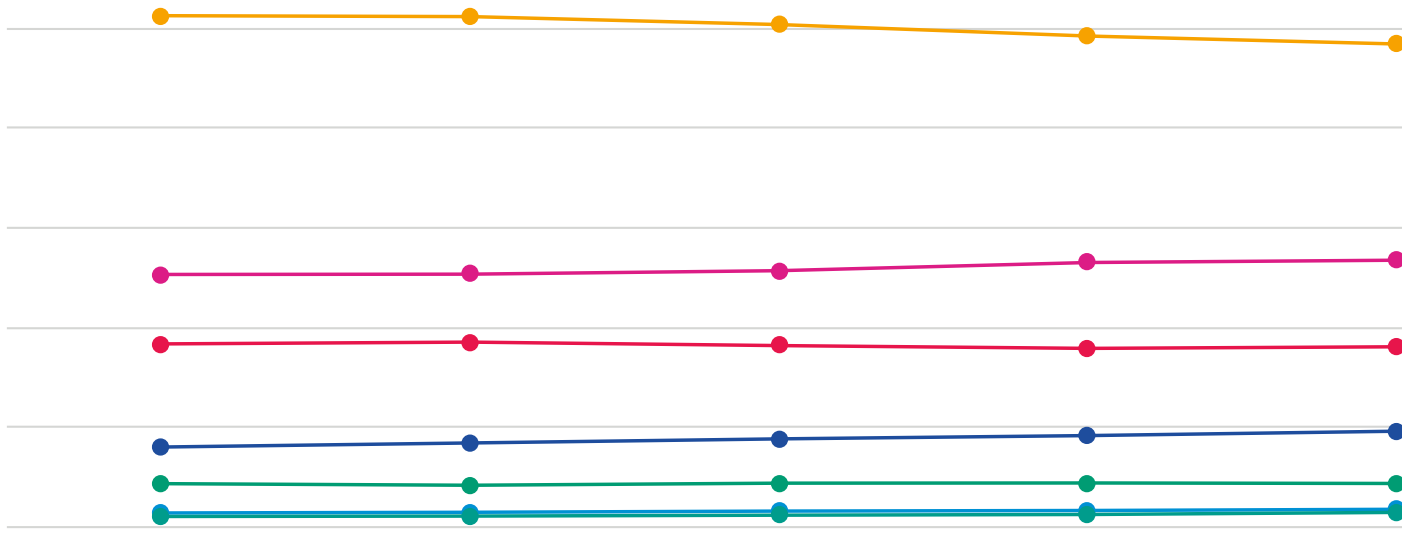

2014

2015

2016

2017

2018

Agrar-, Forst- und Ernährungswissenschaften, Veterinärmedizin

- Geisteswissenschaften

- Ingenieurwissenschaften

- Mathematik, Naturwissenschaften

- Humanmedizin/Gesundheitswissenschaften
Rechts-, Wirtschafts- und Sozialwissenschaften Außerhalb der Studienbereichsgliederung/Sonstige Fächer

Anmerkung: Die Fächergruppen Sport

sowie Kunst, Kunstwissenschaft sind aufgrund geringer Publikationszahlen ausgeblendet.

Siehe auch: >> Internationale Ko-Publikationen nach Fächergruppe, 2014-2018

Abbildung >> Verteilung der internationalen Ko-Publikationen nach Partnerland, 2014-2018 7.04
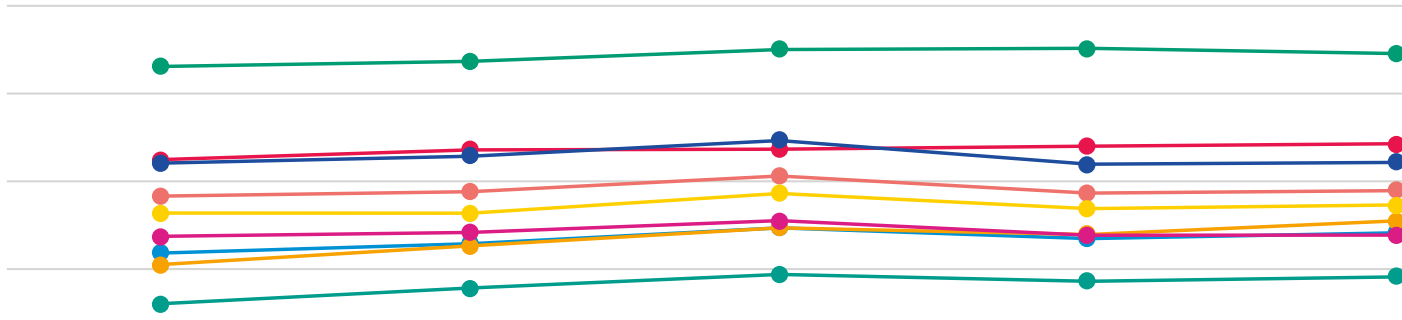

$\%$

Österreich

Spanien

- Niederlande
Schweiz

Vereinigtes Königreich - $\quad$ Italien 


\subsection{Verteilung der internationalen Ko-Publikationen nach Fächergruppe und Partnerland}

Im aktuellen Bericht erfolgt, in Angleichung an die anderen Themenbereiche, die Zuordnung der Publikationen zu Fächergruppen auf der Basis der Systematik des Statistischen Bundesamtes (Destatis). ${ }^{7}$

Knapp die Hälfte der 2018 veröffentlichten internationalen Ko-Publikationen (48,3\%) entstanden in der Fächergruppe Mathematik/Naturwissenschaften, etwas über ein Viertel (26,7\%) in der Humanmedizin/Gesundheitswissenschaften, 18,0\% in den Ingenieurwissenschaften. Mit dem Anstieg der Publikationszahl zwischen 2014 und 2018 ging nur eine geringfügige Änderung der Anteile der einzelnen Disziplinen einher: Der Anteil der Publikationen mit Bezug zu Mathematik/Naturwissenschaften ist zwischen 2014 und 2018 beständig von 51,2\% auf 48,3\% gesunken, der Anteil der Publikationen mit Relevanz zu Rechts-, Wirtschafts- und Sozialwissenschaften und zu Humanmedizin/Gesundheitswissenschaften ist geringfügig gestiegen (um 1,6 Prozentpunkt bzw. 1,4 Prozentpunkte). A Abb. $\mathbf{7 . 0 3}$
Die wichtigsten Partnerländer für internationale Ko-Publikationen waren im Jahr 2018 die USA, Großbritannien sowie die Schweiz. 31,5\% aller internationale Ko-Publikationen hatten mindestens eine Ko-Autorin oder einen Ko-Autor mit einer US-amerikanischen Affiliierung, 22,2\% mit einer britischen und 17,1\% mit einer schweizerischen. ${ }^{8}$ Außer den USA waren China (siebtwichtigstes Partnerland, Ko-Autorenschaft bei 12,7\% der Publikationen) und Australien (zehntwichtigstes Partnerland, Ko-Autorenschaft bei 9,5\%) die einzigen nicht-europäischen Partnerländer unter den Top 10. Der Anteil von internationalen Ko-Publikationen mit chinesischen oder australischen Ko- Autorinnen und -Autoren ist zwischen 2014 und 2018 um 2,5 Prozentpunkte bzw. 1, 5 Prozentpunkte gestiegen. Abb. 7.04 Bezogen auf die Anteile in 2014 bedeutet dies einen deutlichen Anstieg um 24\% bzw. 18,7\%.

7 Die bibliometrische Datenbank Dimensions nimmt eine Zuordnung von Publikationen zu dem Klassifikationssystem „Fields of Research (FOR)“ vor (Mehr lesen: >> Fields of Research - Dimension). Um die FOR-Zuordnung auf Destatis-Fächergruppen abzubilden, wurde eine Umsetzungssystematik entwickelt. Bei Publikationen mit Relevanz zu mehreren Fächergruppen erfolgt eine Mehrfachzuordnung, das heißt dass Publikationen unter Umständen mehrfach berücksichtigt werden. Die Gesamtzahl der Zuordnungen zu Fächergruppen kann dementsprechend größer als die Gesamtzahl der Publikationen sein, und die Summe aller Prozentangaben kann 100\% übersteigen. Die Fächergruppe „Außerhalb der Studienbereichsgliederung / Sonstige Fächer" umfasst vorrangig Publikationen, die keiner Fächergruppe eindeutig zugeordnet werden können.

8 Wie bei den Fächergruppen erfolgt bei der Auswertung nach Partnerländern eine Mehrfachzuordnung, das heißt dass Publikationen mit mehreren Ko-Autorinnen und -Autoren aus unterschiedlichen Ländern unter Umständen mehrfach berücksichtigt werden. Die Gesamtzahl der Zuordnungen zu Ländern kann dementsprechend größer als die Gesamtzahl der Publikationen sein, und die Summe aller Prozentangaben kann $100 \%$ übersteigen. 


\section{(- Förderungen des DAAD}

- Die Anteile der DAAD-Individualgeförderten aus dem Ausland und aus Deutschland sind bei den Universitäten und den Kunstund Musikhochschulen deutlich höher als bei den Fachhochschulen.

- Die populärsten Zielländer für Geförderte im PROMOS-Programm waren im Jahr 2018 vier außereuropäische Länder: die USA, China, Australien und Kanada.

- Mittelgroße Universitäten und Fachhochschulen werben die höchsten Förderbeträge pro Studentin und Student im Bereich der Projekt-/ Programmförderung (ohne EU-Mittel) ein; bei der Förderung aus EU-Mitteln führen die kleineren Institutionen. 


\section{Förderungen des DAAD}

Der Deutsche Akademische Austauschdienst (DAAD) fördert die internationalen akademischen Beziehungen sowie die Internationalisierung der Hochschulen. Die Umsetzung erfolgt im Rahmen von mehr als 250 Förderprogrammen, die überwiegend aus Mitteln des Bundes finanziert werden. Der Fokus liegt auf der Förderung von Mobilität, entweder über die direkte Individualförderung oder durch Finanzierung entsprechender Projekte und Programme an den Hochschulen.

Lesen Sie mehr zum Thema unter: >> HSI-Monitor > Themen > Förderungen des DAAD

\subsection{Individualförderung}

Zwischen 2014 und 2017 ist die Gesamtzahl der DAADIndividualgeförderten von 18.912 auf 16.217 zurückgegangen. Die Zahl ist jedoch bis 2018 wieder auf 17.642 gestiegen, was eine Zunahme um 8,8\% im Vergleich zum Vorjahr darstellt. ${ }^{2}$ Die Ursachen der zeitlichen Abweichungen sind vielfältig, insbesondere spielen Änderungen des Programmangebotes, der Mittellage und der Nachfrage eine Rolle.

Im Detail:

> Gesamtzahl der DAAD-Individualgeförderten, 2014-2018

Auch die Zahlen der Individualgeförderten, nach Geförderten aus dem Ausland und aus Deutschland getrennt betrachtet, unterlagen ähnlichen Schwankungen. Sie nahmen zwischen 2014 und 2017 von 12.303 auf 10.578 bzw. von 6.609 auf 5.639 ab. Sie stiegen jedoch bis 2018 wieder an, bei Individualgeförderten aus dem Ausland auf 11.013 (Zunahme um 4,1\% im Vergleich zum Vorjahr) und bei Individualgeförderten aus Deutschland auf 6.629 (Zunahme um 17,6\%). A Abb. 8.01

Abbildung > DAAD-Individualgeförderte aus dem Ausland und aus Deutschland, 2014-2018 8.01

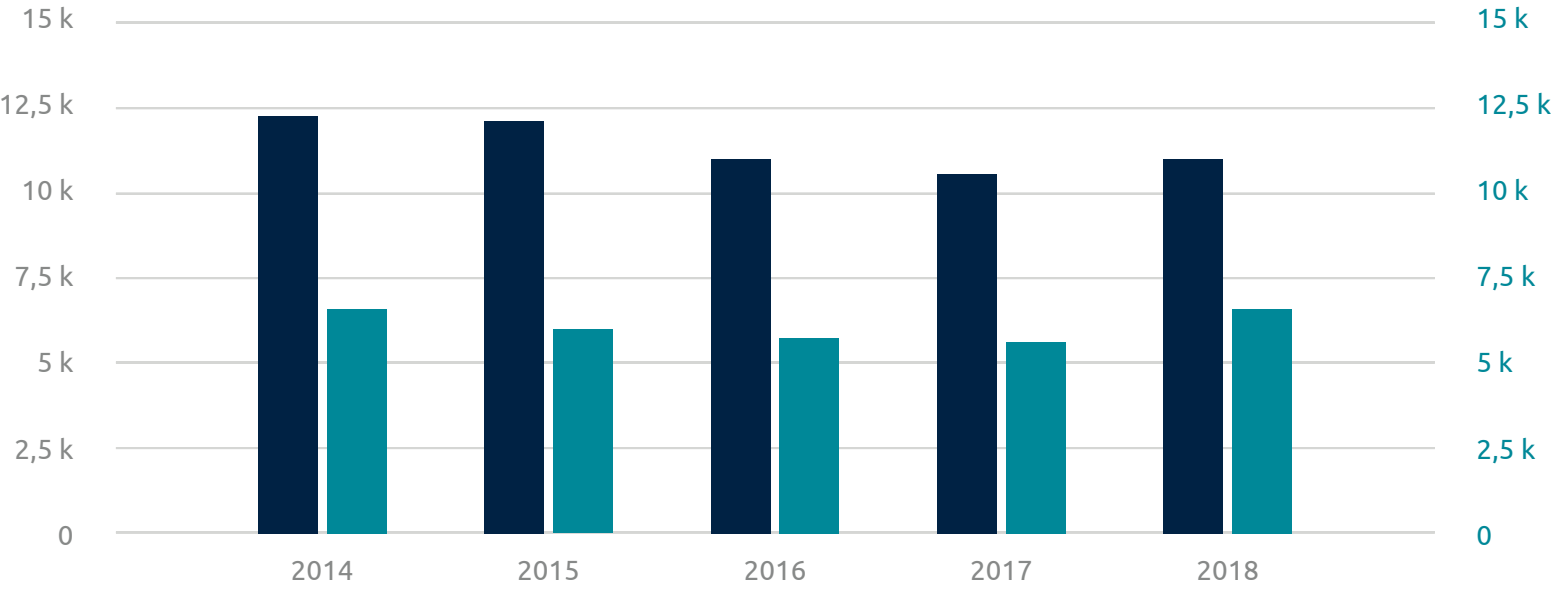

- (A) Individualgeförderte aus dem Ausland

- (B) Individualgeförderte aus Deutschland 
Bei der Unterteilung nach Cluster wiesen die Universitäten und die Kunst- und Musikhochschulen die höchsten Anteile an Stipendiatinnen und Stipendiaten aus dem Ausland auf (Universitäten: 0,5\%, Kunst- und Musikhochschulen: 0,5\%). Die Fachhochschulen lagen mit einem Anteil von 0,1\% deutlich unter dem Gesamtdurchschnitt der Hochschulen. Abb. 8.02

Bei den Individualgeförderten aus Deutschland wiesen die kleineren Universitäten den höchsten Anteil von Geförderten an der Gesamtzahl der Studierenden auf $(0,4 \%)$, gefolgt von den Kunst- und Musikhochschulen $(0,4 \%)$, den mittelgroßen $(0,3 \%)$ und den großen Universitäten (0,3\%). Auch hier liegen die Fachhochschulen deutlich unter dem Gesamtdurchschnitt der Hochschulen mit einem Anteil von 0,1\%.

A Abb. 8.03
Im aktuellen Berichtsjahr 2018 wurden durchschnittlich 34,70 Euro pro Studierender und Studierendem für Individualförderungen verausgabt. Die höchsten Förderbeträge pro Studentin und Student fanden sich bei den Kunst- und Musikhochschulen (61,67 Euro), gefolgt von den Universitäten (47,60 Euro). Die Fördermittel für Individualgeförderte an Fachhochschulen waren mit durchschnittlich 7,65 Euro deutlich geringer.

Abb. 8.04

\section{Abbildung >> Anteil der DAAD-Individualgeförderten aus dem Ausland an allen Studierenden 8.02 nach Cluster, 2018}

$0,6 \%$

$0,5 \%$

$0,4 \%$

$0,3 \%$

$0,2 \%$

$0,1 \%$

$0 \%$
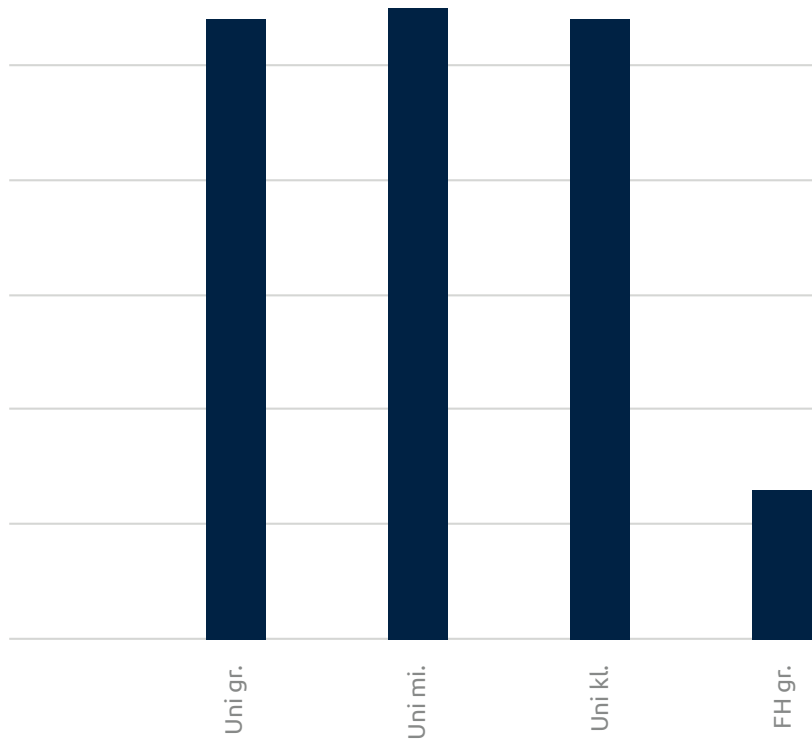

$\dot{\bar{\varepsilon}} \quad \frac{\dot{\vec{v}}}{\overline{\mathrm{s}}}$

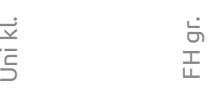

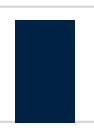

$\dot{\bar{E}}$
$\dot{I}$

2018 
Abbildung

8.03

$0,4 \%$

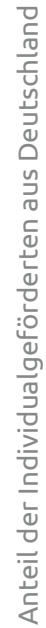

$0,35 \%$

$0,3 \%$

$0,25 \%$

$0,2 \%$

$0,15 \%$

$0,1 \%$

$0,05 \%$

$0 \%$

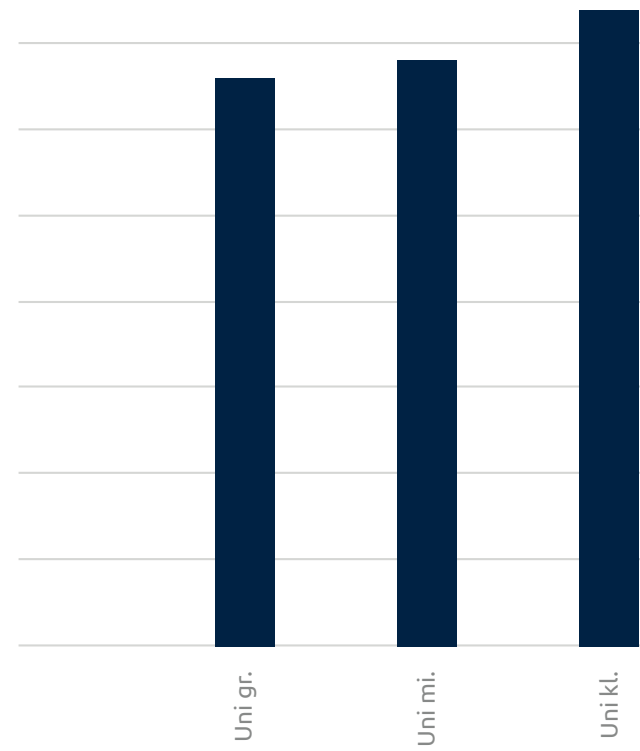

- Anteil der Individualgeförderten aus Deutschland an allen Studierenden >> Anteil der DAAD-Individualgeförderten aus Deutschland an allen Studierenden nach Cluster, 2018

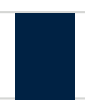

$\stackrel{\text { 殅 }}{\text { I }}$

2018

Abbildung >> DAAD-Fördermittel für Individualförderung pro Studentin und Student 8.04 nach Cluster, 2018 (Mittelwert in Euro)

70

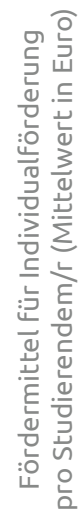

60

50

40

30

20

10

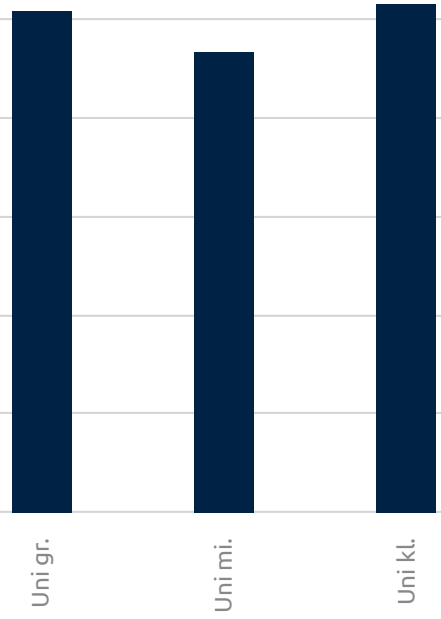

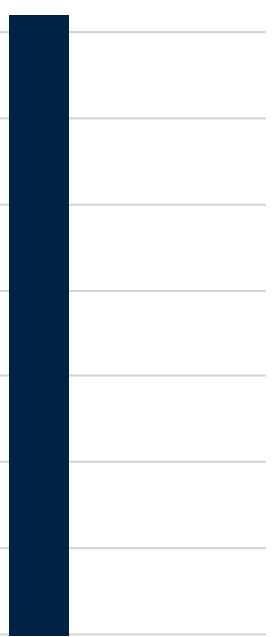

$\underset{\underline{I}}{I}$ 


\subsection{Geförderte im Rahmen des Programmes „PROMOS - Förderung der Mobilität von Studierenden und Promovierenden deutscher Hochschulen"}

Im Zuge der Entwicklung des HSI-Monitors wurden

Daten zum Hochschul-Programm „PROMOS - Programm zur Steigerung der Mobilität von Studierenden deutscher Hochschulen" in die Indikatorik aufgenommen. Diese Daten ermöglichen eine nuancierte Auswertung der Auslandsmobilität von Studierenden an deutschen Hochschulen nach Fächergruppe und Zielland. Im PROMOS-Programm entscheiden die Hochschulen selbst über den Einsatz der eingeworbenen Mittel, die Mobilitäten der Geförderten spiegeln daher sowohl die strategische Schwerpunktsetzung bei der Ausgestaltung der Angebote durch die Hochschulen als auch die Interessen der Studierenden wider.

Die Gesamtzahl der Geförderten im Promos-Programm ist im Gesamtzeitraum zwischen 2010 und 2018 um $165 \%$ von 4.943 auf 13.095 jährlich gestiegen.

Im Detail:

> Promos-Geförderte insgesamt, im Zeitverlauf
Die Zahl der weiblichen Promos-Geförderten lag bislang durchgehend über der Zahl der männlichen Geförderten, 2018 gab es ungefähr 1,4-mal mehr Stipendiatinnen als Stipendiaten (7.727 gegenüber 5.368). A Abb. 8.05

Die meisten Promos-Geförderten stammten 2018 aus der Fächergruppe der Rechts-, Wirtschafts- und Sozialwissenschaften (5.110), gefolgt von den Geisteswissenschaften (2.366) und den Ingenieurswissenschaten (2.293).

- Abb. 8.06

Der größte Teil der Promos-Geförderten nahm 2018 an einem Mobilitätsprogramm in die USA teil (2.036), gefolgt von China (702), Australien (656) und Kanada (581). Den größten Zuwachs gab es zwischen 2017 und 2018 bei den Zielländern Österreich (Zunahme um 16,8\%), Spanien (16,8\%) sowie Italien (14,8\%). Abb. $\mathbf{8 . 0 7}$

\section{Abbildung >> Promos-Geförderte nach Geschlecht im Zeitverlauf} 8.05

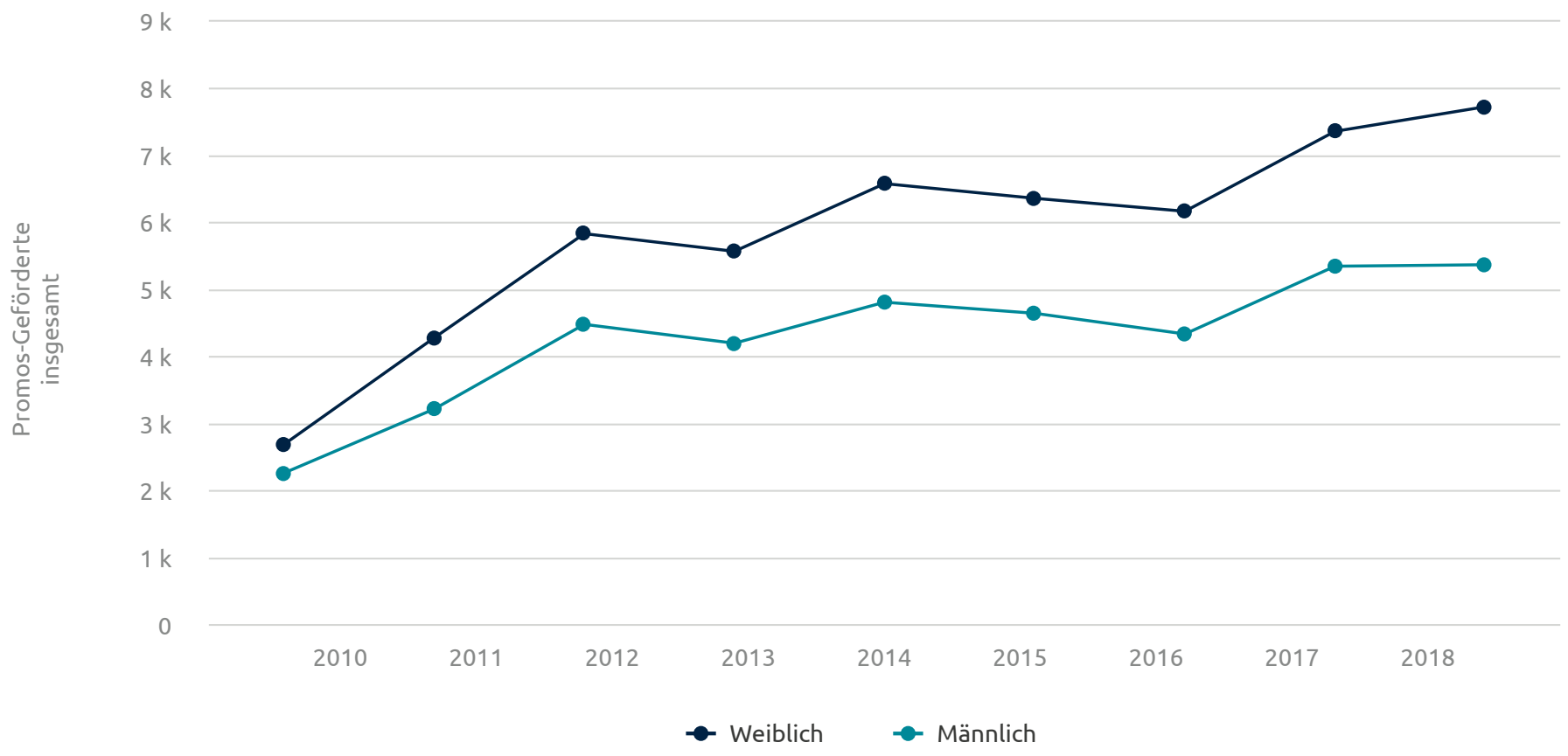

Siehe auch: >> Verteilung der Promos-Geförderten nach Geschlecht im Zeitverlauf 


\section{Abbildung >> Promos-Geförderte nach Fächergruppe, 2018}

8.06

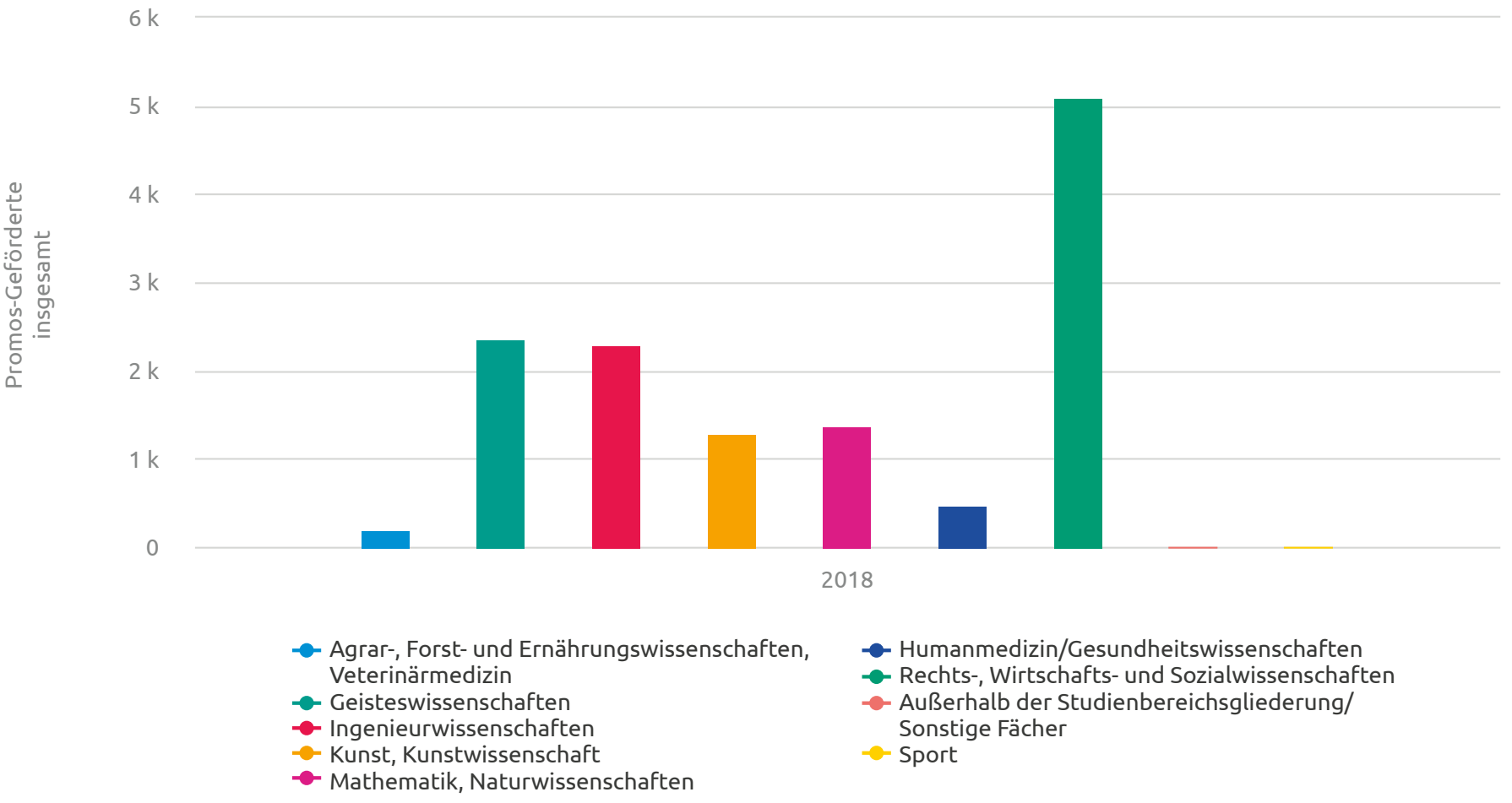

Abbildung >> Promos-Geförderte: Top 10 Zielländer, 2018

8.07

2500

2000

1500

1000

500
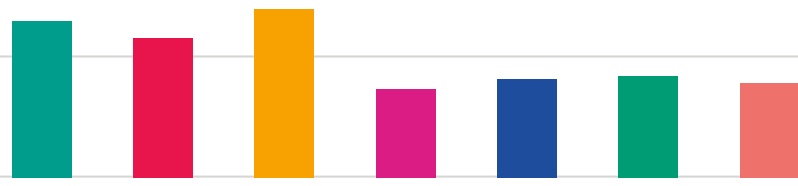

2018

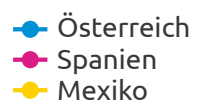

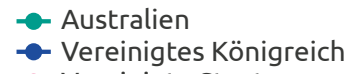

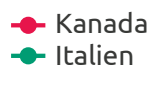

- China

- Vereinigte Staaten
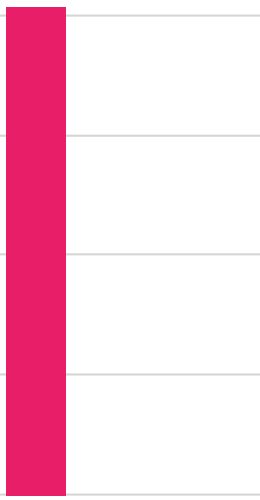

- Mexiko

Siehe auch: >> Promos-Geförderte: Top 10 Zielländer, im Zeitverlauf 


\subsection{Förderung für Projekte und Programme}

Bei den Fördermitteln für Projekte und Programme (ohne Förderung aus EU-Mitteln) lagen im Jahr 2018 die Universitäten mit 59,19 Euro pro Studentin und Student an der Spitze; die Kunst- und Musikhochschulen folgten mit 49,03 Euro. Die Fachhochschulen verzeichneten hier die niedrigsten Fördersummen pro Studentin und Student, der Unterschied zu den anderen Clustern ist aber deutlich weniger ausgeprägt als bei den Individualgeförderten (siehe Abschnitt 8.1). Innerhalb der Cluster zeigen sich deutliche Differenzen: Die mittelgroßen Universitäten liegen mit 69,47 Euro vor den kleinen und den großen Universitäten (65,71 Euro bzw. 57,90 Euro), und auch die mittelgroßen Fachhochschulen weisen mit 44,20 Euro höhere Förderbeträge auf als die großen und die kleinen Fachhochschulen
(28,68 Euro bzw. 19,65 Euro). Der Trend, dass die Institutionen mittlerer Größe führend sind, lässt sich bei Universitäten seit 2016 beobachten, bei Fachhochschulen bereits seit 2009. Abb. $\mathbf{8 . 0 8}$

Bei der Förderung aus EU-Mitteln für Projekte und Programme sind die Unterschiede zwischen den verschiedenen Hochschultypen geringer und auch die Verteilung zwischen den Clustern gestaltet sich anders. Für Universitäten und Fachhochschulen gilt, dass der EU-Förderbetrag pro Studentin und Student mit abnehmender Hochschulgröße zunimmt. Dieser Zusammenhang besteht bei Universitäten seit 2012 und bei Fachhochschulen seit 2013. Abb. 8.09

\section{Abbildung > DAAD-Fördermittel für Projekte und Programme (ohne EU-Förderung) pro Studentin 8.08 und Student nach Cluster, 2018 (Mittelwert in Euro)}

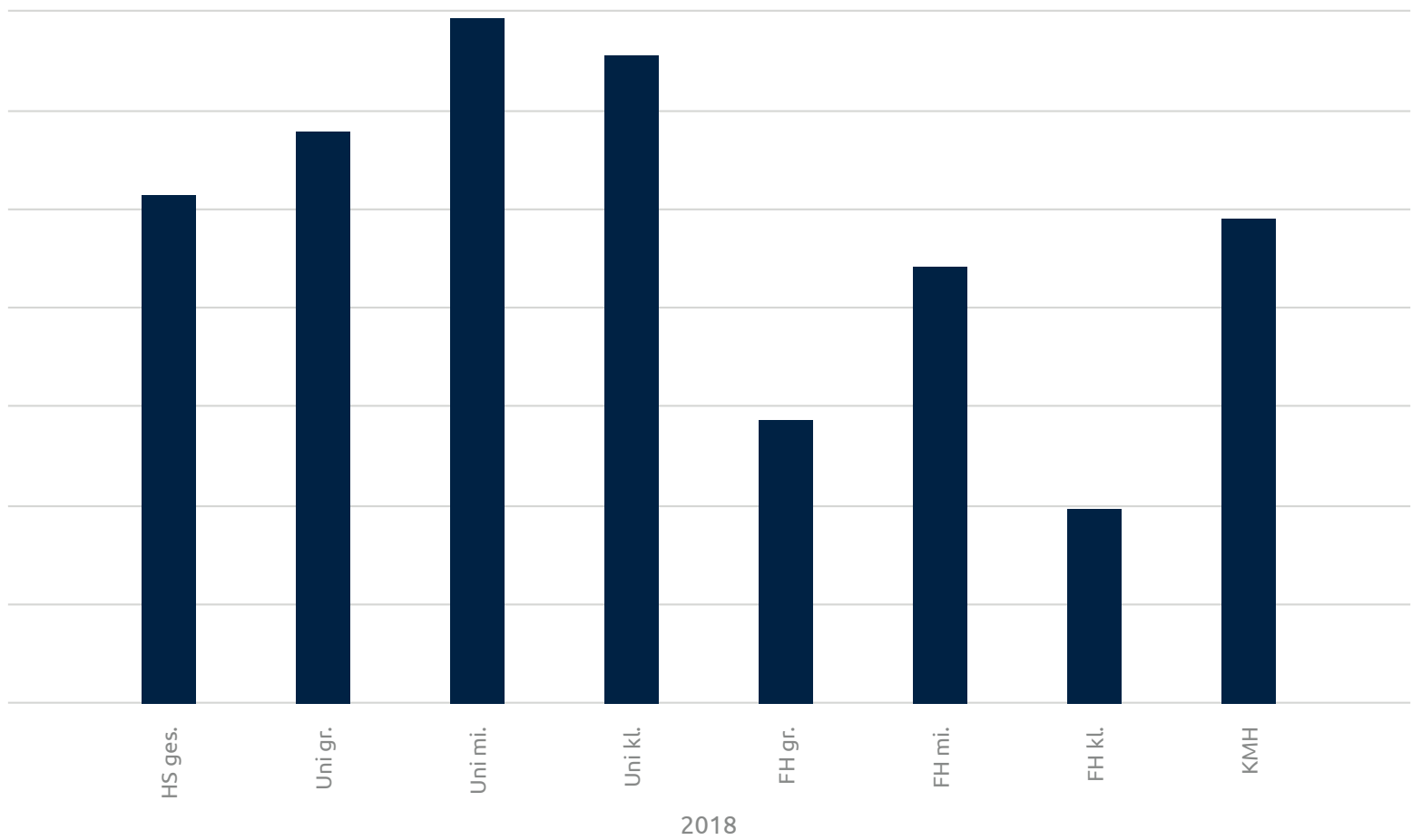

- Fördermittel für Projekte und Programme (ohne EU-Förderung) pro Studierendem/r (Mittelwert in Euro) 
Abbildung > DAAD-Fördermittel für Projekte und Programme (nur EU-Förderung) pro Studentin 8.09 und Student nach Cluster, 2018 (Mittelwert in Euro)

60

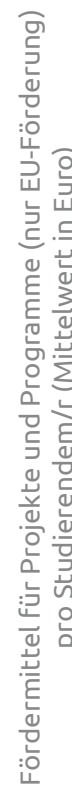

50

40

30

20

10

0

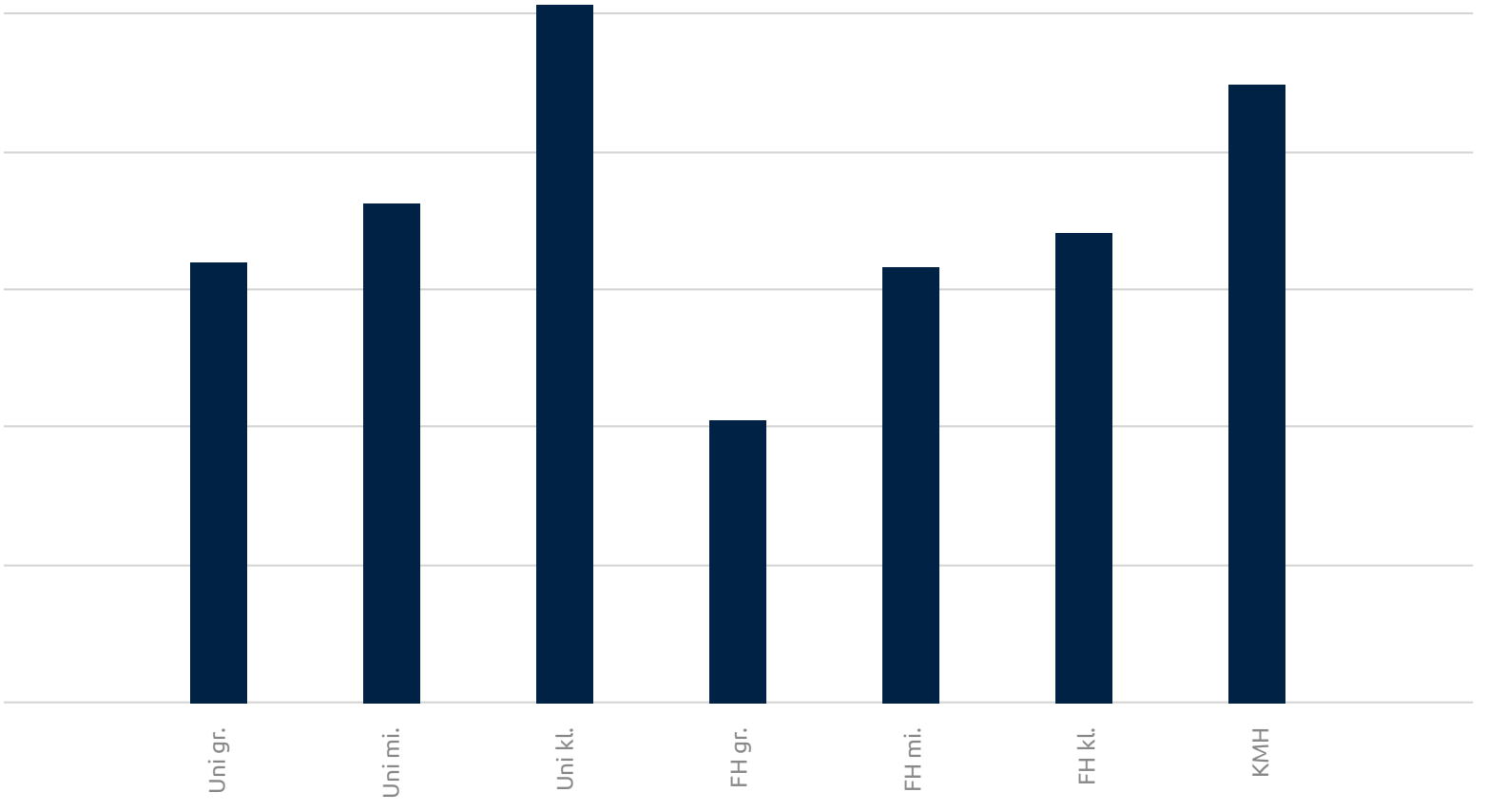

2018

- Fördermittel für Projekte und Programme (nur EU-Förderung) pro Studierendem/r (Mittelwert in Euro) 


\section{(B) Forschungsförderung der Alexander von Humboldt-Stiftung}

- Während die Zahl der Aufenthalte von Stipendiatinnen und Stipendiaten sowie Preisträgerinnen und -trägern der Alexander von Humboldt-Stiftung an großen Universitäten im Beobachtungszeitraum um fast $70 \%$ zugenommen hat, ist die Zahl an mittelgroßen und kleinen Hochschulen stark zurückgegangen.

- Etwa die Hälfte aller Aufenthalte entfallen auf die Fächergruppe Mathematik/Naturwissenschaften, ein Viertel auf die Geisteswissenschaften.

- Die USA sind das wichtigste Herkunftsland. Auf dem zweiten Platz folgt mit deutlichem Abstand China, Indien liegt auf dem dritten Platz. 


\section{Forschungsförderung der Alexander von Humboldt-Stiftung}

Die Alexander von Humboldt-Stiftung (AvH) fördert Wissenschaftskooperationen zwischen exzellenten Forscherinnen und Forschern aus dem Ausland und Deutschland. Die Stiftung pflegt ein Netzwerk von weltweit über 30.000 Alumnae und Alumni aller Fachgebiete in über 140 Ländern.

Die Forschungsaufenthalte ausländischer HumboldtGeförderter an deutschen Gastinstitutionen werden auf der Internetseite der Stiftung im sogenannten "Humboldt-Ranking" dargestellt. (Mehr lesen:

>> Humboldt-Ranking) Diese Statistik bietet einen wichtigen vergleichenden Indikator für die internationalen Forschungskooperationen deutscher Forschungseinrichtungen.

Die Daten zu den AvH-Geförderten werden im HSI-Monitor in Fünfjahreszeiträumen (5 Jz | „2017“ = 2013-2017) zusammengefasst, die Bezugsjahre der Diagramme beziehen sich dabei immer auf das letzte Jahr im Zeitraum.

\section{Lesen Sie mehr zum Thema unter:}

>> HSI-Monitor > Themen > Förderungen der AvH

\subsection{Grunddaten}

Im aktuellen Fünfjahreszeitraum 2014-2018 wurden durch die Alexander von Humboldt-Stiftung insgesamt 4.494 Forschungsaufenthalte ausländischer Spitzenwissenschaftlerinnen und -wissenschaftler an deutschen Hochschulen gefördert. Die Zahl der Aufenthalte pro Fünfjahreszeitraum ist seit 2009 innerhalb einer engen Bandbreite zwischen 4.454 und 4.661 weitgehend konstant geblieben. Abb. 9.01

Betrachtet man die Anzahl der Humboldt-Aufenthalte pro 100 C4/W3-Professuren, zeigt sich, dass diese im Laufe des Beobachtungszeitraums von 33,8 auf 29,8 gefallen sind. Hierfür ist allerdings weniger der leichte
Rückgang bei den Aufenthalten von ausländischen AvH-Gastwissenschaftlerinnen und -wissenschaftlern verantwortlich als vielmehr der Zuwachs bei der Zahl der C4/W3-Professuren an deutschen Universitäten zwischen 2008 und 2018 haben diese von 12.882 auf 15.096 Personen zugenommen.

Im Detail:

>> Aufenthalte von AvH-Geförderten pro 100 C4/W3-Professuren im Zeitverlauf

>> Anzahl der W3/C4-Professuren im Zeitverlauf

\section{Abbildung >> Aufenthalte von AvH-Geförderten im Zeitverlauf}

9.01

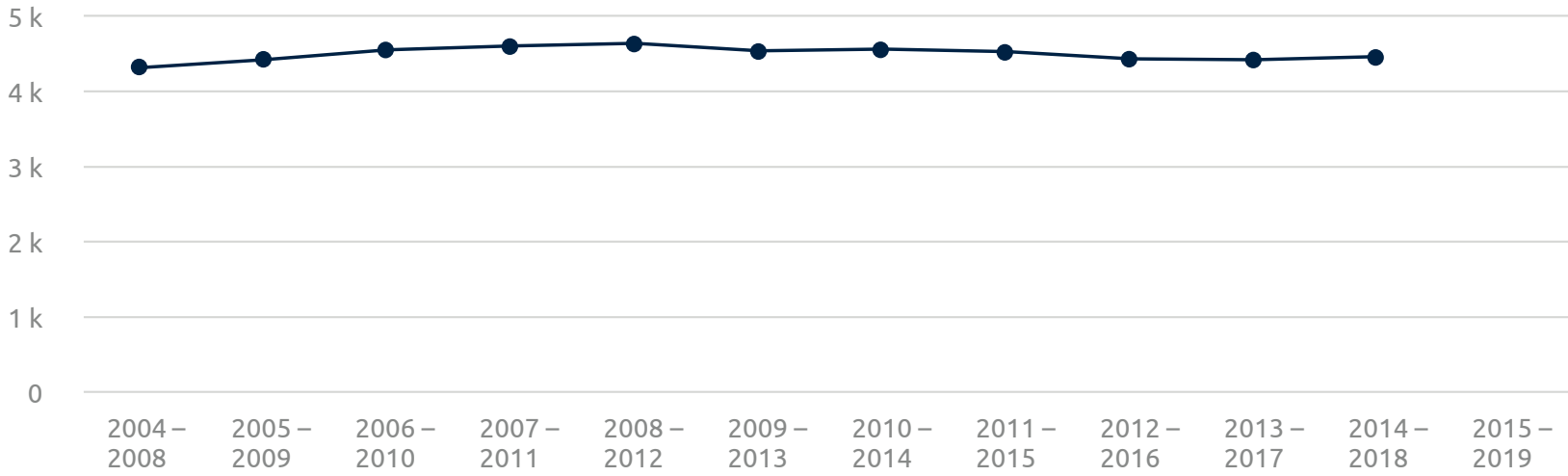


Abbildung > Aufenthalte von AvH-Geförderten im Zeitverlauf:

9.02 Große, mittelgroße und kleine Universitäten

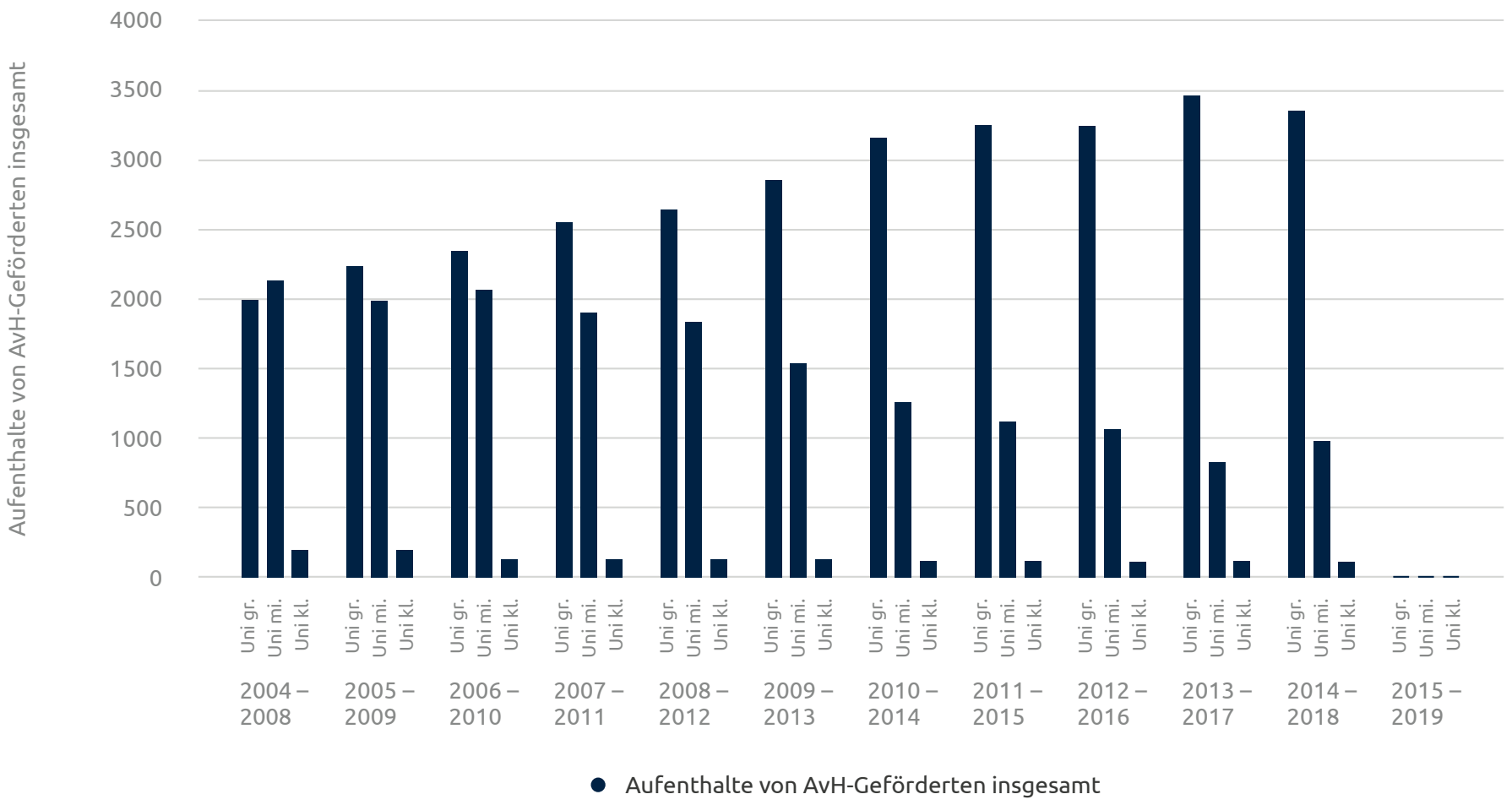

Abbildung >> Aufenthalte von AvH-Geförderten im Zeitverlauf:

9.03

Fachhochschulen

30

20

15

10

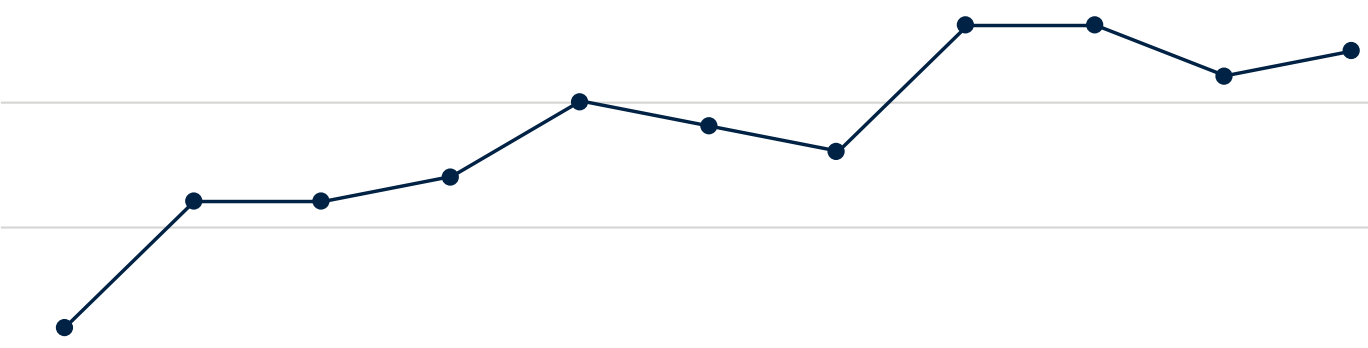

0

$\begin{array}{llllllllllll}2004- & 2005- & 2006- & 2007- & 2008- & 2009- & 2010- & 2011- & 2012- & 2013- & 2014- & 2015- \\ 2008 & 2009 & 2010 & 2011 & 2012 & 2013 & 2014 & 2015 & 2016 & 2017 & 2018 & 2019\end{array}$

- Aufenthalte von AvH-Geförderten insgesamt 
Im aktuellen Zeitraum konzentrieren sich über 95\% der Aufenthalte von AvH-Geförderten auf die großen und mittelgroßen Universitäten. Während die Verteilung zwischen diesen beiden Clustern bei der Fünfjahreskohorte 2004-2008 noch annähernd gleichhoch war (1.996 Aufenthalte an großen Universitäten gegenüber 2.143 Aufenthalte an mittelgroßen Universitäten), hat sie sich im weiteren Verlauf zugunsten der großen Universitäten verschoben. Im Fünfjahreszeitraum 2014-2018 entfielen 3.365 bzw. 74,9\% aller Forschungsaufenthalte ausländischer Wissenschaftlerinnen und Wissenschaftler auf die großen Universitäten und nur noch 978 bzw. 21,8\% auf die mittelgroßen. Auch die Zahl sowie der Anteil der Aufenthalte an kleinen Universitäten ist gesunken: von 196 bzw. 4,5\% aller Aufenthalte im Fünfjahreszeitraum 2004-2008 auf 110 bzw. 2,4\% im Zeitraum 2014-2018. Abb. 9.02
Fachhochschulen sind nur selten Ziel ausländischer Humboldt-Gastwissenschaftlerinnen und -wissenschaftler; im Fünfjahreszeitraum 2014-2018 wurden nur insgesamt 27 Aufenthalte von AvH-Geförderten verzeichnet. Trotz der kleinen Zahlen zeigt sich dennoch ein steigender Trend: in den Jahren 2004-2008 waren es nur 16 Aufenthalte. Abb. 9.03

Der relative Anteil der Aufenthalte von AvH-Stipendiatinnen und -Stipendiaten sowie -Preisträgerinnen und -trägern gemessen an der Zahl der C4/W3-Professuren ermöglicht eine Betrachtung unter Berücksichtigung der Größe bzw. Ausstattung der Hochschule. Die Zahl der Aufenthalte pro 100 C4/W3-Professuren unterscheidet sich deutlich zwischen den Hochschulclustern. Mit zuletzt rund 42 AvH-Geförderten pro 100 Professuren sind große Universitäten besonders attraktiv für ausländische Wissenschaftlerinnen und Wissenschaftler, vor den mittelgroßen Universitäten $(21,4)$ und den kleinen Universitäten $(10,3)$. Abb. $\mathbf{9 . 0 4}$

\section{Abbildung > Aufenthalte von AvH-Geförderten pro 100 C4/W3-Professuren:

50

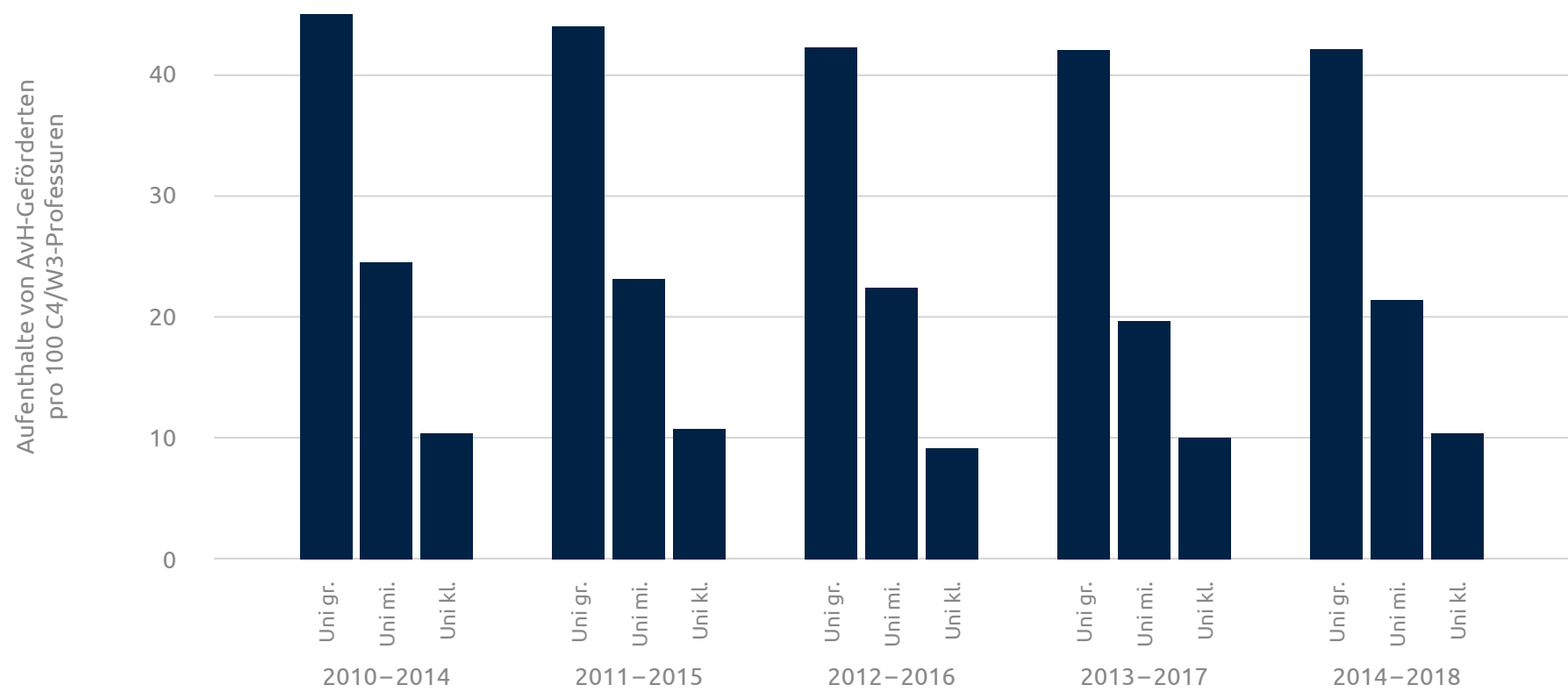

- Aufenthalte von AvH-Geförderten pro 100 C4/W3-Professuren 


\subsection{Verteilung der Aufenthalte von AvH-Geförderten nach Fächergruppen}

Im aktuellen Bericht erfolgt, in Angleichung an die anderen Themenbereiche, die Zuordnung der Aufenthalte von AvH-Stipendiatinnen und -Stipendiaten sowie -Preisträgerinnen und -trägern zu Fächergruppen auf der Basis der Systematik des Statistischen Bundesamtes (Destatis).

Gut die Hälfte (48\%) der Aufenthalte von AvH-Stipendiatinnen und -Stipendiaten sowie -Preisträgerinnen und -trägern entfiel im Fünfjahreszeitraum 2014-2018 auf die Fächergruppe Mathematik/Naturwissenschaften mit 2.158 Aufenthalten. Die Forschungsaufenthalte von Geförderten der Fächergruppe Geisteswissenschaften stellen etwa ein Viertel (26,3\%) aller Aufenthalte dar (1.180). Abb. 9.05

\section{Abbildung >> Aufenthalte von AvH-Geförderten nach Fächergruppe,

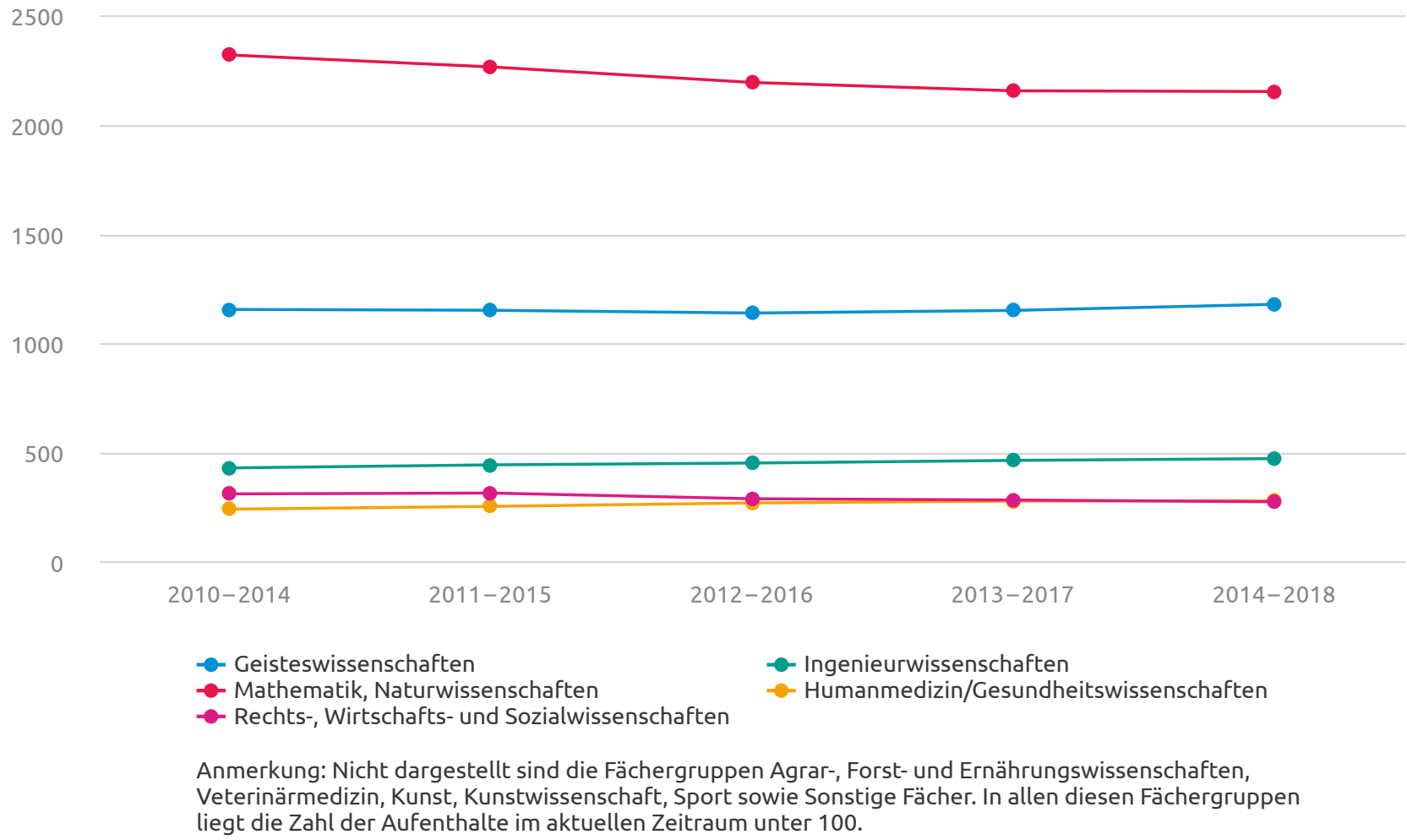

Siehe auch: >> Verteilung der Aufenthalte von AvH-Geförderten nach Fächergruppe

(in Prozent der Gesamtzahl der Aufenthalte) 


\subsection{Verteilung der Aufenthalte von AvH-Geförderten nach Herkunftsland}

Die Herkunft der AvH-Stipendiatinnen und -Stipendiaten sowie -Preisträgerinnen und -trägern lässt sich sowohl nach Land als auch nach Region auswerten. Die Abbildung 9.06 zeigt die Entwicklung der fünf wichtigsten Herkunftsländer der AvH-Geförderten in den letzten Jahren. Das wichtigste Herkunftsland sind die USA: im aktuellen Fünfjahreszeitraum 2014-2018 gab es aus diesem Land 717 Aufenthalte an deutschen Hochschulen. Auf dem zweiten Platz folgte mit deutlichem Abstand China mit 429 Aufenthalten, vor Indien mit 286 Aufent- halten. Mit Blick auf Europa weisen Stipendiatinnen und Stipendiaten sowie Preisträgerinnen und -träger aus Großbritannien die meisten Forschungsaufenthalte in Deutschland auf und dies mit einem klaren Aufwärtstrend: Zwischen dem Fünfjahreszeitraum 2010-2014 und dem Fünfjahreszeitraum 2014-2018 ist der Anteil der Aufenthalte von AvH-Geförderten aus Großbritannien an allen Aufenthalten von 4,6\% auf 5,5\% gestiegen. Die Zahl der AvH-Geförderten aus den USA ist im gleichen Zeitraum von 17,6\% auf 16,0\% gefallen. A Abb. 9.06

\section{Abbildung >> Aufenthalte von AvH-Geförderten nach Herkunftsland:

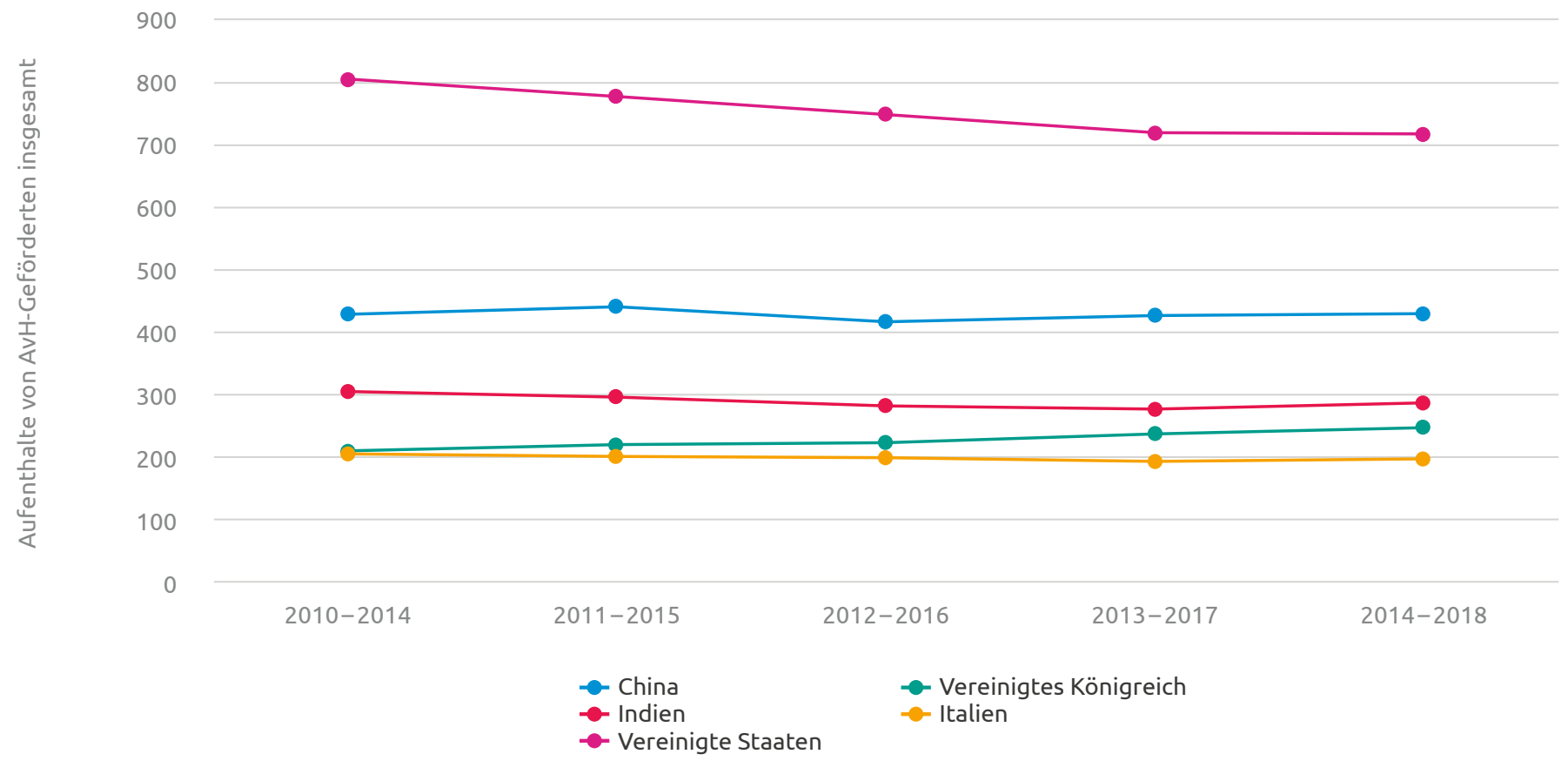

Siehe auch: >> Verteilung der Aufenthalte von AvH-Geförderten nach Herkunftsland: Top 5 Herkunftsländer, in Fünfjahreskohorten, 2014-2018 


\section{DFG-Projekte an Hochschulen}

- Der Anteil der internationalen DFG-Projekte liegt stabil bei etwa 13\%.

- Am häufigsten wurden 2019 Projekte mit Partnern in den USA (18,4\%), Frankreich $(16,6 \%)$ und Großbritannien $(11,1 \%)$ durchgeführt. Sichtbar zugenommen hat der Anteil der Projekte mit Kooperationspartnern an Forschungseinrichtungen in China (2018: 7,9\%; 2019: 9,5\%)

- Die fachliche Verteilung der DFG-Projekte an Hochschulen mit Partnern im Ausland ist über die Zeit weitgehend unverändert. 


\section{DFG-Projekte an Hochschulen}

Die Unterstützung internationaler Zusammenarbeit in der Forschung ist eine der satzungsgemäßen Aufgaben der Deutschen Forschungsgemeinschaft (DFG). Sie fördert internationale Kooperation und Austausch mit spezifischen Förderinstrumenten, unter anderem mit Forschungsstipendien und der Förderung im Programmbereich „Internationale wissenschaftliche Kontakte". Ein großer Anteil der Projekte mit internationaler Beteiligung geht dabei auf gemeinsame Ausschreibungen mit Partnerorganisationen im Ausland zurück. Dabei handelt es sich um Vereinbarungen der DFG mit ausländischen Partnerorganisationen zur gemeinsamen Begutachtung und Förderung internationaler Forschungsvorhaben. Im Jahr 2019 war sie insgesamt an 20 bi- und multilateralen Ausschreibungen beteiligt. Generell gilt, dass alle Förderverfahren der DFG für internationale Kooperationen offenstehen. Die Beteiligung internationale Kooperationspartner an Drittmittel-Projekten ist ein wichtiger Indikator für internationale Zusammenarbeit in der Forschung. Die hier aufbereiteten Kennzahlen zur Beteiligung internationaler Partner an DFG-Projekten an Hochschulen ergänzen andere forschungsrelevante Kennzahlen zu internationalen Publikationen, zur Förderung durch Drittmittel der EU, durch die AvH und den DAAD.

\section{Lesen Sie mehr zum Thema unter: >> HSI-Monitor > Themen > DFG-Projekte an Hochschulen}

\subsection{Grunddaten}

Im Jahr 2019 förderte die DFG 31.150 Projekte, rund 24.800 davon wurden an Hochschulen durchgeführt.

\section{Im Detail:}

>> DFG-Projekte an Hochschulen im Zeitverlauf

Gut 3.200 dieser 24.800 Projekte sehen eine Kooperation mit Partnern an Forschungseinrichtung im Ausland vor. Das sind etwa 100 Projekte mehr als im Vorjahr. Der Anteil der DFG-Projekte mit Partnern im Ausland an der Gesamtzahl der an Hochschulen geförderten Projekte liegt damit 2019 ähnlich wie im Vorjahr bei etwa 13\% (2018:12,7\%; 2019: 13,1\%). A Abb. 10.01

Der Anteil der Projekte mit Partnern im Ausland an der Gesamtzahl der geförderten Projekte unterscheidet sich zwischen den Universitätsclustern vergleichsweise wenig. Den höchsten Anteil an internationalen Projekten wies 2019 mit 14,0\% das Cluster der mittelgroßen Universitäten auf, gefolgt vom Cluster der großen Universitäten mit 12,7\% und dem Cluster der kleinen Universitäten mit 11,4\%. Abb. 10.02, S. 76

\section{Abbildung 10.01 \\ >> Anteil der DFG-Projekte an Hochschulen mit Partnern im Ausland an allen DFG-Projekten an Hochschulen im Zeitverlauf}

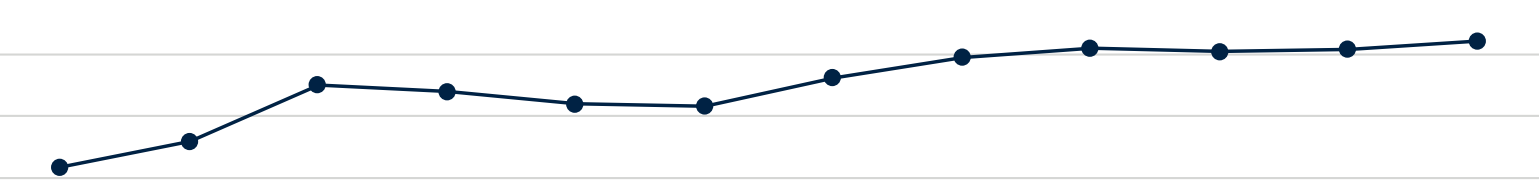


Abbildung > Anteil der DFG-Projekte an Hochschulen mit Partnern im Ausland an allen DFG-Projekten 10.02 an Hochschulen: Große, mittlere und kleine Universitäten, 2019

$15 \%$

$12,5 \%$

$10 \%$

$7,5 \%$

$5 \%$

$2,5 \%$

$0 \%$

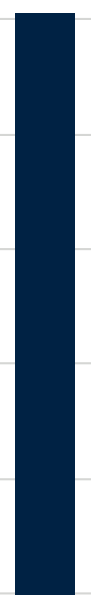

荘

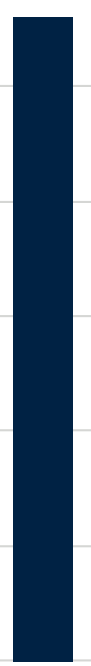

高

2019

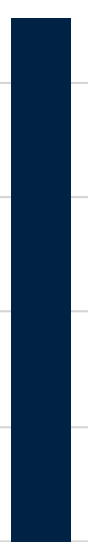

$\dot{\dot{\vec{c}}}$

- Anteil der DFG-Projekte an Hochschulen mit Partnern im Ausland an allen DFG-Projekten an Hochschulen

Abbildung >> Verteilung der DFG-Projekte an Hochschulen mit Partnern im Ausland 10.03 nach Fächergruppe, 2015-2019

$60 \%$

$50 \%$

$40 \%$

$30 \%$

$20 \%$

$10 \%$

$0 \%$

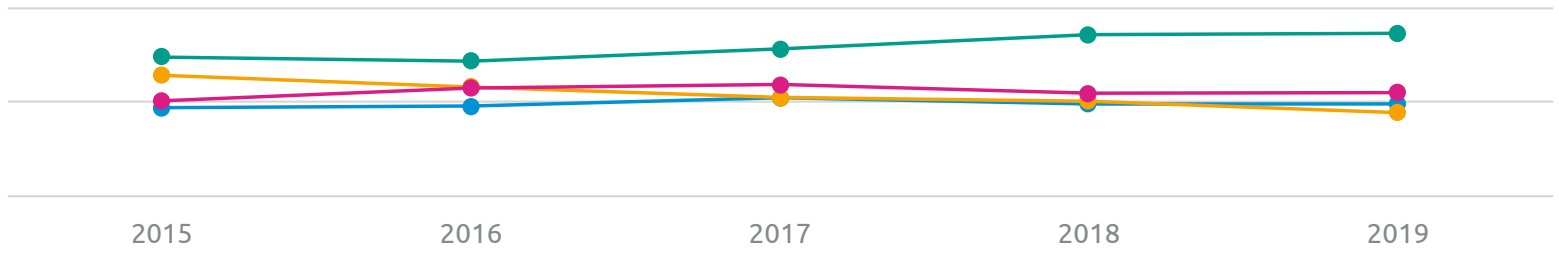

- Geisteswissenschaften

- Mathematik, Naturwissenschaften

- Rechts-, Wirtschafts- und Sozialwissenschaften
- Ingenieurwissenschaften

- Humanmedizin/Gesundheitswissenschaften
Anmerkung: Darstellung ohne die Fächergruppen „Sport”, „Kunst/Kunstwissenschaft” „Agrar-, Forst- und Ernährungswissenschaft" und „Sonstige Fächer", auf die im Berichtsjahr weniger als $1 \%$ entfallen.

Siehe auch: >> DFG-Projekte an Hochschulen mit Partnern im Ausland nach Fächergruppe, 2015-2019 


\subsection{Verteilung der DFG-Projekte an Hochschulen nach Fächergruppe}

Im aktuellen Bericht erfolgt, in Angleichung an die anderen Themenbereiche, die Zuordnung der DFG-Projekte an Hochschulen zu Fächergruppen auf der Basis der Systematik des Statistischen Bundesamtes (Destatis).

Die fachliche Verteilung der DFG-Projekte mit Partnern im Ausland ist über die Zeit betrachtet weitgehend unverändert. Im Jahr 2019 war knapp die Hälfte (49,6\%) aller DFG-Hochschul-Projekte mit Partnern im Ausland in der Fächergruppe Mathematik/Naturwissenschaften angesiedelt, gefolgt von Projekten mit Bezug zu den Ingenieurwissenschaften (17,2\%) und zu den Rechts-, Wirtschafts- und Sozialwissenschaften (10,9\%).

Leicht rückläufig sind die Anteile der Projekte in der Fächergruppe Humanmedizin/Gesundheitswissenschaften (2015: 12,8\%, 2019: 8,8\%). Der Anteil der Projekte in der Fächergruppe Ingenieurwissenschaften hat demgegenüber leicht zugenommen (2015: 14,7\%, 2019: 17,2\%). A Abb. 10.03

\subsection{Verteilung der DFG-Projekte an Hochschulen nach Partnerland}

Im Jahr 2019 waren Partner aus 114 Ländern an DFG-Projekten beteiligt. Auf die in der Abbildung dargestellten Länder entfallen zwei Drittel aller Kooperationen. Am häufigsten wurden Projekte mit Partnern in den USA (18,4\%), Frankreich (16,6\%), Großbritannien $(11,1 \%)$ und der Schweiz $(10,7 \%)$ durchgeführt. Sichtbar zugenommen hat die Zahl der gemeinsam mit Partnern an Forschungseinrichtungen in China durchgeführten Projekte (2018: 7,9\%, 2019: 9,5\%). Im Vergleich zum Vorjahr leicht abgenommen hat der Anteil der Projekte mit Kooperationspartnern in den USA (2018: 20,4\%; 2019: 18,4\%). Abb. 10.04

\section{Abbildung > Verteilung der DFG-Projekte an Hochschulen mit Partnern im Ausland 10.04 nach Partnerland, 2015-2019}

$25 \%$

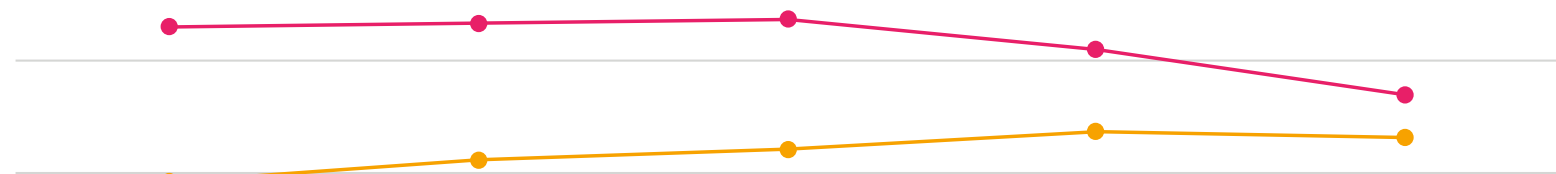

$15 \%$

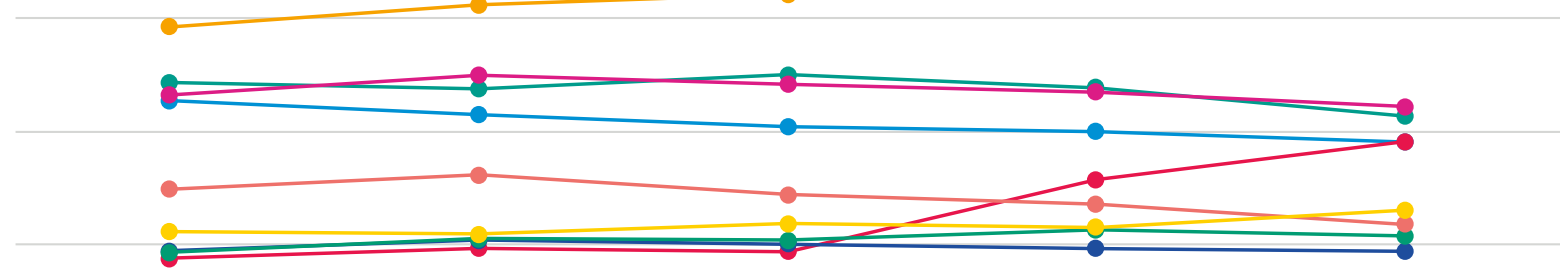

$0 \%$ 


\section{(- Drittmittel der Europäischen Union}

- Die deutschen Hochschulen haben 2018 knapp 700 Mio. Euro an Drittmitteln aus EU-Quellen eingeworben, das sind 19\% mehr als im Vorjahr.

- Der Anteil der EU-Drittmittel an allen Drittmitteln ist bei den kleinen Fachhochschulen mit 22,5\% sehr viel höher als bei anderen Hochschultypen.

- Der Betrag der von den Hochschulen aus dem Horizont 2020-Programm eingeworbenen Fördermitteln liegt seit 2016 zwischen 460 Mio. und 490 Mio. Euro. Über zwei Drittel der Einnahmen entfallen auf ERC-Grants und Fördermittel für Verbundforschung. 


\section{Drittmittel der Europäischen Union}

Die Einwerbung von Drittmitteln ist für Hochschulen ein wichtiger Faktor zur Finanzierung der Forschung geworden. Die Akquise von Drittmitteln europäischer Institutionen kann dabei als Indikator für die internationale Ausrichtung von Hochschulen dienen - zum einen, weil Mittel von internationalen Quellen eingeworben werden, und zum anderen, da die Anträge in der Regel eine internationale Dimension wie zum Beispiel die Beteiligung von Hochschulen und Akteuren aus mehreren Ländern aufweisen müssen.

\subsection{Grunddaten}

2018 haben deutsche Hochschulen etwa 695 Mio. Euro an Drittmitteln aus EU-Quellen eingeworben; das bedeutet einen Zuwachs von 19\% im Vergleich zum Vorjahr. Hiervon entfielen 91\% auf Universitäten und nur 8,8\% auf Fachhochschulen.

\section{Im Detail:}

\section{>> EU-Drittmittel insgesamt im Zeitverlauf}

Im Durchschnitt aller Hochschulen entsprachen die eingeworbenen EU-Drittmittel etwa 14.613 Euro pro Professur. Bei der Betrachtung der Hochschulcluster fallen jedoch starke Unterschiede auf: Bei den großen
Lesen Sie mehr zum Thema unter: >> HSI-Monitor > Themen > Drittmittel der Europäischen Union
Universitäten wurden 28.058 Euro pro Professur eingeworben, während große Fachhochschulen nur auf 2.347 Euro pro Professur und Kunst- und Musikhochschulen nur auf 517 Euro pro Professur kamen.

- Abb. 11.01

Auch innerhalb der Cluster ist die Bandbreite hoch und reicht von Hochschulen mit großen Drittmittelbeträgen bis hin zu einzelnen Hochschulen ohne eingeworbene Mittel. Vor allem bei den kleinen Fachhochschulen und den Kunst- und Musikhochschulen haben die meisten Hochschulen gar keine Mittel eingeworben. Dies erklärt auch die geringen Werte innerhalb dieses Clusters.

\section{Abbildung >> EU-Drittmittel pro Professur nach Cluster, 2018}

11.01

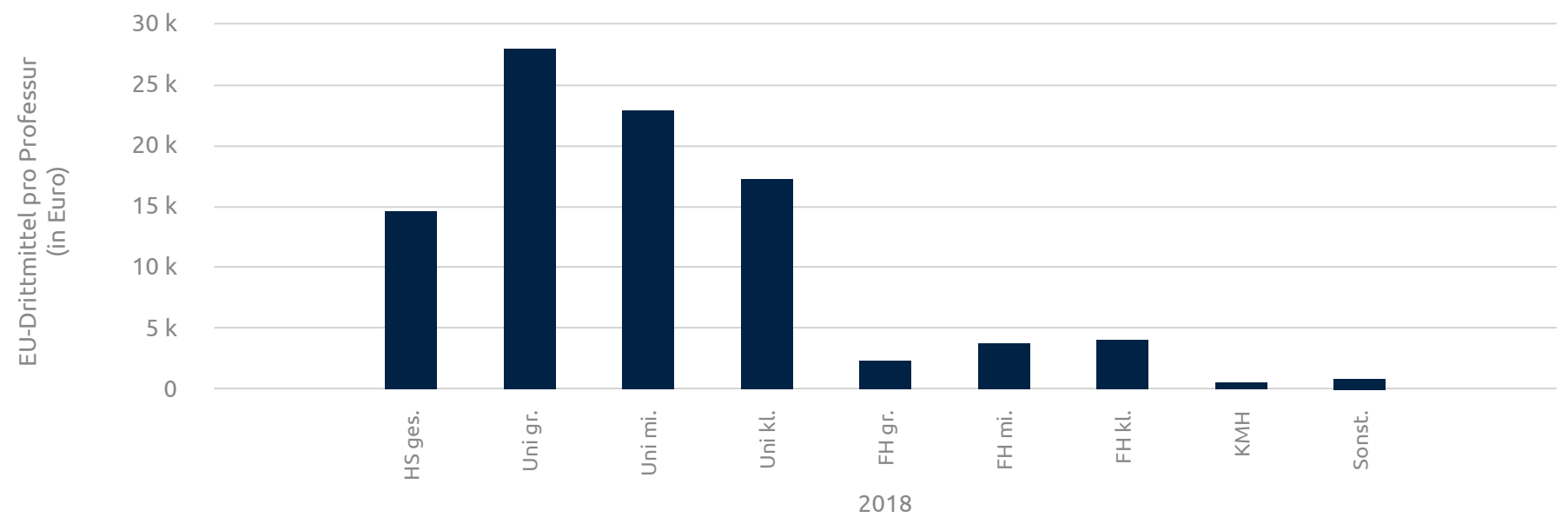

- EU-Drittmittel pro Professur (in Euro) 
Abbildung >> Anteil der EU-Drittmittel an allen Drittmitteln nach Cluster, 2018 11.02

$25 \%$

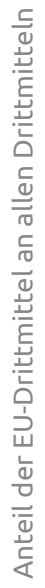

$20 \%$

$15 \%$

$10 \%$

$5 \%$

$0 \%$
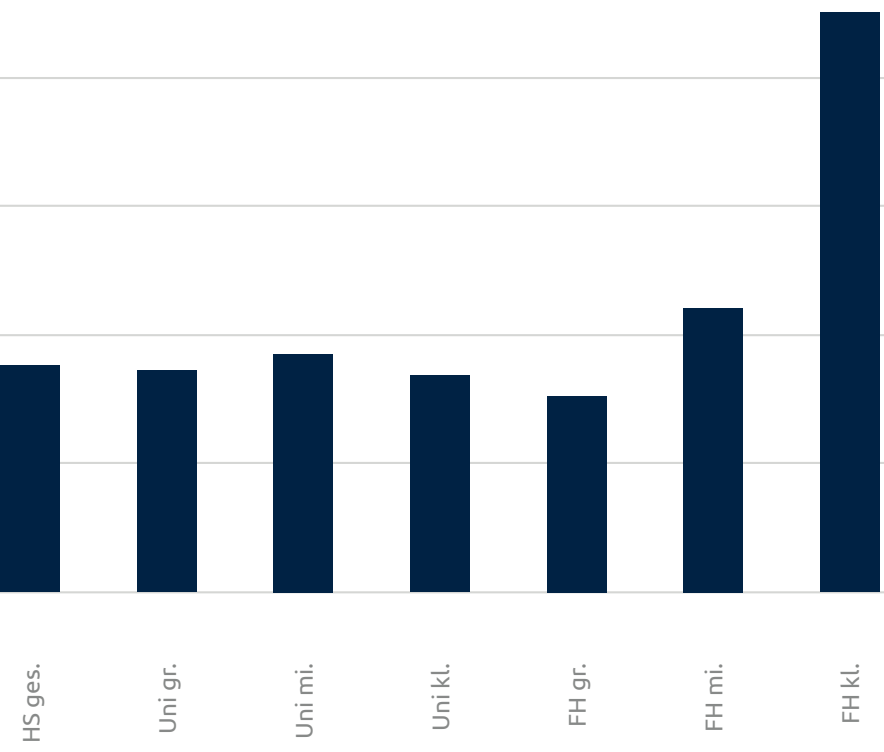

$\begin{array}{ll}\cdot \dot{\bar{E}} & \dot{\vec{x}} \\ \cdot \overline{\mathrm{C}} & \dot{\mathrm{S}}\end{array}$

俩

$\dot{E}$
I

$\frac{\dot{x}}{\text { I }}$

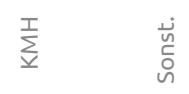

2018

- Anteil der EU-Drittmittel an allen Drittmitteln

Abbildung >> Horizont 2020-Fördermittel insgesamt im Zeitverlauf

11.03

$600 \mathrm{M}$

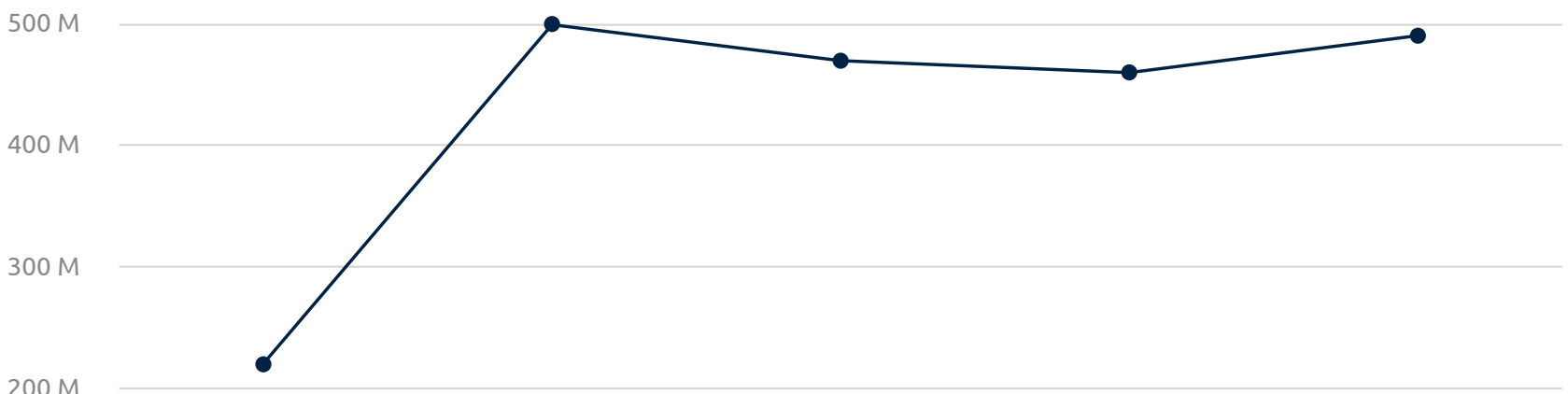

$100 \mathrm{M}$

0 
Bezogen auf die Anzahl der Professuren ist der Gesamtbetrag der EU-Drittmittel bei den Fachhochschulen zwar erheblich geringer als bei den Universitäten, die EU-Drittmittel stellen bei den Fachhochschulen jedoch einen deutlich höheren Anteil am Drittmittelbudget. Besonders hoch ist dieser Anteil bei den kleinen Fachhochschulen (2018: 22,5\% aller Drittmittel). Bei mittelgroßen Fachhochschulen und mittelgroßen Universitäten liegt der Anteil der EU-Drittmittel am gesamten Drittmittelbudget bei 11,0\% bzw. 9,3\%. Durchschnittlich liegt der Anteil der EU Drittmittel an allen Drittmitteln an deutschen Hochschulen bei 8,8\%. Abb. 11.02
Bis auf die großen Fachhochschulen, bei denen der Anteil der EU-Drittmittel an allen Drittmitteln seit 2014 stark rückläufig ist, hat dieser bei allen deutschen Hochschulen von 2017 auf 2018 zugenommen.

\section{Im Detail:}

> Anteil EU-Drittmittel an allen Drittmitteln im Zeitverlauf: Große Fachhochschulen

\subsection{Fördermittel aus dem EU-Forschungsprogramm Horizont 2020}

Einen besonders wichtigen Teil der EU-Drittmittel stellen die Fördermittel aus „Horizont 2020", dem achten Rahmenprogramm der Europäischen Union für Forschung und Innovation, dar. Hierauf können sich neben Universitäten und Hochschulen auch Forschungsinstitute und Unternehmen bewerben. Daten zu den von den Hochschulen eingeworbenen Fördermitteln sind seit 2015 verfügbar. ${ }^{10}$

Nach einem raschen Anstieg der von den Hochschulen eingeworbenen Mittel von 219 Mio. Euro im Jahr 2015 auf 499 Mio. Euro im Jahr 2016, lag der Gesamtbetrag seitdem zwischen 460 und 490 Mio. Euro. ${ }^{11}$ Abb. 11.03
97\% der Horizont 2020-Fördermittel entfielen im Jahr 2019 auf die Universitäten, davon rund zwei Drittel $(67,7 \%)$ allein auf die großen Universitäten. Etwa ein Viertel (25,3\%) aller Fördermittel aus diesem-Programm wurden von mittelgroßen Universitäten eingeworben, während Fachhochschulen nur 2,4\% erhielten.

Die mehr als 40 Programmlinien von Horizont 2020 werden im HSI-Monitor in Anlehnung an eine Systematik des DLR-Projektträgers für Horizont 2020 (EU-Büro des BMBF) in fünf Kategorien zusammengefasst.

Die durchschnittliche Verteilung der Mittel zwischen den fünf Kategorien war zwischen 2017 und 2019 wie folgt. Die einzelnen Zeitreihen sind im HSI-Monitor verfügbar.

\begin{tabular}{|c|c|c|c|c|c|c|c|}
\hline Kategorie & 2015 & 2016 & 2017 & 2018 & 2019 & \multicolumn{2}{|c|}{ Zeitreihen im HSI-Monitor } \\
\hline ERC Grants & $35,9 \%$ & $39,7 \%$ & $44,5 \%$ & $45,6 \%$ & $43,9 \%$ & >> Gesamtbetrag & >> Anteil an H2020 \\
\hline Euratom & $10,0 \%$ & $1,0 \%$ & $0,0 \%$ & $1,7 \%$ & $0,0 \%$ & >> Gesamtbetrag & >> Anteil an H2020 \\
\hline $\begin{array}{l}\text { Marie-Sklodowska-Curie } \\
\text { Actions (MSCA) }\end{array}$ & $21,2 \%$ & $10,0 \%$ & $11,5 \%$ & $14,8 \%$ & $10,9 \%$ & >> Gesamtbetrag & >> Anteil an H2020 \\
\hline Verbundforschung & $29,7 \%$ & $45,1 \%$ & $36,0 \%$ & $35,7 \%$ & $35,2 \%$ & >> Gesamtbetrag & >> Anteil an H2020 \\
\hline Sonstige Förderlinien & $3,1 \%$ & $4,3 \%$ & $8,0 \%$ & $2,2 \%$ & $10,1 \%$ & >> Gesamtbetrag & > Anteil an H2020 \\
\hline
\end{tabular}

10 Daten zu den prestigeträchtigen ERC-Grants sind bereits ab 2009 verfügbar. Ab 2015 werden die ERC-Grants als Teil von Horizont 2020 behandelt.

11 Zu beachten ist, dass bei den Förderungen aus dem Horizont 2020 Programm die Gesamtzuwendungen unabhängig von der Dauer der Projekte dem Jahr des Vertragsabschlusses zugeordnet werden. Diese ungleiche Verteilung bei mehriährigen Förderungen kann sowohl bei der Gesamtsumme und speziell auch bei den Teilprogrammen sowie bei der Auswertung nach Hochschulcluster zu signifikanten jährlichen Schwankungen führen. 


\section{Über das Projektteam}

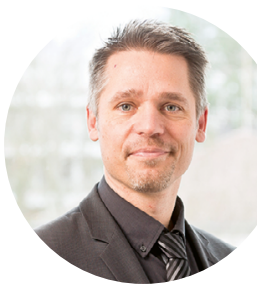

\section{Axel Karpenstein}

DAAD

Leitung der Gemeinsamen

Geschäftsstelle

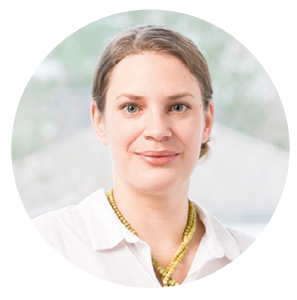

\section{Diandra Dachtera}

DAAD

Koordination der

Gemeinsamen Geschäftsstelle

Tel: (0228) 882-8983

Tel: (0228) 882-8794

kontakt@hsi-monitor.de

www.daad.de

kontakt@hsi-monitor.de

www.daad.de

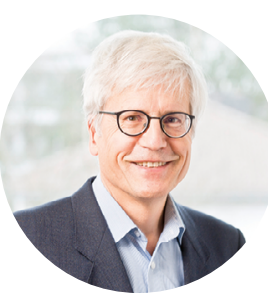

\section{Dr. Christian Schäfer}

DAAD

Projektleitung DAAD

www.daad.de

\section{Marijke Wahlers}

HRK

Projektleitung HRK

www.hrk.de 

Der Deutsche Akademische Austauschdienst (DAAD) ist die Organisation der deutschen Hochschulen und ihrer Studierenden zur Internationalisierung des Wissenschaftssystems. Er schafft Zugänge zu den besten Studien- und Forschungsmöglichkeiten für Studierende, Forschende und Lehrende durch die Vergabe von Stipendien, fördert transnationale Kooperationen und Partnerschaften zwischen Hochschulen und ist die Nationale Agentur für die europäische Hochschulzusammenarbeit. Der DAAD unterstützt damit die Ziele der Auswärtigen Kultur- und Bildungspolitik, der nationalen Wissenschaftspolitik und der Entwicklungszusammenarbeit. Dafür unterhält ein Netzwerk mit 69 Auslandsbüros und rund 426 Lektoraten weltweit sowie die internationale DAAD-Akademie (iDA) Der DAAD wird überwiegend aus Bundesmitteln des Auswärtigen Amts, des Bundesministeriums für Bildung und Forschung, des Bundesministeriums für wirtschaftliche Zusammenarbeit und Entwicklung und der Europäischen Union finanziert.

Die Deutsche Forschungsgemeinschaft (DFG) ist die zentrale Selbstverwaltungsorganisation der Wissenschaft in Deutschland. Organisiert ist die DFG als privatrechtlicher Verein. Ihre Mitglieder sind forschungsintensive Hochschulen, außeruniversitäre Forschungseinrichtungen, wissenschaftliche Verbände sowie die Akademien der Wissenschaften. Die Kernaufgabe der DFG besteht in der wettbewerblichen Auswahl und Finanzierung von Forschungsvorhaben an Hochschulen und Forschungsinstituten. Hierfür steht der DFG jährlich ein Budget von mehr als 3 Milliarden Euro zur Verfügung, das sie überwiegend von Bund und Ländern, aber auch aus EU-Mitteln und privaten Zuwendungen erhält.

\section{HRK Hochschulrektorenkonferenz} Die Stimme der Hochschulen

Die Hochschulrektorenkonferenz (HRK) ist der Zusammenschluss der staatlichen und staatlich anerkannten Hochschulen in Deutschland. Sie hat gegenwärtig 268 Mitgliedshochschulen, in denen über 92 Prozent aller Studierenden in Deutschland immatrikuliert sind. Die HRK ist die Stimme der Hochschulen gegenüber Politik und Öffentlichkeit und das zentrale Forum für ihren gemeinsamen Meinungsbildungsprozess. Die HRK kooperiert mit Hochschulen und Partnerorganisationen weltweit. Sie vertritt dabei die Interessen der deutschen Hochschulen auf internationaler Ebene und unterstützt ihre Mitglieder im Internationalisierungsprozess.

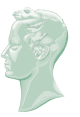

\section{Alexander von Humboldt} Stiftung/Foundation

Die Alexander von Humboldt-Stiftung fördert Wissenschaftskooperationen zwischen exzellenten ausländischen und deutschen Forscherinnen und Forschern. Sie vergibt jährlich über 700 Stipendien und Preise, mit denen Wissenschaftlerinnen und Wissenschaftler aus dem Ausland nach Deutschland kommen, um ein selbst gewähltes Forschungsprojekt mit einer Gastgeberin oder einem Gastgeber durchzuführen. Wissenschaftlerinnen und Wissenschaftler aus Deutschland können mit Unterstützung der Stiftung ins Ausland gehen und dort mit einem Mitglied des Humboldt-Netzwerks zusammenarbeiten. In weltweit über 140 Ländern pflegt die Stiftung ein fächerübergreifendes Netzwerk von mehr als 30.000 Humboldtianerinnen und Humboldtianern unter ihnen 56 mit Nobelpreis. 